\title{
1 Energy transformation on flow-induced motions of 2 multiple cylindrical structures with various corner 3 shapes
}

4 Yibo Liang (梁艺博) ${ }^{1}$, Longbin Tao ${ }^{1,}$, , Longfei Xiao (肖龙飞) $)^{2,3}$

$5{ }^{1}$ Department of Naval Architecture, Ocean and Marine Engineering, University of

6 Strathclyde, Glasgow, G4 OLZ, UK

$7 \quad{ }^{2}$ State Key Laboratory of Ocean Engineering, Shanghai Jiao Tong University, Shanghai, 8 200240, China

$9{ }^{3}$ Collaborative Innovation Centre for Advanced Ship and Deep-Sea Exploration, 10 Shanghai, 20040, China

11 Abstract draft semi-submersible (DDS), a typical multiple cylindrical structure in offshore engineering was carried out to investigate the energy transformation of the vortex shedding process. In addition, the corner shape effect on the flow characteristics, the hydrodynamic forces and the FIM responses are presented for a multiple cylindrical structure with various corner shapes (sharp, rounded and chamfered) under $45^{\circ}$ current incidence. Different energy transformations, hydrodynamic characteristics and FIM responses were observed due to the slight variation of the corner shape. The galloping at $45^{\circ}$ incidence for a square-section shape column was observed when the corner shape modified as a chamfered corner. A "re-attached vortex shedding" phenomenon is

\footnotetext{
* Corresponding author. Tel: +44 (0)141 5483315

Email address: longbin.tao@strath.ac.uk
} 
discovered when the "lock-in" happened for a chamfered corner design. Further insights of the fluid physics on the flow characteristics due to the difference of the corner shape are revealed. In addition, the energy transformation and the mechanism for reducing the hydrodynamic forces and the FIM responses are analysed.

\section{Keywords}

Flow-induced motions (FIM); Vortex-induced motions (VIM); Corner shapes; Vortex shedding; Energy transformation; Galloping

\section{Introduction}

Flow-induced motions (FIM) introduced a class of flows exhibiting a coupled interaction between fluid and structure. For example, vortex-induced motions (VIM) and galloping are some of this type of phenomenon. FIM attracts strong research interest in the field of fluid and structure interaction. Both VIM and galloping have received considerable attention in the offshore engineering discipline. Hydrodynamic problems of FIM with bluff column are often encountered during the operations of offshore platforms. Since the Genesis Spar commissioned in $1997^{1,2}$, vortex-induced motion (VIM) - a cyclic rigid body motion induced by vortex shedding has been regularly observed on large floating offshore structures ${ }^{3,4}$ (e.g., Spar, semi-submersible and tension-leg platform) due to the long-term strong loop current in the Gulf of Mexico (GoM). Fujarra, et al. ${ }^{2}$ well documented the literature about VIM during the last decade.

When a current flow past an offshore platform, the vortices in the wake region can generate strong cyclic dynamic effects on the platform which is known as VIM. The VIM is mainly characterized as the motion in the horizontal plane leading to potential 
damage particularly causing the fatigue to mooring and riser systems. Apart from VIM, the FIM phenomenon of galloping is worth to investigate as well. Studies focusing on a typical square section cylinder with a flat face normal to the flow have been carried out in the aerodynamic discipline since Den $\operatorname{Hartog}^{5}$ first proposed a criterion for the onset of galloping. However, there is still lack of understanding on the galloping in the hydrodynamic side.

Most of the floating platforms consist serval columns to support the superstructure. For a multi-column offshore platform (e.g. semi-submersible, tension-leg platform), vortex shedding occurs around each column. A strong vortex shedding interaction can be observed between each single column. Investigations on these interactions have been carried out by many researchers. Liu and Jaiman ${ }^{6}$ performed a numerical study of vortexinduced vibrations (VIV) in a side-by-side cylinder arrangement. Li, et al. ${ }^{7}$ further investigated the coupled dynamics of VIV adjacent to a stationary wall. Recently, a stability analysis of the flow-induced vibrations (FIV) of two cylinders in tandem arrangement was provided by Yao and Jaiman ${ }^{8}$. Even with considerable research effort on FIV, most of the current studies are still focusing on the low Reynolds number $(\operatorname{Re} \approx$ 100) problem. Literatures on the FIV study at low Reynolds numbers have been published by different researchers focusing in various areas (e.g. Shen and $\mathrm{Sun}^{9}$, Zhu, et al. ${ }^{10}$, Jiao and $\mathrm{Wu}^{11}$ ), and most of them are expected to reveal more insight on the physics under high Reynolds number in future. To date, most of the study are still limited in the laminar flow problem. One of the contribution of the present work is that the Reynolds number in the current study reaches to the order of $10^{4}$, where turbulence plays an important role in the fluid-structure interaction during FIV. 
In addition, cylinders investigated in the previous studies are either with a $2 \mathrm{D}$ assumption or are of an infinite length. It is noted that most of the floating structures in the ocean are with finite length columns and some of them are connected by pontoons. Therefore, the free end effect need to be examined. Rastan, et al. ${ }^{12}$ recently performed a study on the flow around a single wall-mounted square cylinder at low Reynolds numbers. There are few papers contributing to the examination of the physics of FIV on a multi finite length column structure. Therefore, the second contribution of the present study is to provide a comprehensive numerical study to examine the mechanism of FIV on a multi finite length column structure based on our well-validated numerical model ${ }^{13,14}$.

Apart from the Reynolds number and the free end effect, the shape of the column, especially the corner shape, affects the hydrodynamic and FIM responses. The corner shape of the column can alter the vortex shedding characteristics around columns significantly. Bearman, et al. ${ }^{15}$ experimentally investigated the corner radius influence on the force experienced by a square or diamond section-shaped column in an oscillating flow. Their study showed that the drag coefficient of a diamond section decreases with increasing the corner radius. However, the square section does not show a clear relationship between drag coefficient and corner radius. Subsequently, $\mathrm{Hu}$, et al. ${ }^{16}$ experimentally studied the corner radius effects on a square prism based on the particle image velocimetry (PIV) measurement in the wake region. Liu, et al. ${ }^{17}$ recently carried out a numerical study about the corner radius effects on VIM of a semi-submersible, and reported that the transverse motion is significantly affected by the corner ratio of the column. Tamura, et al. ${ }^{18}$ performed a numerical study on flow over a square column with different corner shapes including sharp, rounded and chamfered. Both hydrodynamic force and pressure distribution were discussed in their study. Subsequently, Tamura and 
Miyagi ${ }^{19}$ implemented a wind tunnel test to obtain the static hydrodynamic forces (drag and lift forces) on the cylinder with various corner shapes, and the authors confirmed that the chamfered and rounded corners lead to decreased drag forces, as a result of a reduction in wake width. Recently, Cao and Tamura ${ }^{20}$ further performed a numerical study on supercritical flow past a square cylinder with rounded corners. However, the square cylinder itself is still a stationary structure without any motions coupled in the simulation. Despite the aforementioned efforts, there is still lack of comprehensive understanding of the corner shape effect, especially on the motions induced by the vortex shedding due to different corner shapes. The third contribution of the current work is to provide insights on the corner shape effect.

It is also worth noting that most research on a square cylinder focused on an angle of attack at 0 degree where FIM is dominated by galloping. At an angle of attack at 45 degree, however, VIM dominates FIM. Zhao, et al. ${ }^{21}$ defined the branch/mode competition in the flow-induced motions of a single square cylinder. The energy transformation between the fluid and the single cylinder are well examined in their experimental tests. Unlike most of the previous studies on FIM, the time-frequency domain is analysed by using continuous wavelet transforms (CWT) instead of Fast Fourier Transform (FFT). As a traditional way, FFT has been used by many researchers on studies of FIM, Zhao, et al. ${ }^{22}$ well-illustrated the flow pattern against oscillating amplitude, frequency and phase characteristics. Liu, et al. ${ }^{17}$ also tried to used frequency domain analysis to investigate FIM. Gonçalves, et al. ${ }^{23}$ applied Hilbert-Huang Transform (HHT) to examine the frequency characteristics of FIM. It is noted that Continuous Wavelet Transform (CWT) is very efficient in determining the damping ratio of oscillating signals (e.g. identification of damping in dynamical systems). CWT can 
115 illustrate the time history in the frequency domain. This new routine can provide more

116 information on the energy transformation between the fluid and oscillating structure

117 leading to a better understanding of FIM. Apart from analysing the energy transformation

118 on the frequency domain, the work done is a straight way to observe the energy

119 transformation process. Antony, et al. ${ }^{24}$ investigated the work done by each column of a

120 multi-column floating structure through experiments. Liang and $\mathrm{TaO}^{25}$ later performed a

121 numerical study on the work done by each column on a multiple cylindrical structure.

122 Apart from analysing the energy transformation in frequency domain, calculating the

123 work done by the structure is a straightforward way to observe the energy transformation

124 process. Antony, et al. ${ }^{24}$ investigated the work done by each column of a multi-column

125 floating structure through experiments. Liang and $\mathrm{Tao}^{13}$ later performed a numerical

126 study on the work done by each column on a multiple cylindrical structure.

Based on the literature, a comprehensive numerical investigation is performed in

128 the present study to reveal further insights of the fluid physics on the effects of corner

129 shape design on vortex shedding characteristics and associated VIM by examining the 130 energy transformation of the hydrodynamic phenomenon. Considerable studies are 131 provided in the present work to exam the energy transformation based on the continuous 132 wavelet transform (CWT). It is confirmed that the flow characteristics, hydrodynamic 133 forces and the related VIM responses altered dramatically by varying the corner shape. 134 Additionally, the galloping at $45^{\circ}$ incidence for a square-section shape column was 135 observed when the corner shape modified as a chamfered corner.

\section{Fundamental description of FIM phenomenon}

\subsection{Description of FIM}


As a typical cyclic rigid body motion, FIM is induced by vortex shedding from a

139 large-sized floating structure. When the current flow over a floating cylindrical

140 structure, the dynamics of the structure will be affected by the vortices that are

141 generated and then systematically shed in the downstream region, may begin oscillating

142 either in a side to side or in a fore and aft manner. If the vortex shedding frequency is

143 approaching to the natural frequency of the structure, a so-called "lock-in" phenomenon

144 can occur, which could amplify the cyclic motions of the structure dramatically. This

145 resonance phenomenon may lead to potential damage to offshore systems, especially

146 causing fatigue of the mooring and riser systems.

\subsection{Key parameters for FIM}

To better understand the phenomenon of FIM, primary non-dimensional parameters have been introduced into the present work. In this section, all the key nondimensional parameters are presented following the equations to give general information describing FIM.

The so-called reduced velocity $(U r)$ is normally used as the reference value

153 when discussing FIM, and is defined as:

$$
U r=\frac{U T_{n}}{D}
$$

155 where $U$ is the current speed, $T_{n}$ is the natural period of the structure motions in calm water and $D$ is the projected length of the column. 
159 frequency, $f_{v}$. A dimensionless variable named as Strouhal number $(S t)$ is often used to

160 represent the vortex shedding frequency, which is given by:

161

$$
S t=\frac{f_{v} D}{U}
$$

162 where $f_{v}$ is the vortex shedding frequency that is obtained from the power spectra of the 163 lift force fluctuations as suggested by $\operatorname{Schewe}^{26}, U$ is the free stream velocity and $D$ is

164 the projected width of the column. The Strouhal number for square cylinders, depending 165 on the current incidence, were shown to be 0.13 and 0.17 for $0^{\circ}$ and $45^{\circ}$ incidence

166 respectively. These results were obtained by Norberg ${ }^{27}$ from his experimental study.

167 As the vortex shedding is a flow separation phenomenon, the Reynolds number 168 is used to describe the level of the flow separation.

$$
\operatorname{Re}=\frac{U D}{v}
$$

where $U$ is the free stream velocity, $D$ is the projected width of the column and $v$ is the kinematic viscosity of the fresh water.

With Reynolds number increases, the flow characteristics around a cylinder will have different separation phenomena due to the viscous effects. The vortex shedding phenomenon can vary significantly by increasing the Reynolds number.

$$
C_{D}(t)=\frac{F_{D}(t)}{\frac{1}{2} \rho U^{2} A_{\text {projected }}}
$$




$$
C_{L}(t)=\frac{F_{L}(t)}{\frac{1}{2} \rho U^{2} A_{\text {projected }}}
$$

181 where, $F_{D}(t)$ is the drag force on the structure, $F_{L}(t)$ is the lift force on the structure, $\rho$ is

182 the density of the fresh water, $U$ is the free stream velocity and $A_{\text {projected }}$ is the projected 183 area. current on the structure are calculated by the equation ${ }^{28}$ :

$$
\begin{aligned}
& m \ddot{X}(t)+C \dot{X}(t)+K_{x} X(t)=F_{x}(t) \\
& m \ddot{Y}(t)+C \dot{Y}(t)+K_{y} X(t)=F_{y}(t)
\end{aligned}
$$

where $m$ is the platform mass; $C$ is the structural damping coefficient; $K_{x}$ and $K_{y}$ are the linear spring constant in the in-line and transverse directions; $X(t)$ and $Y(t)$ are the displacement at in-line and transverse direction, respectively; $F_{x}(t)$ and $F_{y}(t)$ represent the

191 in-line and transverse hydrodynamic forces acting on the structures.

The structural damping coefficient is very small and can be disregarded. The

193 hydrodynamic forces which include added mass and hydrodynamic damping forces due 194 to fluid are placed on the right side of the equations. amplitude $(A / D)$ is chosen as the common variable $2,3,23,29$, which is defined as:

197

$$
A / D=\sqrt{2} \times \sigma\left(\frac{y(t)}{D}\right)
$$


198 where $\sigma$ is the standard deviation of the time series $y(t) / D$, and $y(t)$ represents the time

199 series of in-line, transverse and yaw motions. For the rotational yaw motion, the non-

200 dimensional amplitude is defined as $\sqrt{2} \times \sigma(\operatorname{yaw}(t))$.

\section{Numerical simulation}

In the present study, the deep-draft semi-submersible consists of 4 columns. The vortices shed from each column will generate periodically hydrodynamic loads on the overall structure. Thus, the shapes of the columns and the subsequent interactions between the individual vortex shedding processes due to each column, characterize the VIM responses.

Fig. 1 shows an overview of the semi-submersible along with the chronological 208 order of the columns. In Table 1, the model characteristics of the semi-submersible 209 were illustrated. As shown in Fig. 2, four horizontal mooring lines are attached to 210 restrain the horizontal motions of the semi-submersible model. In the present numerical 211 model, the horizontal stiffness at both the transverse and in-line directions is $66.5 \mathrm{~N} / \mathrm{m}$

212 which was scaled from a prototype mooring design. In addition, only three degrees

213 freedom motions in the horizontal plane (namely transverse, in-line and yaw) were 214 allowed in the numerical simulations. 
A)

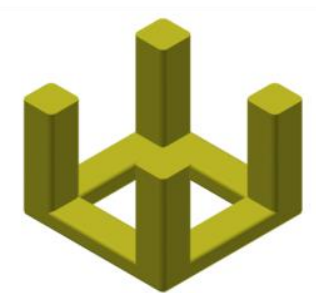

B)

215

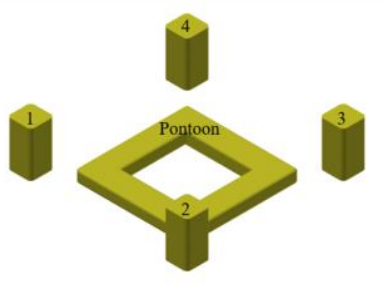
of 1:64).
C)

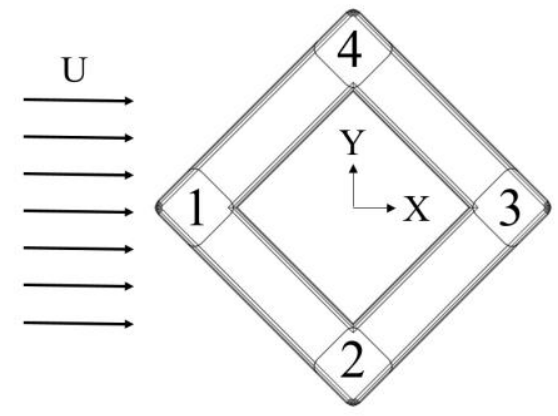

Fig. 1 Numerical model (rounded corner as an example) simulated in the present study ( $\mathrm{A}$ is the entire model; $\mathrm{B}$ is the decomposed model which shows the definition of the individual members; $\mathrm{C}$ is the sketch of the semi-submersible).

Table 1 Principle dimensions of the model semi-submersible (with a scale ratio

\begin{tabular}{ll}
\hline & Model $(\mathbf{m})$ \\
\hline Distance between centre columns $(\boldsymbol{S})$ & 1.133 \\
Column width $(\boldsymbol{L})$ & 0.305 \\
Immersed column height above the pontoon $(\boldsymbol{H})$ & 0.578 \\
Pontoon height $(\boldsymbol{P})$ & 0.156
\end{tabular}




\section{Transverse}

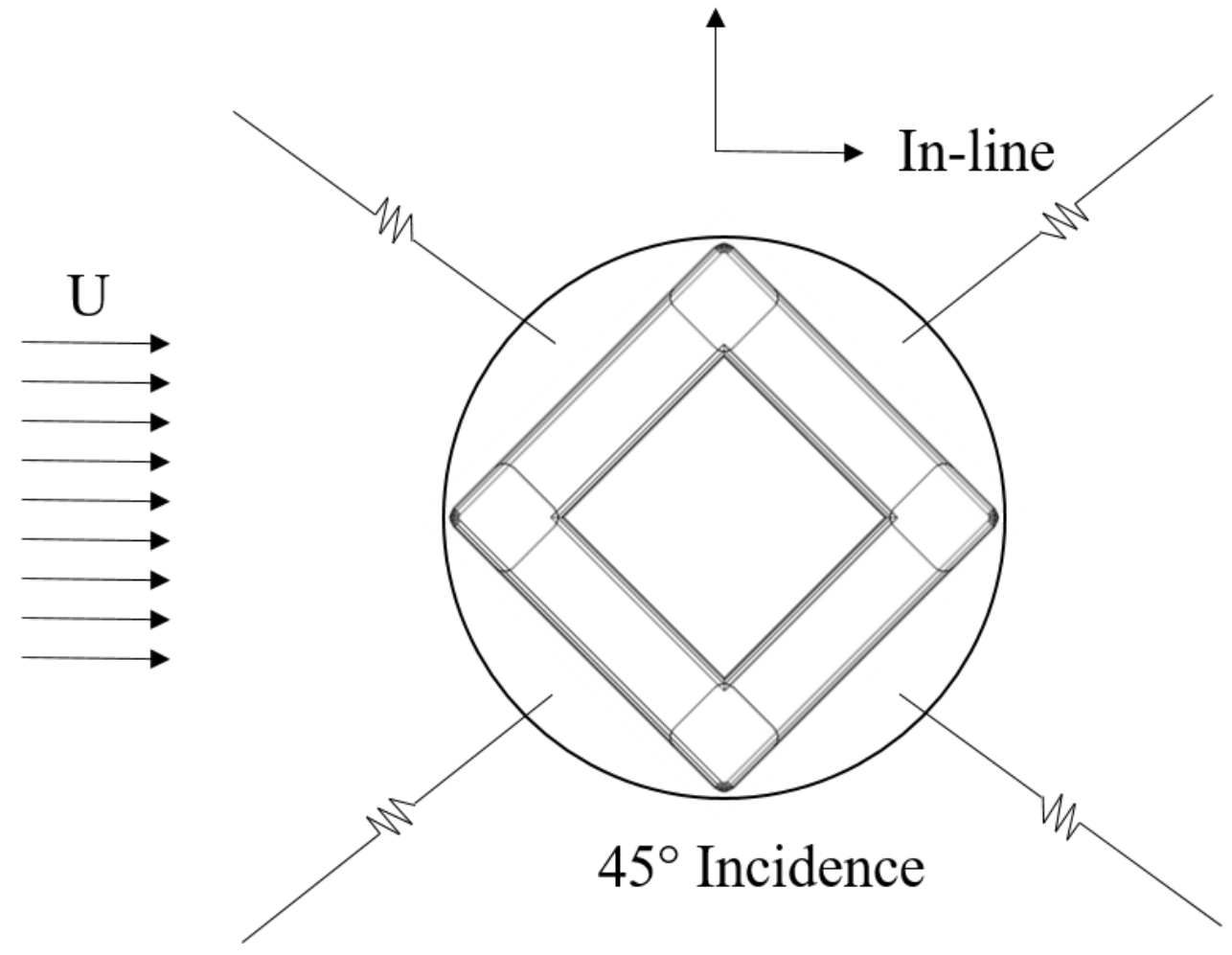

Fig. 2 Schematic of the mooring set-up.

The semi-submersible models with different corner shapes of the column are shown in Fig. 3. The corner ratios for both rounded corner and chamfered corner are $15 \%$ of the column width which is well within a typical range $(10 \% \sim 20 \%)$ for designing the column of offshore platforms. Considering the contribution to buoyancy and the convenience of construction, the design of pontoons, horizontal structural members is kept the same for all three corner shape design. The geometry characteristics of all semi-submersibles are the same except for the corner shape. It is noted that, due to the corners being modified, the projected widths of each column

231 design are slightly different at 45 degree incidence (as shown in Fig. 3). Additionally, 232 the mass ratio (ratio of mass to displacement) is exactly the same for all three models.

233 The Reyonlds number is ranging from $3.6 \times 10^{4}$ to $1.1 \times 10^{5}$ in the present study. 


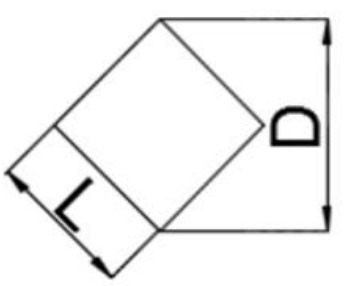

Sharp corners

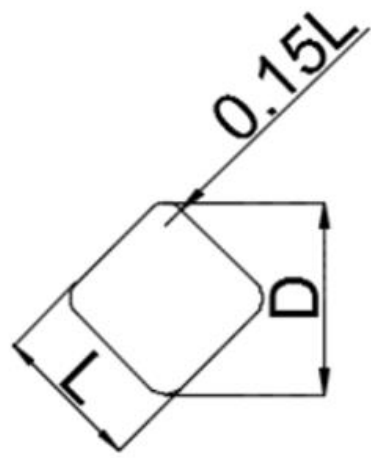

Rounded corners

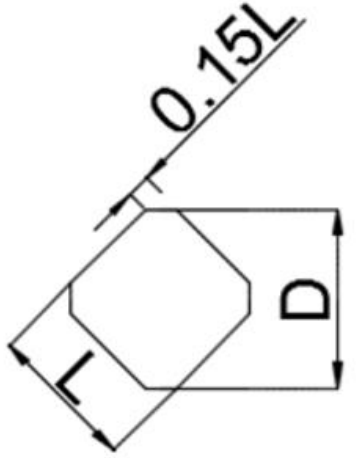

Chamfered corners

Fig. 3 Column sectional configurations.

\subsection{Numerical scheme}

The improved delayed detach eddy simulation (IDDES) model ${ }^{30}$ with the Spalart-Almaras (SA) ${ }^{31}$ was used in this study. IDDES is a model capable of building a single set of formulas both for natural (D)DES applications and for the wall-modelling in large eddy simulation (WMLES) ${ }^{30}$. The delayed detach eddy simulation (DDES) length scale is implemented to eliminate the modelled-stress depletion in the original DES approach, while WMLES is applied to achieve more accurate prediction of the mean velocity in the boundary layer. The boundary layers and irrotational regions are solved using the SA model. However, when the grid is fine enough, it will emulate a basic large eddy simulation (LES) subgrid scale model in the detached flow regions ${ }^{32}$. It is noted that the SA model requires $y^{+}<1$ (where $y^{+}=u * \Delta y_{1} / v$, and where $u *$ denotes

247 the friction velocity at the nearest wall, $\Delta y_{1}$ is the first layer thickness and $v$ is the 248 kinematic viscosity) indicating that the viscous sublayer is properly resolved. All the 249 simulations were carried out using a commercial CFD package, STAR-CCM+9. The 250 finite volume method (FVM) is adopted to discretize the incompressible flow field ${ }^{33}$. 251 The second-order implicit three time levels (ITTL) scheme is applied for the temporal 
252

253

255

discretization. The convective term is evaluated by using a hybrid second-order upwind scheme. The SIMPLE algorithm is employed to treat the pressure and velocity coupling. The governing Navier-Stokes equations solved for the incompressible flow can be written as:

$$
\begin{aligned}
& \nabla \cdot \bar{u}=0 \\
& \frac{\partial \bar{u}}{\partial \bar{t}}+\bar{u} \cdot \nabla \cdot \bar{u}=-\frac{1}{\rho} \nabla p+v \nabla^{2} \bar{u}+\frac{1}{\rho} \nabla \tau
\end{aligned}
$$

where $\nabla$ is the Hamiltonian operator; $u$ is the velocity vector; $t$ is the time; $p$ is the pressure; $\rho$ is the density of water; $v$ is the kinematic viscosity of the water; The last term of Equitation (10) is the Reynolds stress tensor $\tau=-\rho\left(\overline{u^{\prime} u^{\prime}}\right)$, where $u^{\prime}$ denotes the fluctuating velocity. The Reynolds stress tensor is an additional term that represents the effects of turbulence.

\subsection{Computational domain.}

The computational domain size is chosen based on previous experience with modelling vortex-induced motions of the benchmark DDS over a similar parameter space ${ }^{14}$. For all of the simulations, a $9 B_{L} \times 6 B_{L} \times 3 B_{T}$ sized computational domain (see

Fig. 4) was used in the present simulations (where $B_{L}$ is the overall width of the structure and $B_{T}$ is the draft of the structure). More specifically, the domain was considered to be sufficiently large to eliminate both the far field effects from the boundaries and the three-dimensional effects from a spanwise cross flow direction ${ }^{13,14}$. 


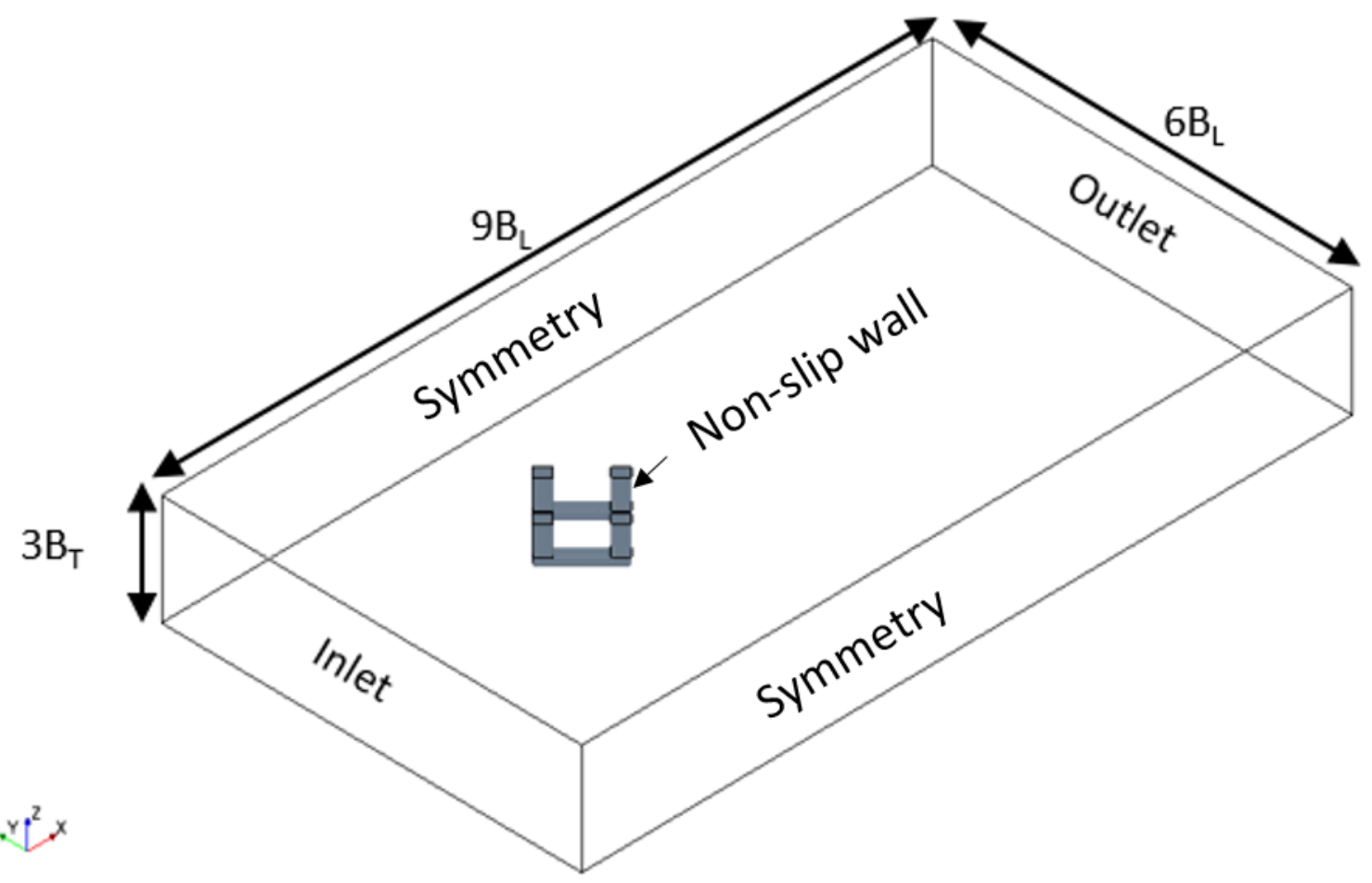

Fig. 4 Computational domain.

The computational domain was modelled with a three-dimensional mesh of

274 elements. A polyhedral mesh ${ }^{32}$ was used in this study. The overall element mesh

275 domain is illustrated in Fig. 5. In the present study, a near wall refinement method

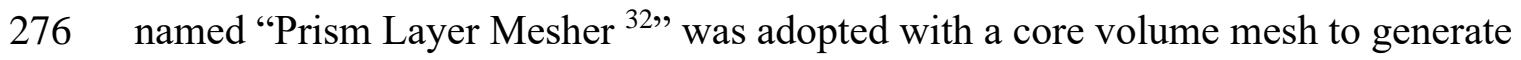

277 orthogonal prismatic cells next to wall surfaces. This layer of cells is necessary to

278 improve the accuracy of the flow solution ${ }^{32}$. The $y^{+}$values were smaller than 1 in all

279 simulations to improve the performance of the boundary layer simulation. Five regional

280 refinements were added in the domain in order to refine both the near wake and the far

281 wake regions. 


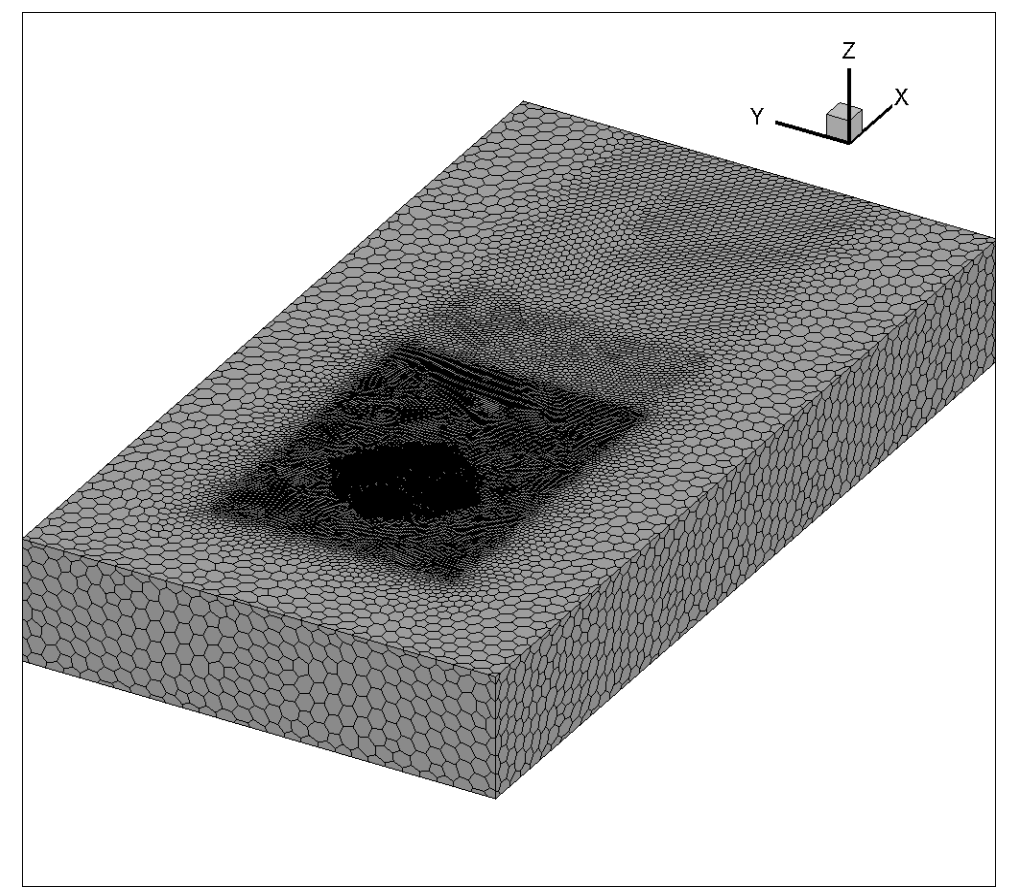

Fig. 5 Visualization of the mesh of the semi-submersible.

The boundary conditions are kept the same in all the simulations. At the inlet, a

285

286

287

288 uniform and constant flow velocity is specified directly for all sensitivity studies. Along the outlet boundary, the pressure is prescribed to be equal to zero. The velocity at the boundary is extrapolated from the interior using reconstruction gradients ${ }^{32}$. For the body surface of the semi-submersible, a no-slip boundary condition is specified ${ }^{32}$. It is noted that the Froude number is quite small $(F r<0.2, F r=U / \sqrt{g D}$, where $U$ is the current velocity, $g$ is the acceleration of gravity and $D$ is the projected width of the column) in all simulations of the present investigation. As observed in the physical model tests ${ }^{13}$, the free surface effects were rather limited and can be ignored. Therefore, only the submerged geometry is considered, and the geometry of the structure above the waterline will not affect the simulation results.

\subsection{Sensitivity study and Validation}


In order to investigate the numerical mesh sensitivity of the calculated results, a

mesh sensitivity study had been carried out with different levels of refinement grids resolution following the guideline proposed by Celik, et al. ${ }^{34}$ at a Reynolds number of $1.1 \times 10^{5}$. Mesh refinement are varied from coarse (with a grid number of $9.4 \times 10^{5}$ ) to fine (with a grid number of $6.9 \times 10^{6}$ ). Additionally, a time step convergence study had been performed with the non-dimensional time step $(\Delta t U / D$, where $\Delta \mathrm{t}$ is the time step,

$U$ is the inlet velocity and $D$ is the projected length of the column) varied from 0.016 to step convergence study can be found in our previous works ${ }^{13,14}$. In the present work, the non-dimensional time step is chosen as 0.008 with a grid number of $3.4 \times 10^{6} 13,14$. Additional convergence test is conducted in the present investigation, namely the numerical model with a rounded corner is further validated with the experimental measurements obtained from the towing tank test ${ }^{14}$. In the present study, the results for all cases were obtained by averaging after more than fifteen FIM oscillation cycles.

Table 2 Validation of the natural periods of the motions in calm water.

\begin{tabular}{lll}
\hline & $\begin{array}{l}\text { Natural period of transverse } \\
\text { motion, } \\
\boldsymbol{T}_{\text {Otransverse }}(\mathbf{s})\end{array}$ & $\begin{array}{l}\text { Natural period of yaw } \\
\text { motion, } \\
\boldsymbol{T}_{\text {Oyaw }}(\mathbf{s})\end{array}$ \\
\hline Numerical & 20.5 & 19.7 \\
Experimental $^{\mathbf{1 4}}$ & 20.1 & 18.3 \\
\hline
\end{tabular}

In Table 2, the natural period obtained from the present numerical model is

312 validated against the experimental data. It is shown that the present numerical model has

313 a good agreement with the experimental results (7.7\% relative variation for yaw motion 314 and $2.0 \%$ relative variation for transverse motion). 
316 agreement with the previous experimental results for both hydrodynamic forces and

317 motion response. Thus, the numerical model can be applied with confidence in future

318 VIM simulations.

(a) transverse motion

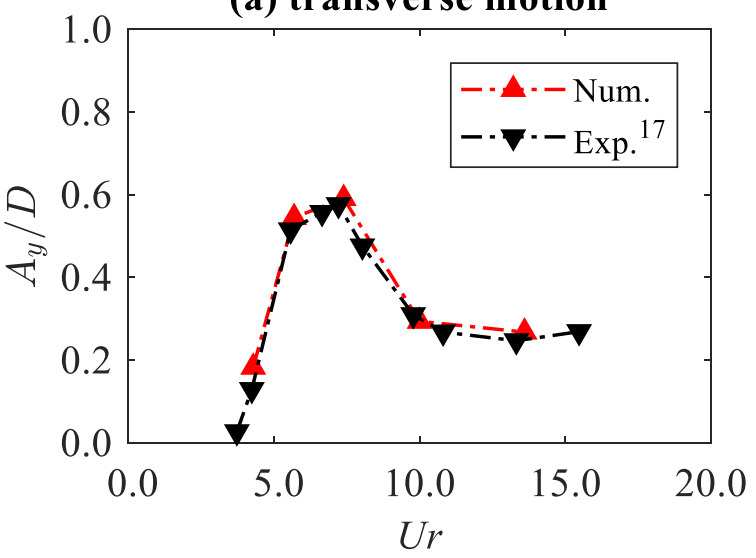

(c) drag force coefficient

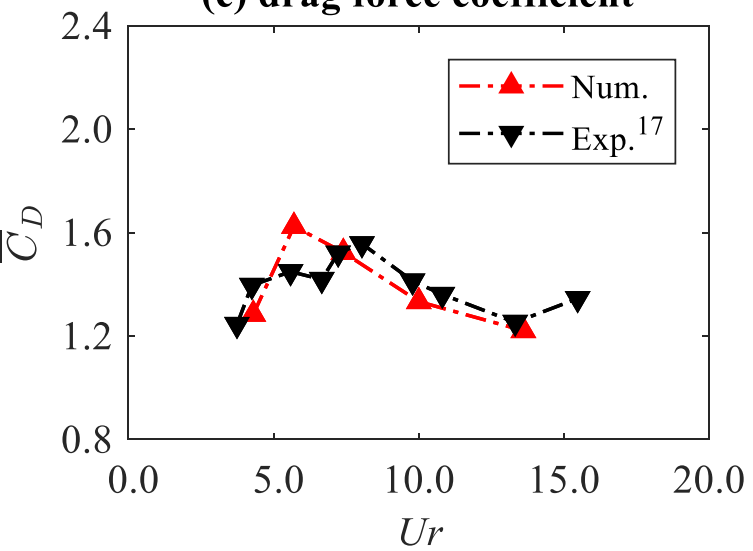

(b) yaw motion

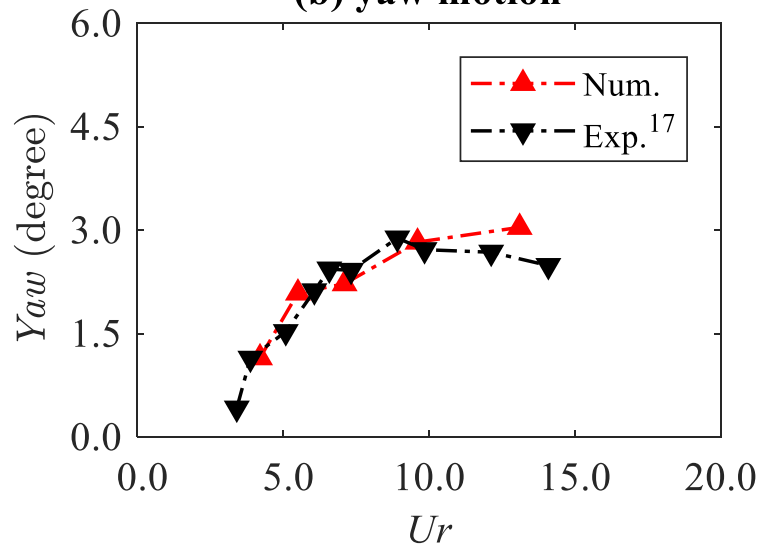

(d) lift force coefficient

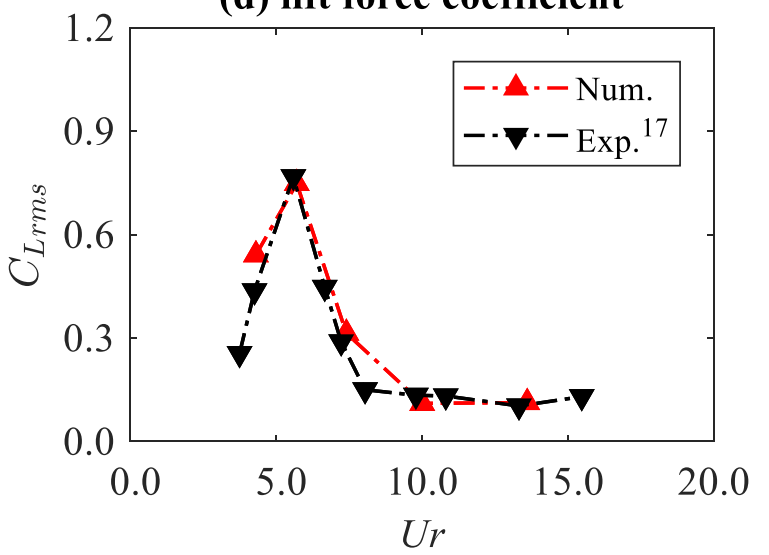

Fig. 6 Validations between the present numerical model (rounded corner) and

321 previous experimental results. (a) non-dimensional transverse amplitude; (b) non-

322 dimensional yaw amplitude; (c) mean drag coefficient; (d) root-mean-square lift

323 coefficient.

\section{Results and discussion}


effects of corner shape. The motion responses, hydrodynamic forces and flow patterns around DDS with columns of three different corner shapes are investigated under five reduced velocities with a current heading of 45 degree. All the results were collected for simulations more than fifteen cycles of the VIM transverse oscillation period in the present study.

Table 3 illustrates the numerical predictions of the natural period of the motions in calm water. It demonstrates that the rounded corner shape structure has the smallest natural period among the three designs while the DDS with sharp corner design has the largest natural period. This is mainly due to the modification of the corner decreasing the hydrodynamic damping of the structure.

Table 3 Natural periods of the motions in calm water.

\begin{tabular}{lll}
\hline Corner shape & $\begin{array}{l}\text { Natural period of transverse } \\
\text { motion, }\end{array}$ & $\begin{array}{l}\text { Natural period of yaw } \\
\text { motion, }\end{array}$ \\
& $\boldsymbol{T}_{\text {otransverse }}(\mathbf{s})$ & $\boldsymbol{T}_{\text {Oyaw }}(\mathbf{s})$ \\
\hline Sharp & 21.3 & 20.3 \\
Rounded & 20.5 & 19.7 \\
Chamfered & 20.6 & 19.7 \\
\hline
\end{tabular}

339 During the FIM process, the energy can be transferred between the fluid flow and the

340 oscillating structure. Motion response and hydrodynamic forces are generated as a result

341 of the energy transformation. In order to gain some deep insights of the transformation

342 process, frequency domain analysis is provided in the present study. The phase angle

343 between the lift coefficient and transverse motion amplitude are further discussed. As a 
344 straightforward way to observe the complex energy transformation, the work done on

345 different structure members are further calculated and presented.

346 4.2.1. Motion characteristics

347 Fig. 7, which compares the numerical results among three different corner shape

348 designs, presents the non-dimensional characteristic amplitudes (transverse, in-line and

349 yaw motions) under 45 degree incidence. As seen in Fig. 7, the largest $A_{y} / D$ for all three

350 design occurs at $U r \approx 7.0$. The "lock-in" region occurs in the range of $6.0 \leq U r \leq 9.0$.

351 The structure with rounded corner shows the most significant motion in the transverse

352 motion. It can be observed that the structure with the sharp corner design has the best

353 transverse motion response among the structures with three different corner shapes.

354 However, as shown in Fig. 7, the non-dimensional transverse amplitudes of the

355 structure with chamfered corner are very close to the rounded corner cases in the "pre

356 lock-in" and "lock-in" regions. Since the project length of the chamfered corner column

357 is $93 \%$ of the rounded corner column's project length, the actual transverse motion

358 response of the structure with chamfered corner is smaller than the rounded corner case

359 in the "pre lock-in" and "lock-in" regions. It is noticed that the chamfered corner case

360 has a rapid increment in the "post lock-in" region for all three horizontal mode motions.

361 In contrast to the sharp and rounded corners, the galloping at $45^{\circ}$ incidence for a square-

362 section shape column was clearly evident when the corner shape modified as chamfered

363 where the motion response increases without self-limiting (see Fig. 7). Regarding the

364 in-line motion, the "lock-in" occurs for a sharp corner structure is found at $U r \approx 9$. By

365 modifying the corner shape, the "lock-in" is shifted to a smaller $U r$. The "lock-in" in the

366 in-line direction occurs for a rounded corner structure is around $U r=7$, while for a

367 chamfered corner structure, the "lock-in" occurs at $U r \approx 6$. It is observed that the 
rounded corner and chamfered corner cases have similar yaw motion responses, and the

369 structure with sharp corner significantly reduced the yaw motion responses.
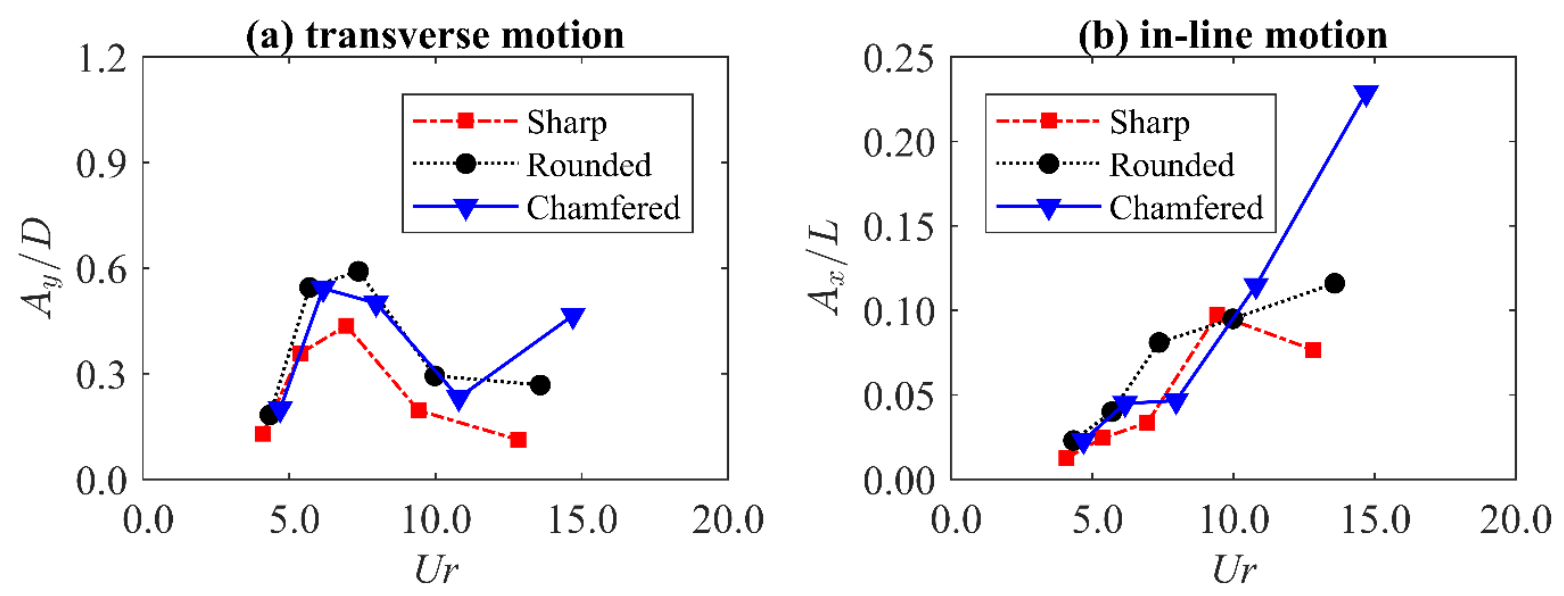

370

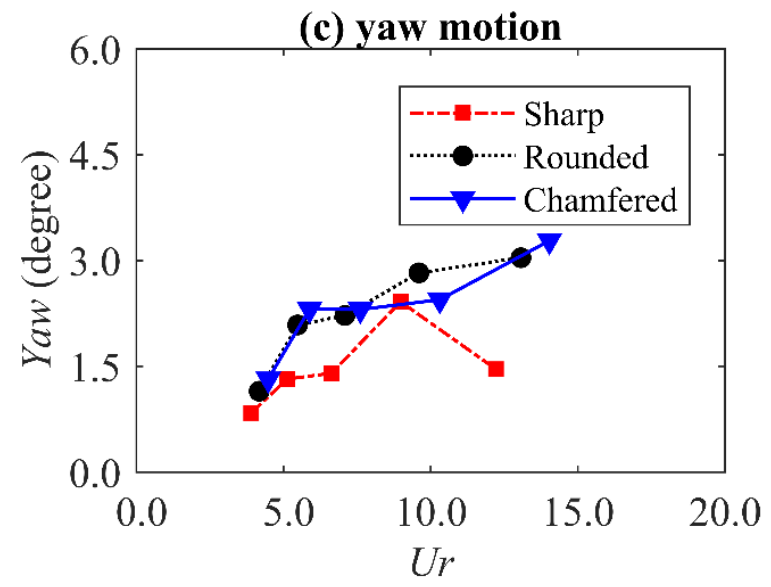

Fig. 7 Non-dimensional transverse, in-line and yaw characteristics amplitudes.

372 (a) Transverse motion; (b) in-line motion; (c) yaw motion.

373 4.2.2. Drag and lift forces on the structure

374

375

376

377

378

379

In addition to the motion responses, the drag and lift coefficient for all three designs are evaluated and shown in Fig. 8. It is clearly observed that, the chamfered corner has the largest $\bar{C}_{D}$ among three corner shape designs. This is due to the chamfered corner has introduced a flat plane into the projected area normal to the current direction. The flat plane at the chamfered corner can increase the drag force on the column. For the lift coefficient, it is shown that the sharp corner case has the minimum $C_{\text {Lrms }}$ which leads 
the structure exhibiting the smallest transverse motion among all three corner shape

381 designs. It is noted that the near-wake flow structure is sensitive to the change of the

382 leading corner design. For a sharp corner column, the flow separation point is fixed on

383 the leading corner edge. However, for a rounded or chamfered corner design, the

384 separation point changes during the motion. As the pressure distribution is altered due

385 to the reattachment on the lateral face strongly influencing the pressure distribution on

386 the column, the fluctuation lift force on the column is changed accordingly. Therefore, it

387 further leads to a structure with a sharp corner showing $C_{L r m s}$ being significantly

388 reduced. As shown in Fig. 8, both $\bar{C}_{D}$ and $C_{L r m s}$ increase when "lock-in" occurs as the

389 consequence of the fluctuations of the force on the structure excited by resonance.

390
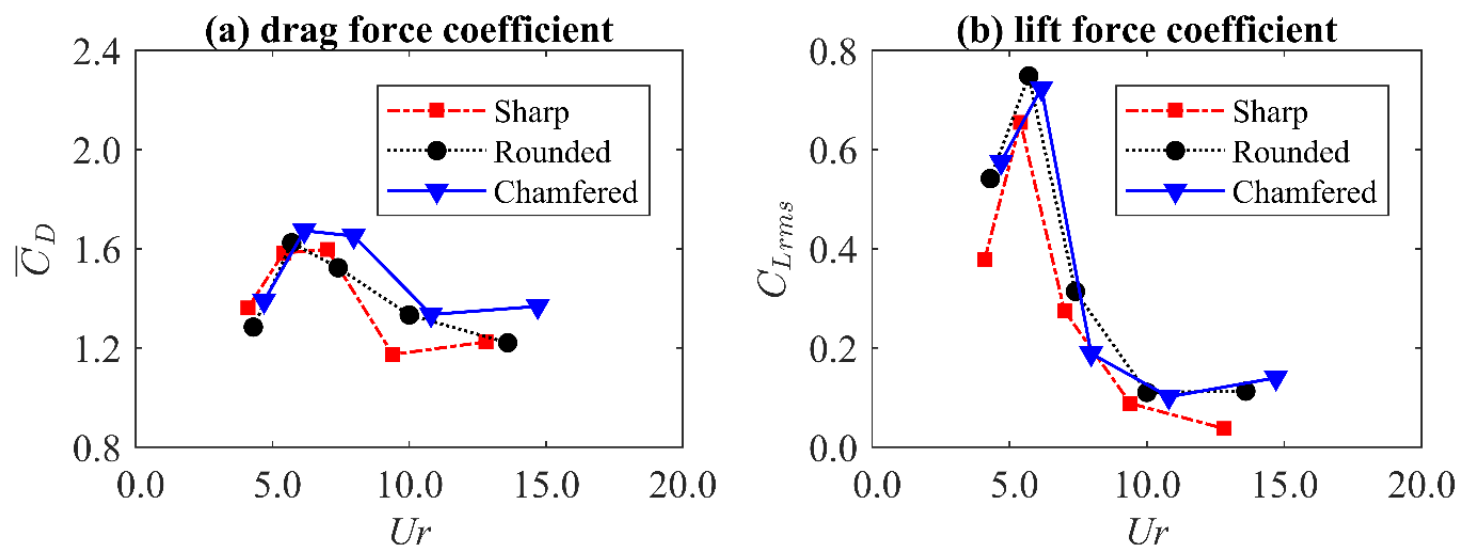

391 Fig. 8 Mean drag coefficient $\left(\bar{C}_{D}\right)$ and root mean square lift coefficient $\left(C_{\text {Lrms }}\right)$. (a) mean drag force coefficient; (b) root mean square lift coefficient.

4.2.3 Motion trajectory and lift coefficient time history.

Fig. 9, Fig. 10 and Fig. 11 present the time history of transverse motion and lift coefficient for three different corner designs respectively. Also shown in the figures are the Power Spectrum Density (PSD) for both transverse motion and lift coefficient 
all three designs are synchronised (have the same phase angle and fluctuation period) with the transverse motions in the "pre lock-in" and "post lock-in". Especially for the

400

401

402

403

404

405

406

407

408

409 sharp corner design, the transverse motion and lift coefficient are fully synchronised when $U r=7$ where the "lock-in" occurs. This indicates that the energy dissipated by the damping is closed to the energy added by the external force. The system therefore reaches its maximum amplitude. However, in the present study, when the motions of the structure shift to the "post lock-in" region, the fluctuation of lift coefficients for all three corner designs are no longer synchronised with the transverse motions. As seen in Fig. 9 (g) (i), Fig. 10 (g) (i) and Fig. 11 (g), a phase delay has been observed in the "post lock-in" region. To further elucidate the response of the structure and the lift force on the structure for various reduced velocities, the power spectrum density of the transverse motions and the lift coefficient are present in Fig. 9, Fig. 10 and Fig. 11. The dominant transverse motion frequency and vortex shedding frequency are both close to the transverse natural frequency in still water at the "pre lock-in" and "lock-in" regions $\left(f_{y} / f_{N}\right.$ and $\left.f_{s} / f_{N} \approx 1\right)$. In addition, a new phenomenon is observed for the chamfered design at $U r=6.2$. Unlike the sharp or rounded corner, in the "lock-in" region, a relatively small peak $(0.1 \%$ amplitude of the dominated peak) is observed in the frequency domain (see Fig. 11 (d)) apart from the dominated peak (especially for the $C_{L}$ ). This indicates that there is a "secondary vortex-shedding" phenomenon existing during the "lock-in". Further discussion based on the flow patterns will be provided in section 4.3 .

In addition to the motion response of and hydrodynamic forces on the structures, the frequency response, as well as the phase angle between the transverse motion and the lift coefficient of the structure, can provide further insight over the energy transfer from 
422 the fluid flow to the structure during VIM. Thus, the non-dimensional response

423 frequency $f_{y} / f_{N}$ and non-dimensional vortex shedding frequency $f_{s} / f_{N}$ are presented in

424 Fig. 12. It is noted that $f_{y} / f_{N}$ and $f_{s} / f_{N}$ are the same at the "pre lock-in" and "lock-in" 425 region for all three different corner designs with a value of approximately 1 . When the

426 VIM shifted to the "post lock-in" region, the non-dimensional vortex shedding

427 frequency is increased and the non-dimensional response frequency is equal to $f_{s} / f_{N}$ at

$428 U r \approx 10$. Beyong $U r \approx 12$, however, $f_{y} / f_{N}$ is evidently bifurcating while $f_{s} / f_{N}$ continues

429 to increase. Two equal weighted peaks (purple circles in the figures) are observed in

430 Fig. 9 (i), Fig. 10(i), and Fig. 11(i). It is noted that the dominant peak $f_{y} / f_{N}$ is decreased,

431 gradually moving away from $f_{S} / f_{N}$ and dropping below the dividing line of $f / f_{N}=1$.

432 However, the significant secondary peak of $f_{y} / f_{N}(*$ marked in Fig. 12) still remains the

433 same as $f_{s} / f_{N}$. 


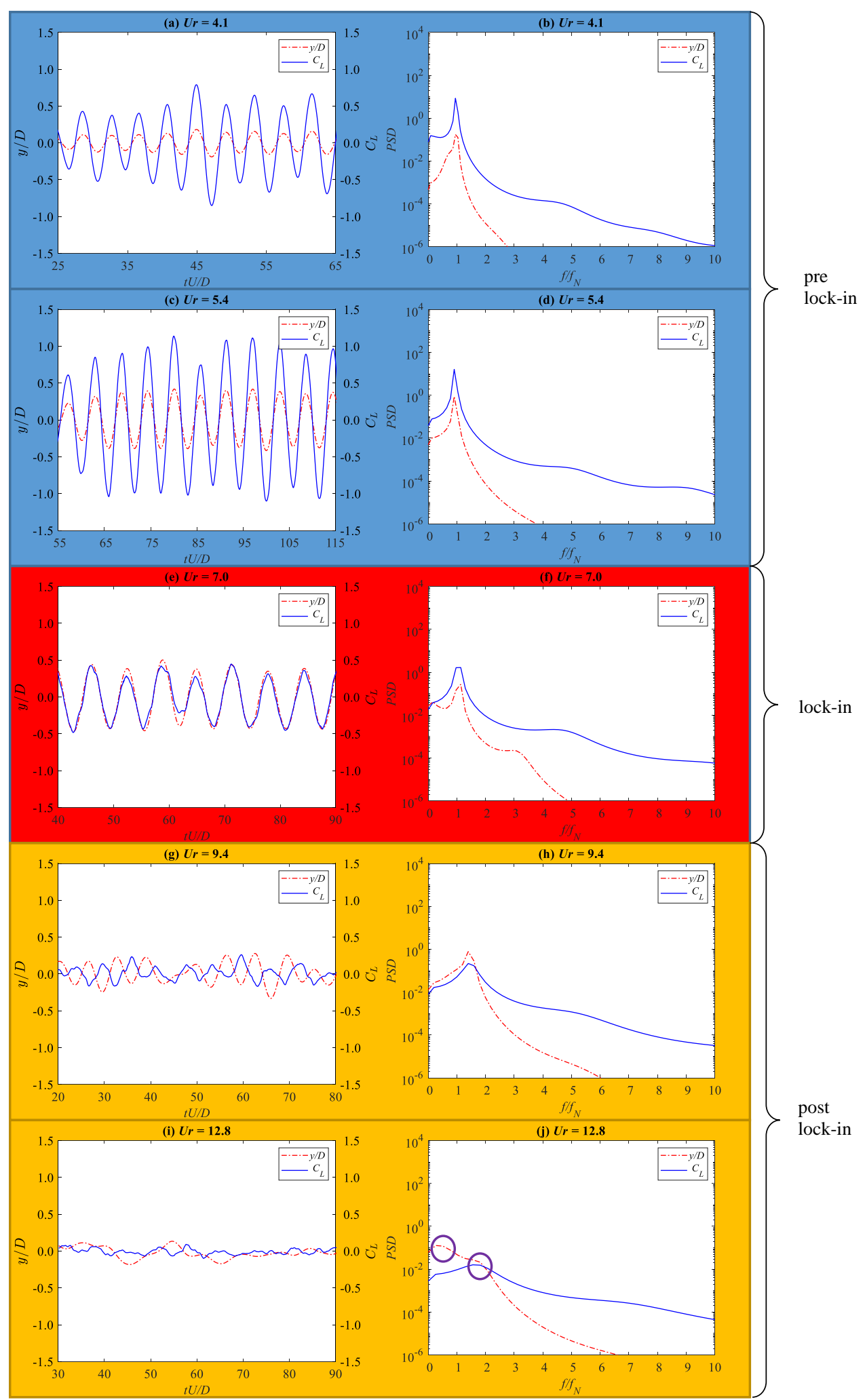

Fig. 9 Time history of lift coefficient and transverse motion for sharp corner

436 design. (a, c, e, g, i are in time domain; b, d, f, h, j are in the frequency domain). (a)

437 Motion trajectory and lift coefficient time history at $U r=4.1$; (b) Motion trajectory and 
438 lift coefficient in frequency domain at $U r=4.1$; (c) Motion trajectory and lift coefficient 439 time history at $U r=5.4$; (d) Motion trajectory and lift coefficient in frequency domain 440 at $U r=5.4$; (e) Motion trajectory and lift coefficient time history at $U r=7.0$; (f) Motion 441 trajectory and lift coefficient in frequency domain at $U r=7.0 ;(\mathrm{g})$ Motion trajectory and 442 lift coefficient time history at $U r=9.4$; (h) Motion trajectory and lift coefficient in 443 frequency domain at $U r=9.4$; (i) Motion trajectory and lift coefficient time history at $444 U r=12.8 ;$ (j) Motion trajectory and lift coefficient in frequency domain at $U r=12.8$. 445 


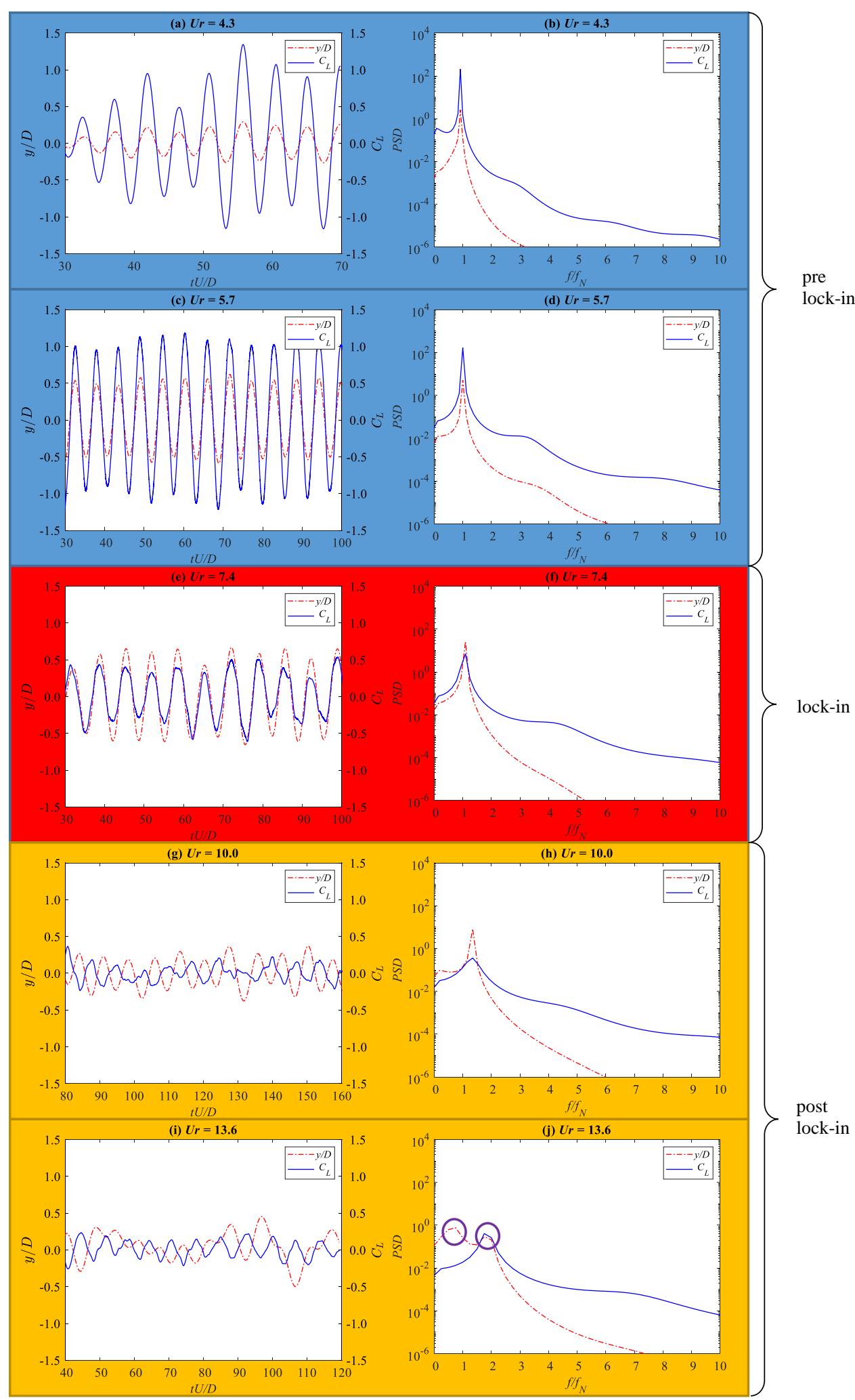

Fig. 10 Time history of lift coefficient and transverse motion for rounded corner 448 design. (a, c, e, g, i are in time domain; b, d, f, h, j are in the frequency domain). (a) 449 Motion trajectory and lift coefficient time history at $U r=4.3$; (b) Motion trajectory and 
450 lift coefficient in frequency domain at $U r=4.3$; (c) Motion trajectory and lift coefficient 451 time history at $U r=5.7$; (d) Motion trajectory and lift coefficient in frequency domain 452 at $U r=5.7$; (e) Motion trajectory and lift coefficient time history at $U r=7.4$; (f) Motion 453 trajectory and lift coefficient in frequency domain at $U r=7.4$; $(\mathrm{g})$ Motion trajectory and 454 lift coefficient time history at $U r=10.0$; (h) Motion trajectory and lift coefficient in 455 frequency domain at $U r=10.0$; (i) Motion trajectory and lift coefficient time history at $456 U r=13.6$; (j) Motion trajectory and lift coefficient in frequency domain at $U r=13.6$. 


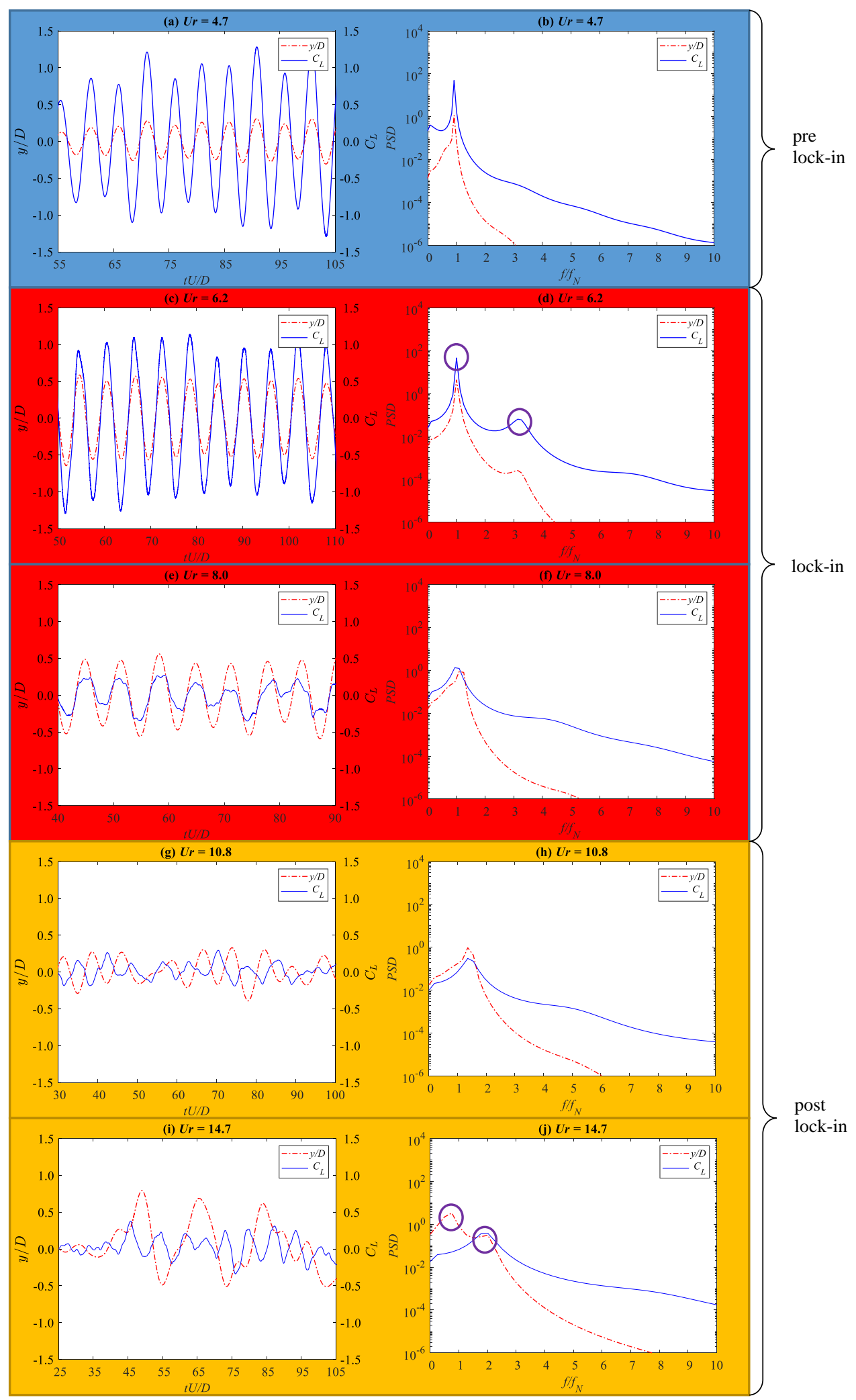

Fig. 11 Time history of lift coefficient and transverse motion for chamfered 459 corner design. (a, c, e, g, i are in time domain; b, d, f, h, j are in the frequency domain). 
460 (a) Motion trajectory and lift coefficient time history at $U r=4.7$; (b) Motion trajectory 461 and lift coefficient in frequency domain at $U r=4.7$; (c) Motion trajectory and lift 462 coefficient time history at $U r=6.2$; (d) Motion trajectory and lift coefficient in 463 frequency domain at $U r=6.2$; (e) Motion trajectory and lift coefficient time history at $464 U r=8.0 ;$ (f) Motion trajectory and lift coefficient in frequency domain at $U r=8.0 ;(\mathrm{g})$ 465 Motion trajectory and lift coefficient time history at $U r=10.8$; (h) Motion trajectory 466 and lift coefficient in frequency domain at $U r=10.8$; (i) Motion trajectory and lift 467 coefficient time history at $U r=14.7$; (j) Motion trajectory and lift coefficient in 468 frequency domain at $U r=14.7$.

469

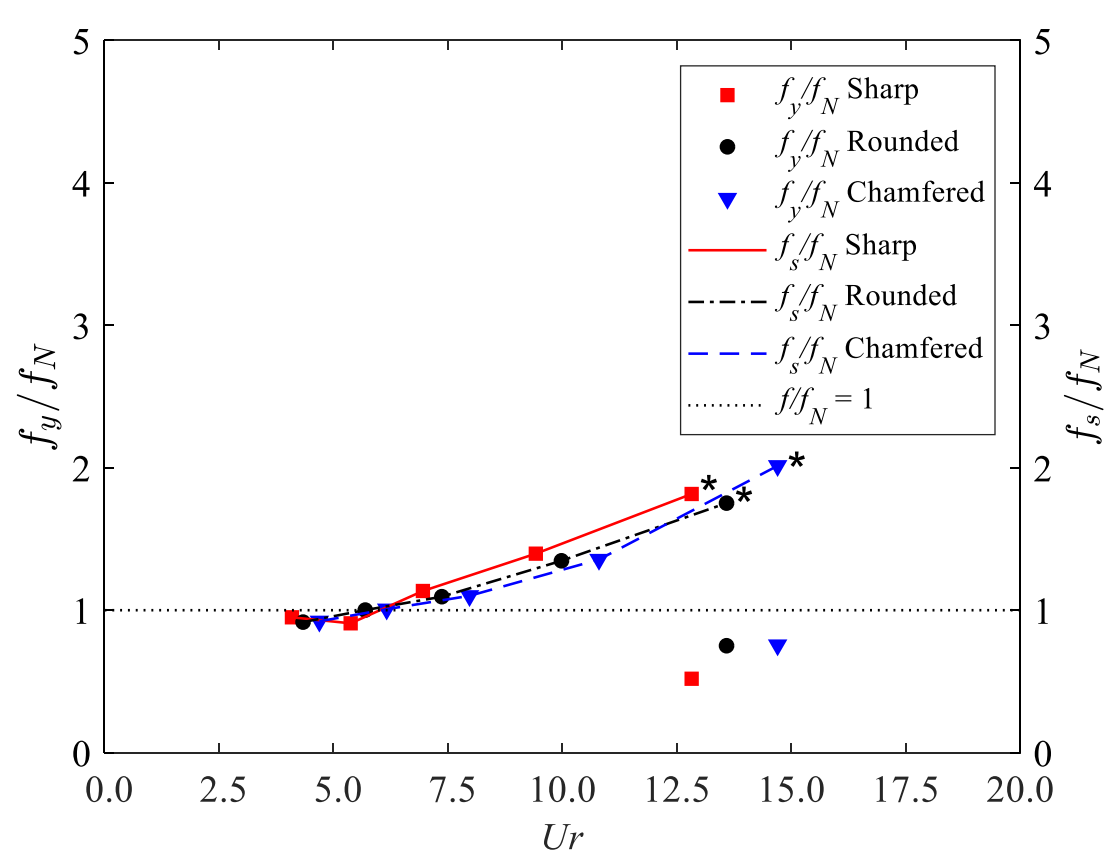

Fig. 12 Non-dimensional transverse response frequency $f_{y} / f_{N}$ and nondimensional vortex shedding frequency $f_{s} / f_{N}(*$ is the secondary peak observed in the frequency domain for $\left.f_{y} / f_{N}\right)$.

Fig. 13, Fig. 14 and Fig. 15 show the motion trajectories in the $X Y$ plane for the structure with different corner designs. Similar to a single cylinder, a typical "8" shaped trajectory is observed in the present study for all corner designs in the "lock-in" region. In the "post lock-in" region, the motion trajectory becomes more chaotic, especially 
477 after $U r=10$. For the chamfered corner design, it is observed that the transverse motion 478 amplitude is approximately $2.4 \%$ higher than the resonance motion amplitude (at $U r=$ 479 6.2) in the "lock-in" region. The galloping at $45^{\circ}$ incidence for a square-section shape 480 column was observed when the corner shape modified as a chamfered corner.

481

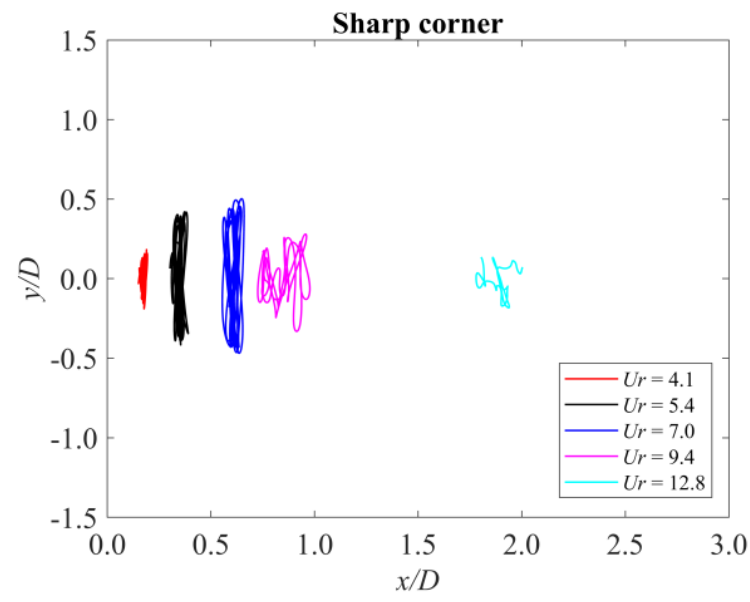

482 Fig. 13 Motion trajectories in the $X Y$ plane for the sharp corner design.

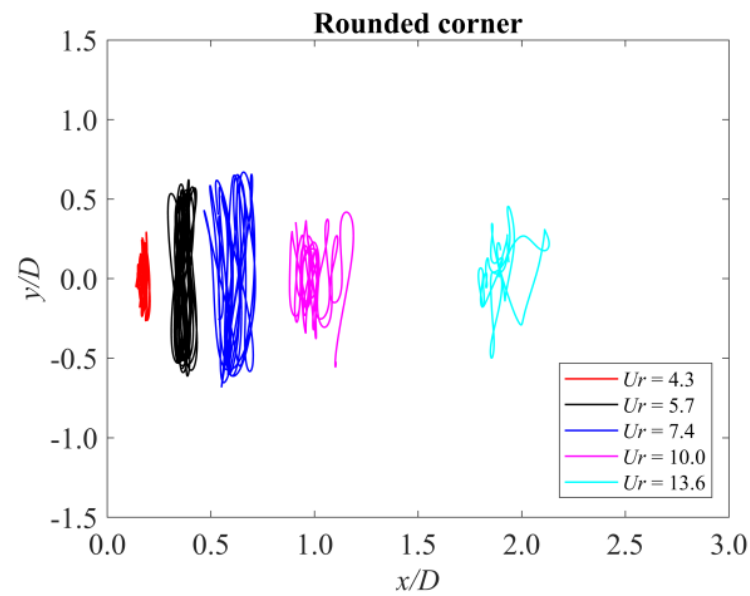

Fig. 14 Motion trajectories in the $X Y$ plane for the rounded corner design. 


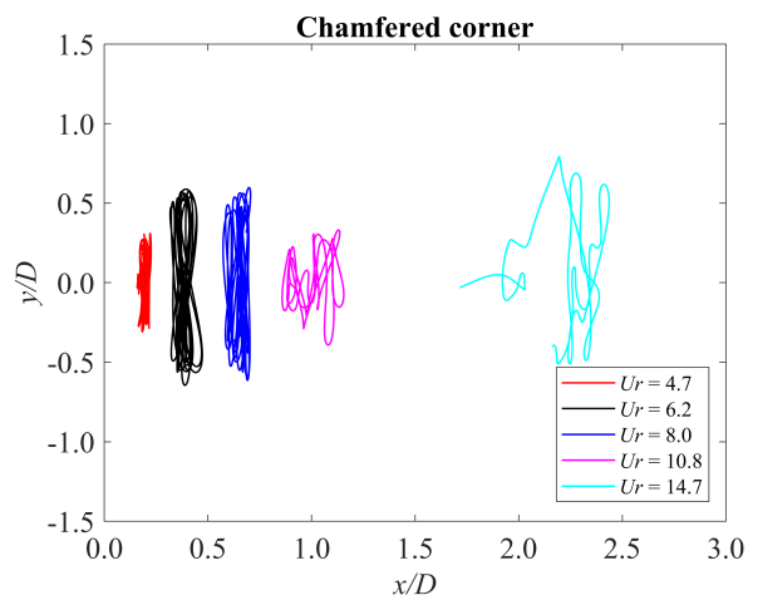

Fig. 15 Motion trajectories in the $X Y$ plane for the chamfered corner design.

487

\subsubsection{Energy transformation during the vortex shedding process}

The phase angle $\left(\emptyset_{C_{L}-A_{y} / L}\right)$ between the lift coefficient and transverse motion amplitude, calculated based on the averaged time lag between the local maximum points of lift coefficient and the transverse motion amplitude, is presented in Fig. 16. In the present study, more than fifteen cycles of the VIM transverse oscillation period are considered for time averaging. The averaged time lag is then multiplied with the frequency of vortex shedding to estimate the phase angle. As seen in Fig. 16, the phase angles in the "pre lock-in" and "lock-in" region are close to zero, and then begin to increase in the "post lock-in" region. After $U r \approx 10$, the phase angle reaches approximately $180^{\circ}$ followed by a rapid decreasing for the sharp and rounded corner designs, indicating a rapid decrease in the transverse motion response. Unlike the sharp and rounded corner designs, a distinct increasing trend of the phase angle is observed along with the reduced velocity for the chamfered corner design, and reaches around $220^{\circ}$ at $U r=14.7$. This increment signifies the large transverse amplitude in the "post lock-in" region for the chamfered corner design. 


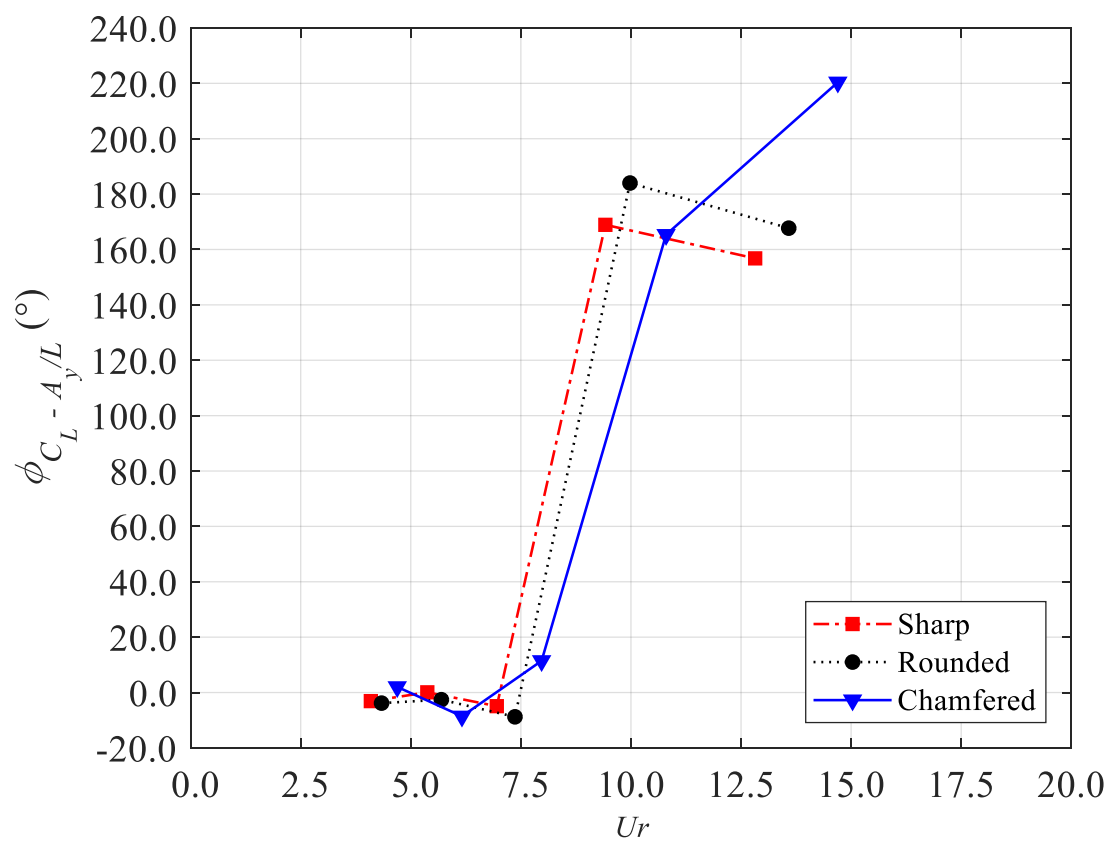

Fig. 16 Phase angle between lift coefficient and transverse amplitude.

504 To examine the complex energy transformation to the structure and the corresponding

505 motion driven parts of the structures, the lift force coefficients and work done on

506 different structure members are further calculated and presented in Fig. 17. As seen in

507 Fig. 17 (a), for a sharp corner design, all the members of the structure are excited due to

508 the "lock-in" phenomenon. However, when the corners are modified as a rounded shape

509 (Fig. 17 (b)), the leading upstream column (Column 1) shows a different trend. The lift

510 coefficient on the upstream column is seen to decrease while an increasing trend is

511 observed for the other members. By changing the corner shape to chamfered, the lift

512 coefficient on the portside column (Column 2) and the starboard side column (Column

513 4) shows a different trend compared with other members of the structure (Fig. 17 (c)).

514 None resonance has been observed on the two side columns. Other members (Column

515 1, Column 3 and pontoons) are excited by the "lock-in" phenomenon. Apart from the

516 force distributions, a straightforward routine to examine the contribution of each 
member to VIM is to determine the work done during the VIM for each member, and

518 the work done by each member of the structure is shown in Fig. 17. The symmetrical

519 characteristics can be clearly identified for all corner design. In addition to the findings

520 made from the previous study ${ }^{13}$, the following new insights can be revealed:

1. Resonance of the work done by the two side columns can be observed for all

522 designs in the present study. However, the resonance is absent to the lift force on the

523 two side columns. Further, for the chamfered corner design, no resonance is observed in

524 both the work done and the lift force in the current study.

2. The work done by the pontoons is highly related to the transverse motion. The pontoon reduces the VIM response throughout the reduced velocity range. In addition, as the transverse motion being more severe, the effect is stronger for the pontoons to restrain the motion in the "lock-in" region.
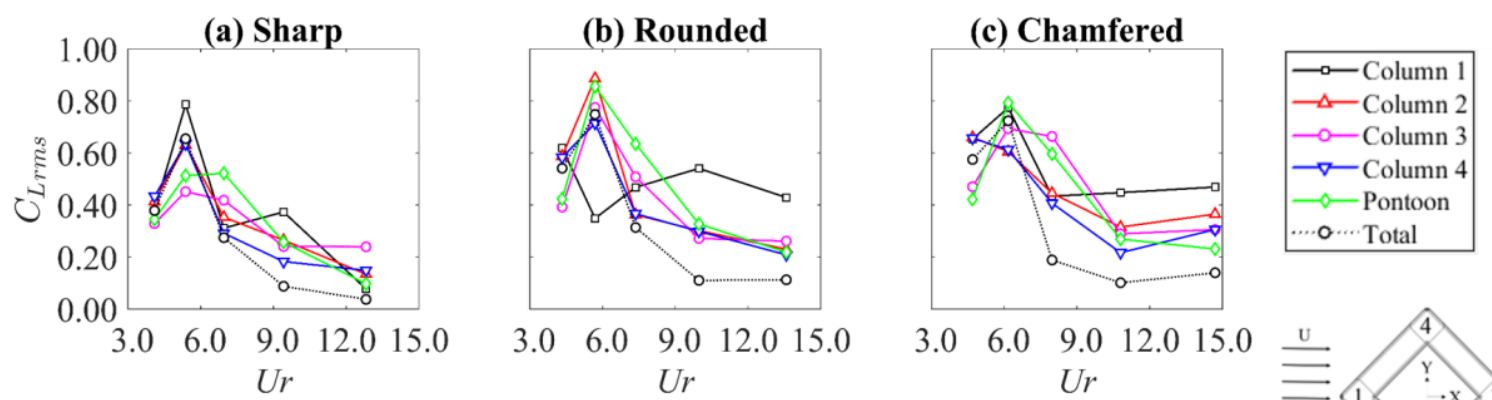

529
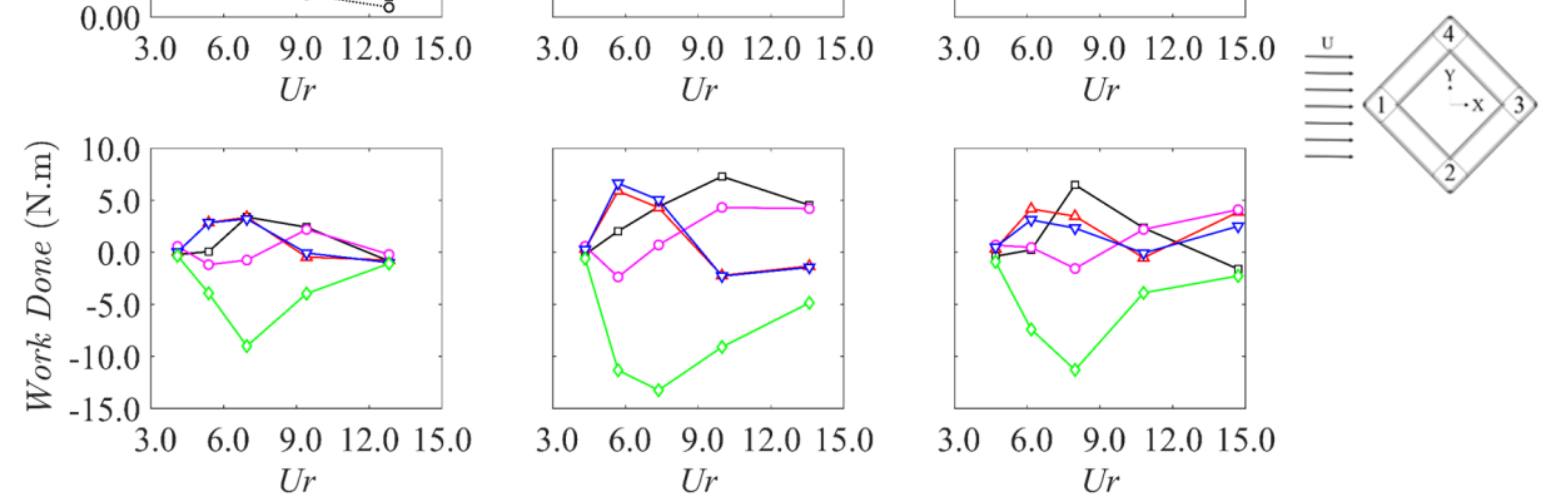

530

Fig. 17 Root mean square lift coefficient $\left(C_{\text {Lrms }}\right)$ and work done on each

531 member of the structure. (a) sharp corner design; (b) rounded corner design; (c)

532 chamfered corner design. 
As seen in Fig. 9, Fig. 10 and Fig. 11, in the "pre lock-in" and "lock-in" region, the motion response frequency $\left(f_{y}\right)$ and vortex shedding frequency $\left(f_{s}\right)$ are both well located around the natural frequency of the structure. This indicates a pure energy transfer from the vortex shedding to the VIM motion of the structure. However, when it shifts to the "post lock-in" region, the motion response frequency $\left(f_{y}\right)$ and vortex shedding frequency $\left(f_{s}\right)$ start to show a different trend with the vortex shedding frequency $\left(f_{s}\right)$ increasing to a higher level while two peaks appearing in the motion responses frequency $\left(f_{y}\right)$ domain.

This indicates a more complex energy transformation between the flow and the structure.

In addition to the phase angle and work done analysis, to provide time-series analysis that can reveal some energy transformation process in the dynamic system, the vortexinduced motions were analysed with continuous wavelet transform (CWT). The CWT provides temporally resolved frequency analysis to give insight into the dynamics of VIM through the time traces in the "post lock-in" region. As seen in Fig. 18, the dominant vortex shedding frequency $\left(f_{s}\right)$ for a sharp corner design is nearly two times of the natural frequency of the structure $\left(f_{N}\right)$. It can be observed that the energy existed during the VIM is relatively low when compared with the other two corner shape designs. This also indicates the extremely small transverse amplitude of the structure. By modifying the corner shape to a rounded corner, the energy contours based on continuous wavelet transform are altered significantly as shown in Fig. 19. It can be seen that, the vortex shedding frequency $\left(f_{s}\right)$ fluctuates around two times of the natural frequency of the structure $\left(f_{N}\right)$. The motion response frequency $\left(f_{y}\right)$ is equally distributed around the natural frequency of the structure $\left(f_{N}\right)$ and at two times of $f_{N}$. In parts of the 
time series, these two equally weighted frequency can be merged together leading to a

557 high energy density as shown in Fig. 19. When the corner shape changed to a

558 chamfered one, further considerable change can be observed for the energy contours

559 shown in Fig. 20. The vortex shedding frequency increases to a higher level where the

560 highest vortex shedding frequency can reach up to more than 3 times of the structural

561 natural frequency $\left(f_{N}\right)$. However, the majority of the energy in the transverse motion

562 response is located at the frequency range lower than the natural frequency $\left(f_{N}\right)$. This

563 indicates the development of the galloping phenomenon. In addition, the energy density

564 of the chamfered corner is much higher than the other two corner shape designs.
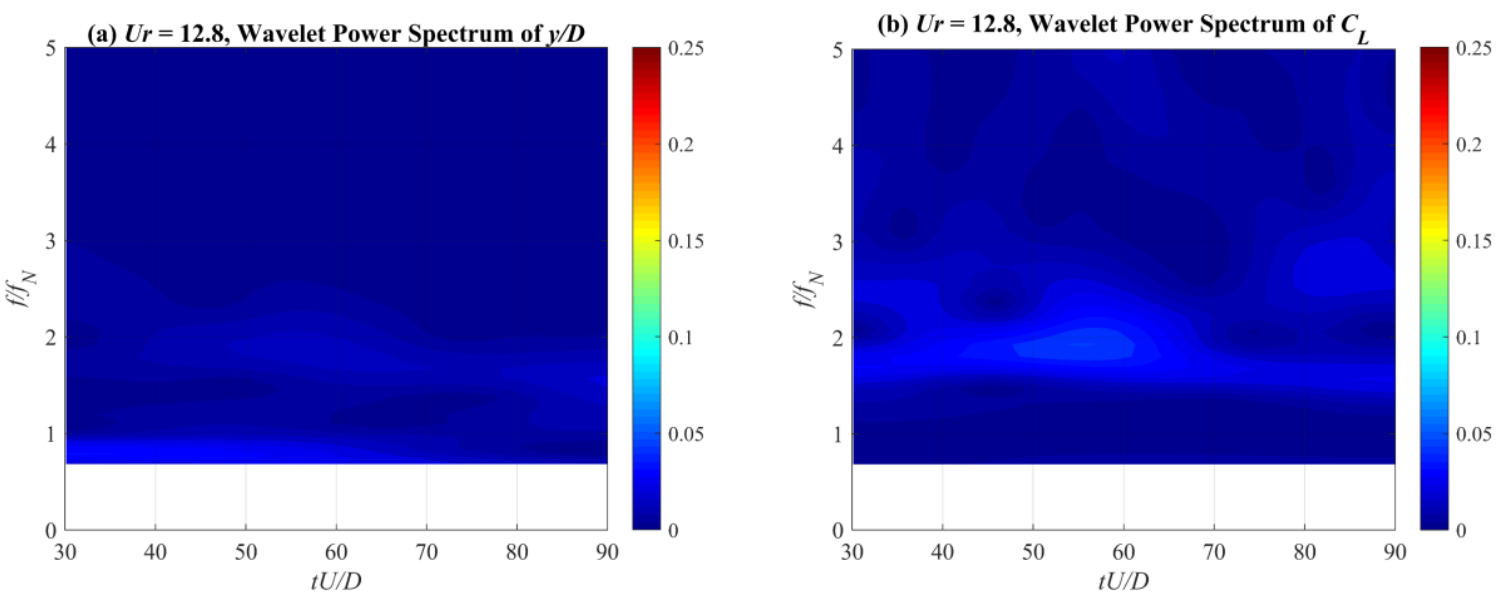

Fig. 18 Time series of the non-dimensional (a) transverse motion response on continuous wavelet transforms in the "post lock-in" region for a sharp corner design. 

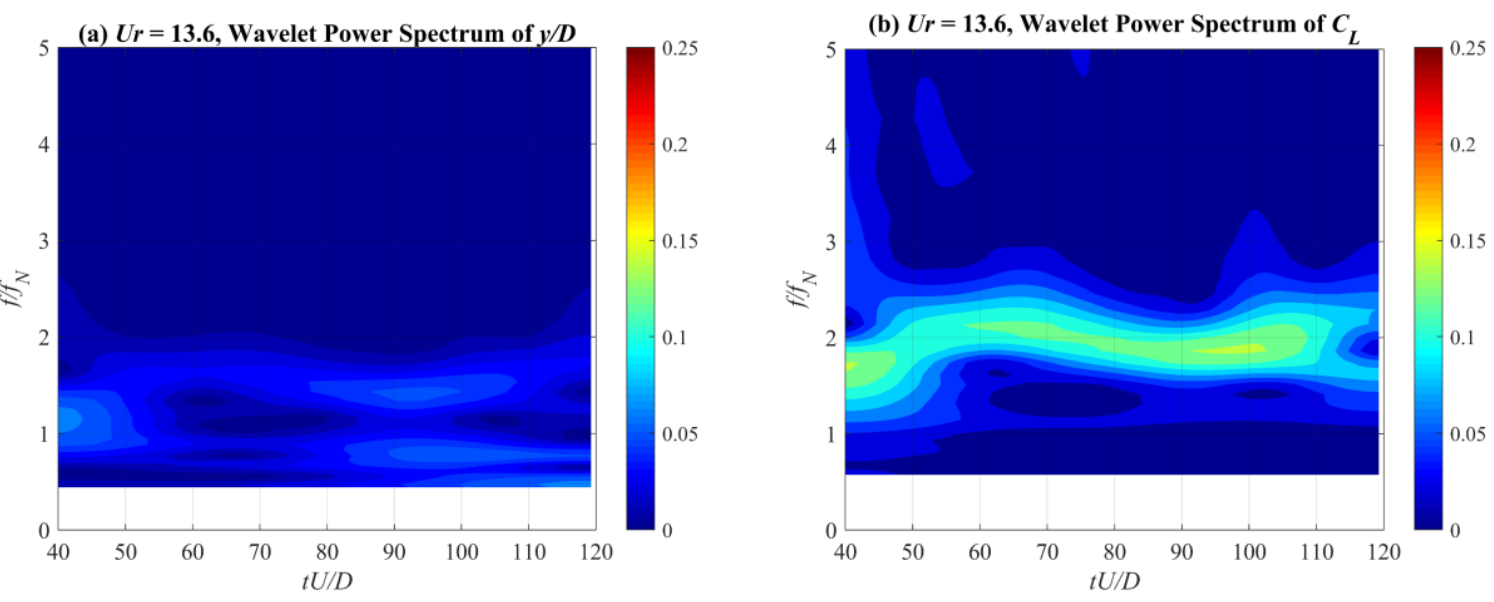

570

Fig. 19 Time series of the non-dimensional (a) transverse motion response

571 frequency and (b) vortex shedding frequency with the frequency energy contours based

572 on continuous wavelet transforms in the "post lock-in" region for a rounded corner 573 design.
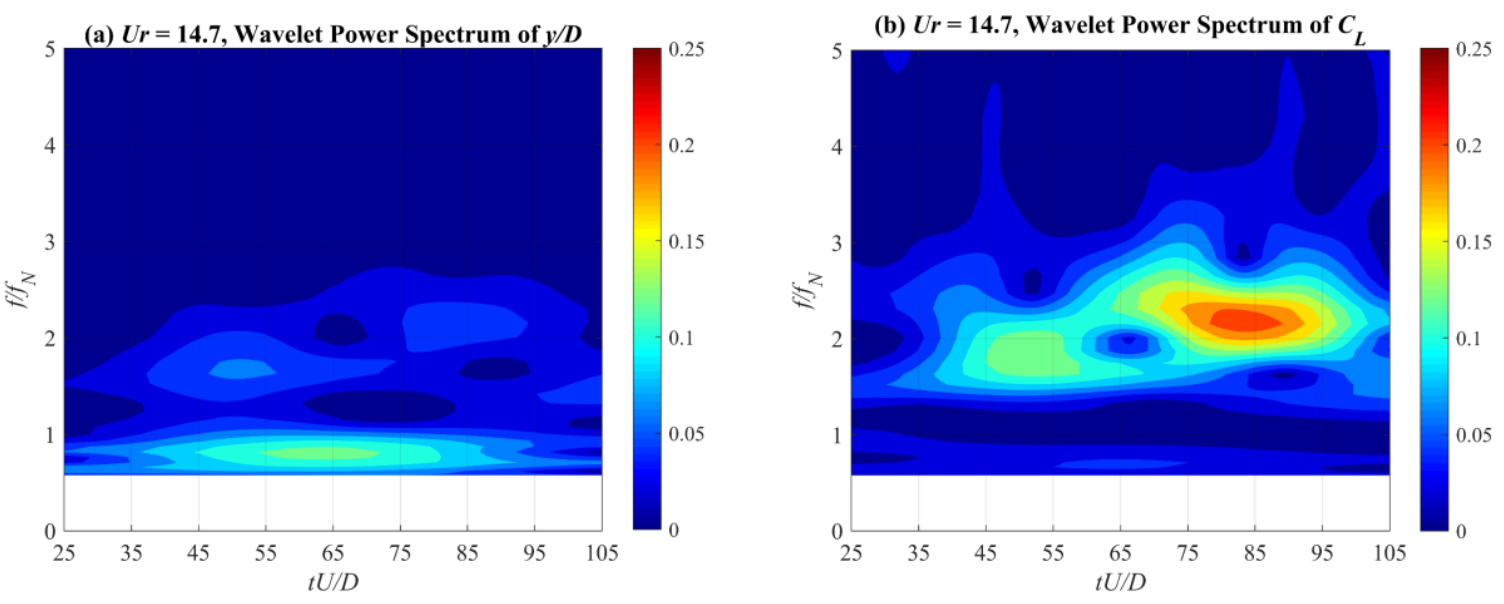

Fig. 20 Time series of the non-dimensional (a) transverse motion response

576 frequency and (b) vortex shedding frequency with the frequency energy contours based

577 on continuous wavelet transforms in the "post lock-in" region for a chamfered corner 578 design.

4.3.1. Instantaneous vorticity and streamline 
582 vorticity contours with instantaneous streamlines in the "lock-in" region are plotted in

583 Fig. 21. The non-dimensional spanwise vorticity is used to describe the vorticity in the

584 present work:

586 where, $\vec{\omega}_{z}$ is the $z$ component of the vorticity, $D$ is the projected length of the column and $U$ is the current speed.

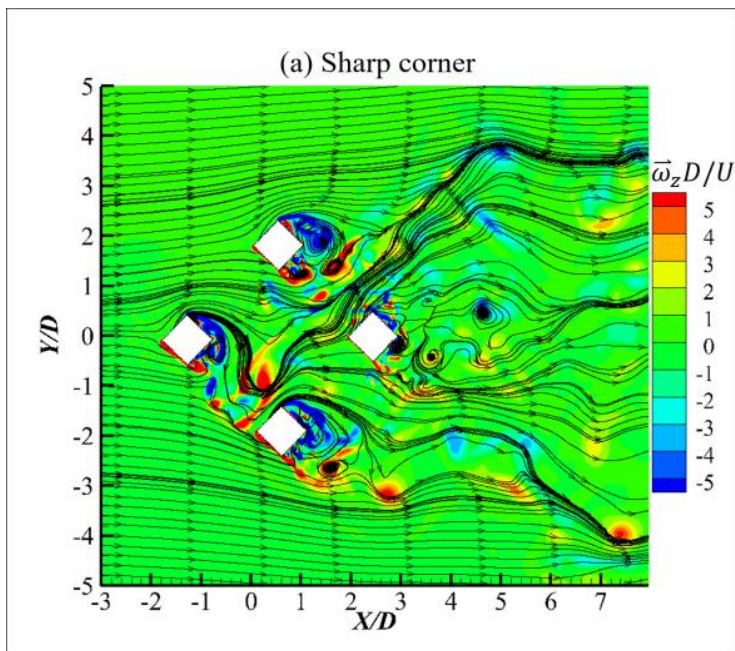

(c) Chamfered corner

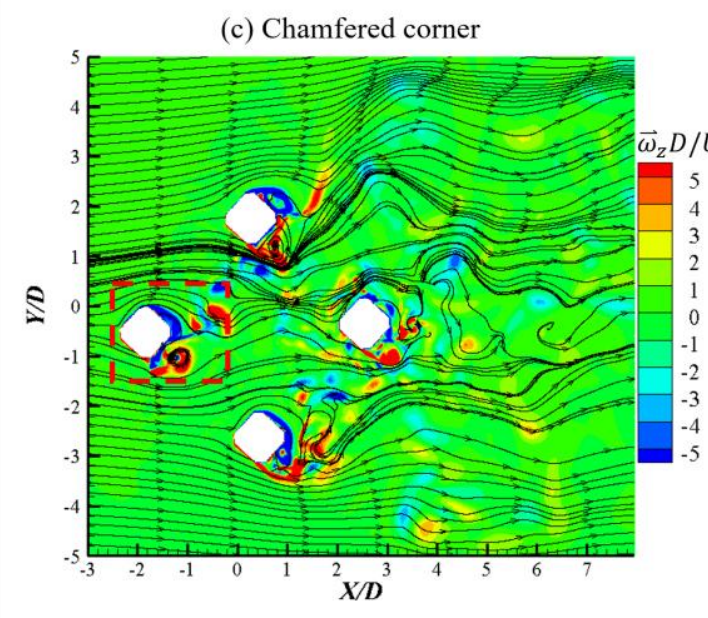

(b) Rounded corner

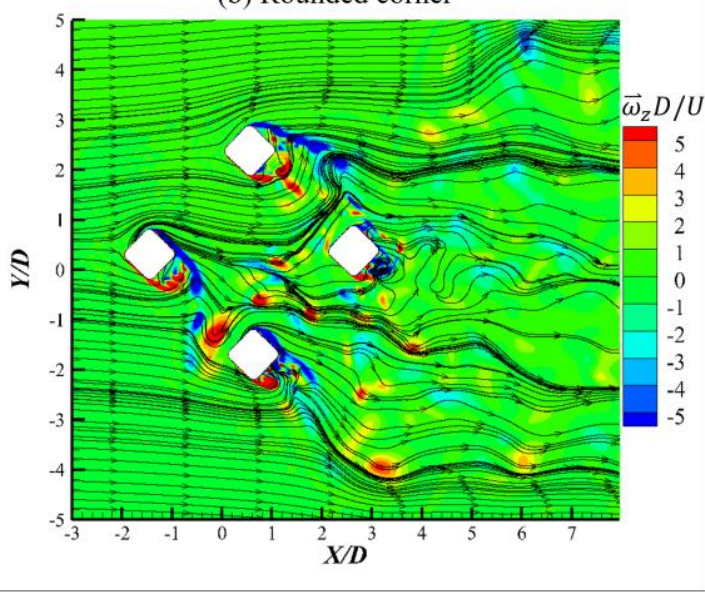

(d) Local zoom in sub figure (c)

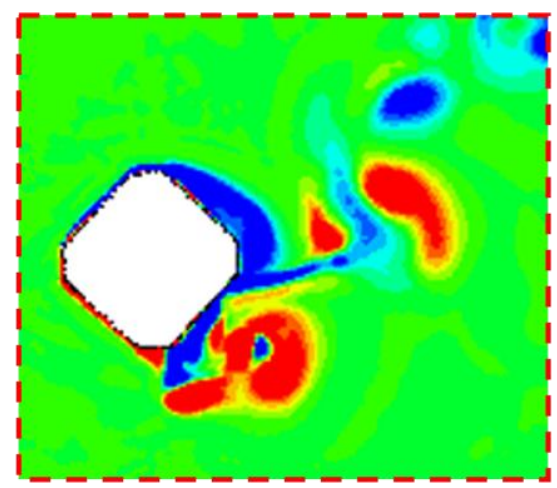

Fig. 21 Instantaneous vorticity contours and streamline for different corner 
592 As seen in Fig. 21, the vortices shed from the upstream corner are finally separated

593 from the side column for both sharp and rounded corner. However, for a chamfered

594 corner shape design case, a unique "re-attached vortex shedding" phenomenon is

595 observed in Fig. 21 (d). The vortices shed from the upstream corner of the column will

596 be separated at one side of the column. However, due to the large amplitude transverse

597 motion and the corner design, it will be "re-attached" to the downstream corner of the

598 column and further reaching to the other side corner of the column. This indicates a

599 higher vortex shedding frequency within one dominated vortex shedding period for the

600 overall structure as shown in Fig. 11(b). This "re-attached" phenomenon has been only

601 observed for the chamfered corner case at the "lock-in" region. It is noted that the

602 energy of this high frequency "re-attach vortex shedding" is extremely small compared

603 with the overall vortex shedding frequency. 


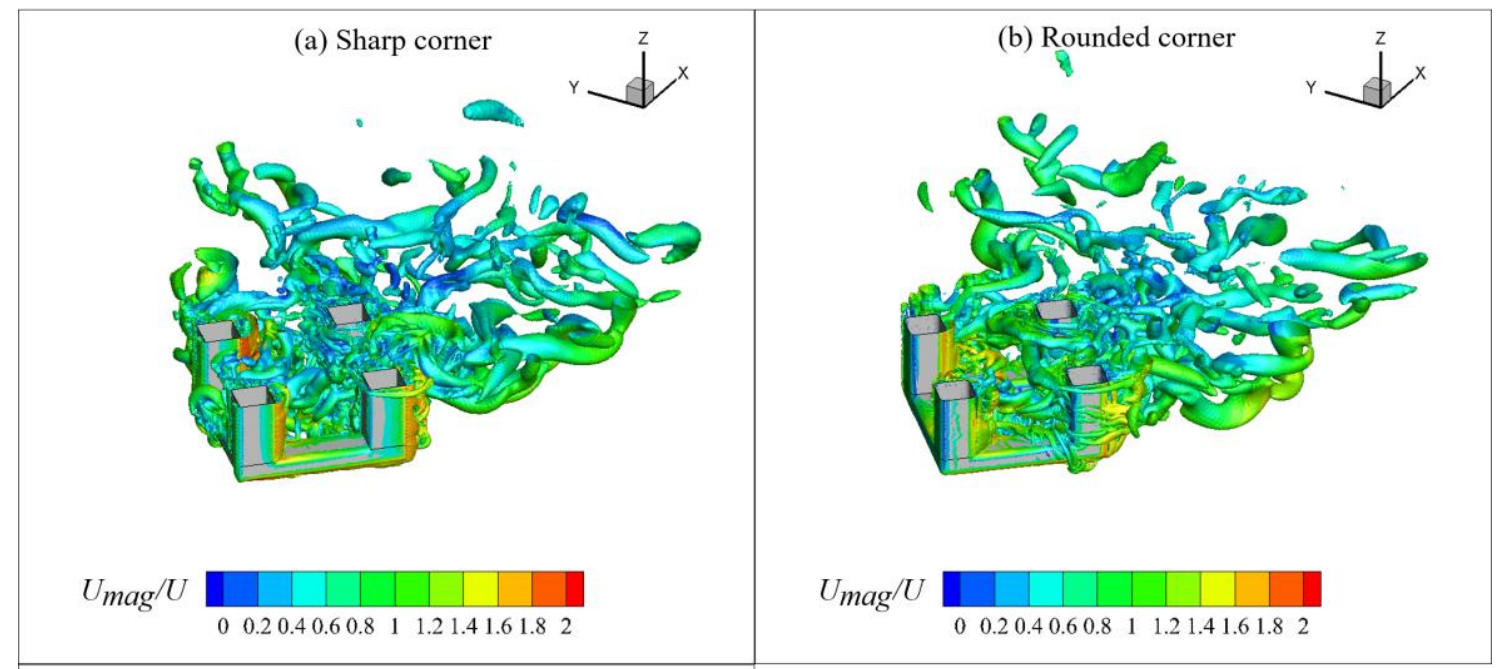

(c) Chamfered corner
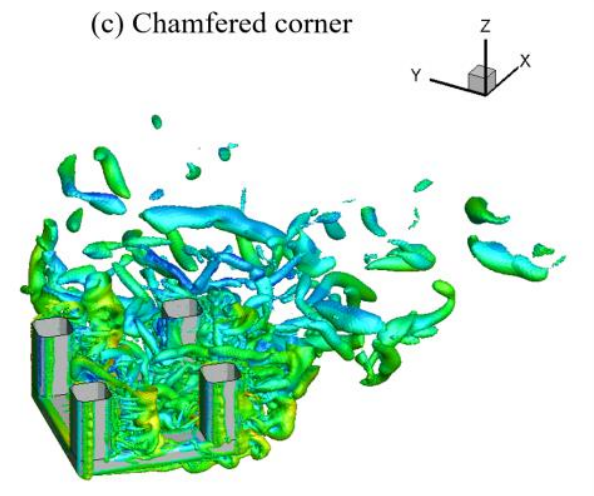

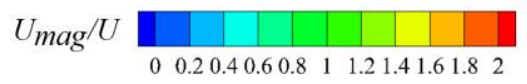

Fig. 22 Isometric view representation of $Q$-criterion of the three different corner 606 design covered by the non-dimensional velocity contours. (a) sharp corner design; (b) 607 rounded corner design; (c) chamfered corner design.

608 To further understand the structures of the wake regions associated with the 609 three different corner shape designs, a vortex identification method based on the $Q$ -

610 criterion has been employed in the present study. Fig. 22 presents the $Q$-criterion based 611 vertical structures for the three corner designs. The isofurfaces are shown at a constant 612 positive value where $Q=0.1$ and covered by the non-dimesnional velocity contours. It 613 can be clearly seen that the sharp corner design only has a single separation point at the 
614 upstream corner while the chamfered corner design has two separation points at the 615 upstream corner.

\section{Conclusions}

617 This paper presents a numerical study focusing on the energy transformation on flow-

618 induced motions of multiple cylindrical structures with various corner shapes. Three

619 different corner shapes were considered, i.e. sharp, rounded and chamfered. The

620 differences of the flow characteristics, the hydrodynamic forces as well as the motion

621 responses are investigated. Based on the relationship between the hydrodynamic forces

622 and the motion responses, the energy transformation between the flow and the structure

623 are further discussed in a perspective of phase angle, work done and frequency energy

624 contours.

625 By examing the characteristics mentioned above, a galloping at $45^{\circ}$ incidence for a

626 square-section shape column is observed when the corner shape modified as a

627 chamfered corner. In addition, a "re-attached vortex shedding" phenomenon is

628 identified when the "lock-in" happened for a chamfered corner design. The cause of this

629 phenomenon is explained by the instantaneous vorticity contours presented in the

630 current study.

631 The analysis of the energy transformation between the flow and the structure revealed

632 that modifying the corner shape had a large effect on the energy transformation leading

633 to a significant change in the hydrodynamic forces and the FIM motion responses.

634 This study focuses on the 45 degree flow incidence, hence more incidences should be

635 considered and examined in order to obtain a more generalized understanding of the

636 energy transformation process during FIM of a multi-column floating structure. 
Acknowledgement

The authors would like to acknowledge the support of Newton Fund of Royal Academy of Engineering UK (NRCP/1415/211). This work made use of the facilities of N8 HPC Centre of Excellence, provided and funded by the N8 consortium and EPSRC (Grant No.EP/K000225/1).

\section{References}

1. T. Kokkinis, R. E. Sandström, H. T. Jones, H. M. Thompson, and W. L. Greiner, Development of a Stepped Line Tensioning Solution for Mitigating VIM Effects in Loop Eddy Currents for the Genesis Spar (American Society of Mechanical Engineers, 2004).

2. A. L. C. Fujarra, G. F. Rosetti, J. de Wilde, and R. T. Gonçalves, State-of-art on vortex-induced motion: a comprehensive survey after more than one decade of experimental investigation (American Society of Mechanical Engineers, 2012). 3. O. J. Waals, A. C. Phadke, and S. Bultema, Flow Induced Motions on Multi Column Floaters (American Society of Mechanical Engineers, 2007).

4. O. Rijken, and S. Leverette, Field measurements of vortex induced motions of a deep draft semisubmersible (American Society of Mechanical Engineers, 2009).

5. J. Den Hartog, "Transmission line vibration due to sleet," Transactions of the American Institute of Electrical Engineers 51, 1074 (1932).

6. B. Liu, and R. K. Jaiman, "Interaction dynamics of gap flow with vortex-induced vibration in side-by-side cylinder arrangement," Physics of Fluids 28, 127103 (2016). 7. Z. Li, R. K. Jaiman, and B. C. Khoo, "Coupled dynamics of vortexinduced vibration and stationary wall at low Reynolds number," Physics of Fluids 29, 093601 (2017).

8. W. Yao, and R. Jaiman, "Stability analysis of the wake-induced vibration of tandem circular and square cylinders," Nonlinear Dynamics 95, 13 (2019). 
669

670

671

672

673

674

675

676

677

678

679

680

681

682

683

684

685

686

687

688

689

690

691

692

693

694

695

696

697

698

699

700

701

702

703

704

705

706

9. L. Shen, and Z. Sun, "Jump phenomena in vortex-induced vibrations of a circular cylinder at a low Reynolds number," Physics of Fluids 31, 123605 (2019).

10. H. Zhu, C. Zhang, and W. Liu, "Wake-induced vibration of a circular cylinder at a low Reynolds number of 100," Physics of Fluids 31, 073606 (2019).

11. H. Jiao, and G. Wu, "Free vibration predicted using forced oscillation in the lock-in region," Physics of Fluids 30, 113601 (2018). 12. M. Rastan, A. Sohankar, and M. M. Alam, "Low-Reynoldsnumber flow around a wall-mounted square cylinder: Flow structures and onset of vortex shedding," Physics of Fluids 29, 103601 (2017). 13. Y. Liang, and L. Tao, "Interaction of vortex shedding processes on flow over a deep-draft semi-submersible," Ocean Engineering 141, 427 (2017).

14. Y. Liang, L. Tao, L. Xiao, and M. Liu, "Experimental and numerical study on vortex-induced motions of a deep-draft semisubmersible," Applied Ocean Research 67, 169 (2017).

15. P. W. Bearman, J. M. R. Graham, E. D. Obasaju, and G. M. Drossopoulos, "The influence of corner radius on the forces experienced by cylindrical bluff bodies in oscillatory flow," Applied Ocean Research 6, 83 (1984).

16. J. C. Hu, Y. Zhou, and C. Dalton, "Effects of the corner radius on the near wake of a square prism," Experiments in Fluids 40, 106 (2005).

17. M. Liu, L. Xiao, J. Yang, and X. Tian, "Parametric study on the vortex-induced motions of semi-submersibles: Effect of rounded ratios of the column and pontoon," Physics of Fluids 29, 055101 (2017).

18. T. Tamura, T. Miyagi, and T. Kitagishi, "Numerical prediction of unsteady pressures on a square cylinder with various corner shapes," Journal of Wind Engineering and Industrial Aerodynamics 74, 531 (1998).

19. T. Tamura, and T. Miyagi, "The effect of turbulence on aerodynamic forces on a square cylinder with various corner shapes," Journal of Wind Engineering and Industrial Aerodynamics 83, 135 (1999).

20. Y. Cao, and T. Tamura, "Supercritical flows past a square cylinder with rounded corners," Physics of Fluids 29, 085110 (2017). 
21. J. Zhao, A. Nemes, D. Lo Jacono, and J. Sheridan, "Branch/mode competition in the flow-induced vibration of a square cylinder," Philosophical Transactions of the Royal Society A: Mathematical, Physical and Engineering Sciences 376, 20170243 (2018).

22. J. Zhao, J. S. Leontini, D. L. Jacono, and J. Sheridan, "Fluidstructure interaction of a square cylinder at different angles of attack," Journal of Fluid Mechanics 747, 688 (2014).

23. R. T. Gonçalves, G. F. Rosetti, A. L. C. Fujarra, and A. C. Oliveira, "Experimental study on vortex-induced motions of a semisubmersible platform with four square columns, Part I: Effects of current incidence angle and hull appendages," Ocean Engineering 54, 150 (2012).

24. A. Antony, V. Vinayan, S. Madhavan, A. Parambath, J. Halkyard, J. Sterenborg, S. Holmes, D. Spernjak, S. J. Kim, and W. Head, VIM Model Test of Deep Draft Semisubmersibles Including Effects of Damping (Offshore Technology Conference, 2016). 25. Y. Liang, and L. Tao, "Interaction of vortex shedding processes on flow over a deep-draft semi-submersible," Ocean Engineering (2017). 26. G. Schewe, "On the force fluctuations acting on a circular cylinder in crossflow from subcritical up to transcritical Reynolds numbers," Journal of Fluid Mechanics 133, 265 (1983).

27. C. Norberg, "Flow around rectangular cylinders: pressure forces and wake frequencies," Journal of wind engineering and industrial aerodynamics 49, 187 (1993).

28. T. Sarpkaya, "A critical review of the intrinsic nature of vortexinduced vibrations," Journal of Fluids and Structures 19, 389 (2004). 29. R. T. Gonçalves, G. F. Rosetti, A. L. C. Fujarra, and A. C. Oliveira, "Experimental study on vortex-induced motions of a semisubmersible platform with four square columns, Part II: Effects of surface waves, external damping and draft condition," Ocean Engineering 62, 10 (2013).

30. M. L. Shur, P. R. Spalart, M. K. Strelets, and A. K. Travin, "A hybrid RANS-LES approach with delayed-DES and wall-modelled LES capabilities," International Journal of Heat and Fluid Flow 29, 1638 (2008).

31. P. R. Spalart, W. H. Jou, M. Strelets, and S. R. Allmaras, "Comments on the feasibility of LES for wings, and on a hybrid RANS/LES approach," Advances in DNS/LES 1, 4 (1997). 
745 32. CD-adapco, User Guide (Star-CCM+ Version 9.04, 2014).

746 33. J. Blazek, Computational fluid dynamics: principles and

747 applications (Butterworth-Heinemann, 2015).

748 34. I. B. Celik, U. Ghia, and P. J. Roache, "Procedure for estimation

749 and reporting of uncertainty due to discretization in CFD

750 applications," Journal of Fluids Engineering-Transactions of the

751 ASME 130, (2008).

752 


\section{List of Figures}

Fig. 1 Numerical model (rounded corner as an example) simulated in the present study ( $\mathrm{A}$ is the entire model; $\mathrm{B}$ is the decomposed model which shows the definition of the individual members; $\mathrm{C}$ is the sketch of the semi-submersible).

Fig. 2 Schematic of the mooring set-up.

Fig. 3 Column sectional configurations.

Fig. 4 Computational domain.

Fig. 5 Visualization of the mesh of the semi-submersible. 16

Fig. 6 Validations between the present numerical model (rounded corner) and previous experimental results. (a) non-dimensional transverse amplitude; (b) nondimensional yaw amplitude; (c) mean drag coefficient; (d) root-mean-square lift coefficient.

Fig. 7 Non-dimensional transverse, in-line and yaw characteristics amplitudes.

(a) Transverse motion; (b) in-line motion; (c) yaw motion.

Fig. 8 Mean drag coefficient $(C D)$ and root mean square lift coefficient (CLrms). (a) mean drag force coefficient; (b) root mean square lift coefficient.

Fig. 9 Time history of lift coefficient and transverse motion for sharp corner design. (Both in time and frequency domain). (a) Motion trajectory and lift coefficient time history at $U r=4.1$; (b) Motion trajectory and lift coefficient in frequency domain at $U r=4.1$; (c) Motion trajectory and lift coefficient time history at $U r=5.4$; (d)

Motion trajectory and lift coefficient in frequency domain at $U r=5.4$; (e) Motion trajectory and lift coefficient time history at $U r=7.0$; (f) Motion trajectory and lift coefficient in frequency domain at $U r=7.0 ;(\mathrm{g})$ Motion trajectory and lift coefficient time history at $U r=9.4$; (h) Motion trajectory and lift coefficient in frequency domain 
at $U r=9.4$; (i) Motion trajectory and lift coefficient time history at $U r=12.8$; (j)

Motion trajectory and lift coefficient in frequency domain at $U r=12.8$.

Fig. 10 Time history of lift coefficient and transverse motion for rounded corner design. (Both in time and frequency domain). (a) Motion trajectory and lift coefficient time history at $U r=4.3$; (b) Motion trajectory and lift coefficient in frequency domain at $U r=4.3$; (c) Motion trajectory and lift coefficient time history at $U r=5.7$; (d) Motion trajectory and lift coefficient in frequency domain at $U r=5.7$; (e) Motion trajectory and lift coefficient time history at $U r=7.4$; (f) Motion trajectory and lift coefficient in frequency domain at $U r=7.4$; (g) Motion trajectory and lift coefficient time history at $U r=10.0$; (h) Motion trajectory and lift coefficient in frequency domain at $U r=10.0$; (i) Motion trajectory and lift coefficient time history at $U r=13.6$; (j) Motion trajectory and lift coefficient in frequency domain at $U r=13.6$.

Fig. 11 Time history of lift coefficient and transverse motion for chamfered corner design. (Both in time and frequency domain). (a) Motion trajectory and lift coefficient time history at $U r=4.7$; (b) Motion trajectory and lift coefficient in frequency domain at $U r=4.7$; (c) Motion trajectory and lift coefficient time history at $U r=6.2$; (d) Motion trajectory and lift coefficient in frequency domain at $U r=6.2$; (e) Motion trajectory and lift coefficient time history at $U r=8.0$; (f) Motion trajectory and lift coefficient in frequency domain at $U r=8.0 ;(\mathrm{g})$ Motion trajectory and lift coefficient time history at $U r=10.8$; (h) Motion trajectory and lift coefficient in frequency domain at $U r=10.8$; (i) Motion trajectory and lift coefficient time history at $U r=14.7 ;$ (j) Motion trajectory and lift coefficient in frequency domain at $U r=14.7 .29$ 
Fig. 12 Non-dimensional transverse response frequency $f y / f N$ and non-

800 dimensional vortex shedding frequency $f_{s} / f N(*$ is the secondary peak observed in the

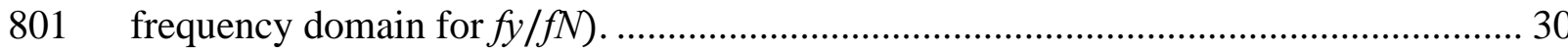

802 Fig. 13 Motion trajectories in the $X Y$ plane for the sharp corner design............ 31

803 Fig. 14 Motion trajectories in the $X Y$ plane for the rounded corner design. ...... 31

804 Fig. 15 Motion trajectories in the $X Y$ plane for the chamfered corner design.... 32

805 Fig. 16 Phase angle between lift coefficient and transverse amplitude. 33

806 Fig. 17 Root mean square lift coefficient (CLrms) and work done on each

807 member of the structure. (a) sharp corner design; (b) rounded corner design; (c)

808 chamfered corner design. 34

809 Fig. 18 Time series of the non-dimensional (a) transverse motion response

810 frequency and (b) vortex shedding frequency with the frequency energy contours based

811 on continuous wavelet transforms in the "post lock-in" region for a sharp corner design.

812 36

814 frequency and (b) vortex shedding frequency with the frequency energy contours based

815 on continuous wavelet transforms in the "post lock-in" region for a rounded corner

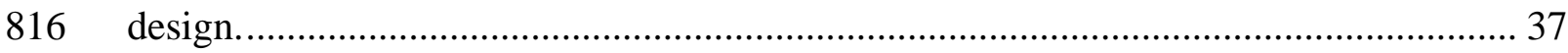

817 Fig. 20 Time series of the non-dimensional (a) transverse motion response

818 frequency and (b) vortex shedding frequency with the frequency energy contours based

819 on continuous wavelet transforms in the "post lock-in" region for a chamfered corner

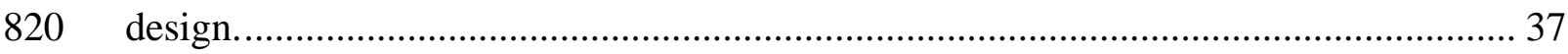


Fig. 21 Instantaneous vorticity contours and streamline for different corner

822 designs. (a) sharp corner design; (b) rounded corner design; (c) chamfered corner

823 design; (d) a local zoom vorticity contour in (c)

824 Fig. 22 Isometric view representation of $Q$-criterion of the three different corner

825 design covered by the non-dimensional velocity contours. (a) sharp corner design; (b)

826 rounded corner design; (c) chamfered corner design. 40

827 


\section{List of Tables}

829

Table 1 Principle dimensions of the model semi-submersible (with a scale ratio

830 of $1: 64)$ 11

831

Table 2 Validation of the natural periods of the motions in calm water. 17

832

Table 3 Natural periods of the motions in calm water. 19

833 
A)

B)

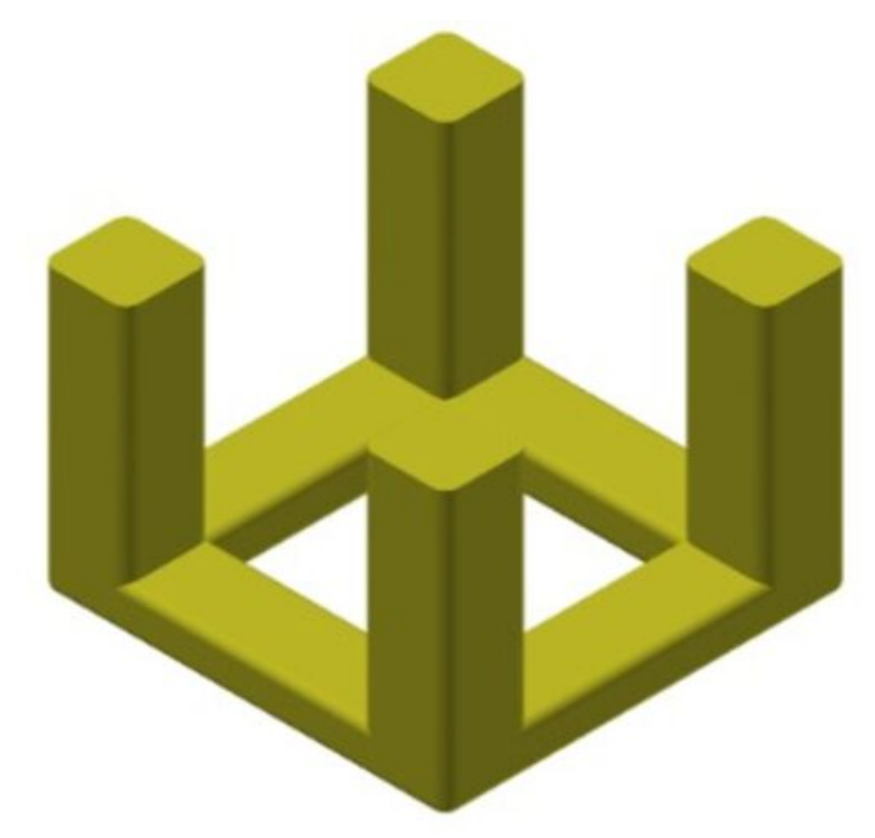

C)

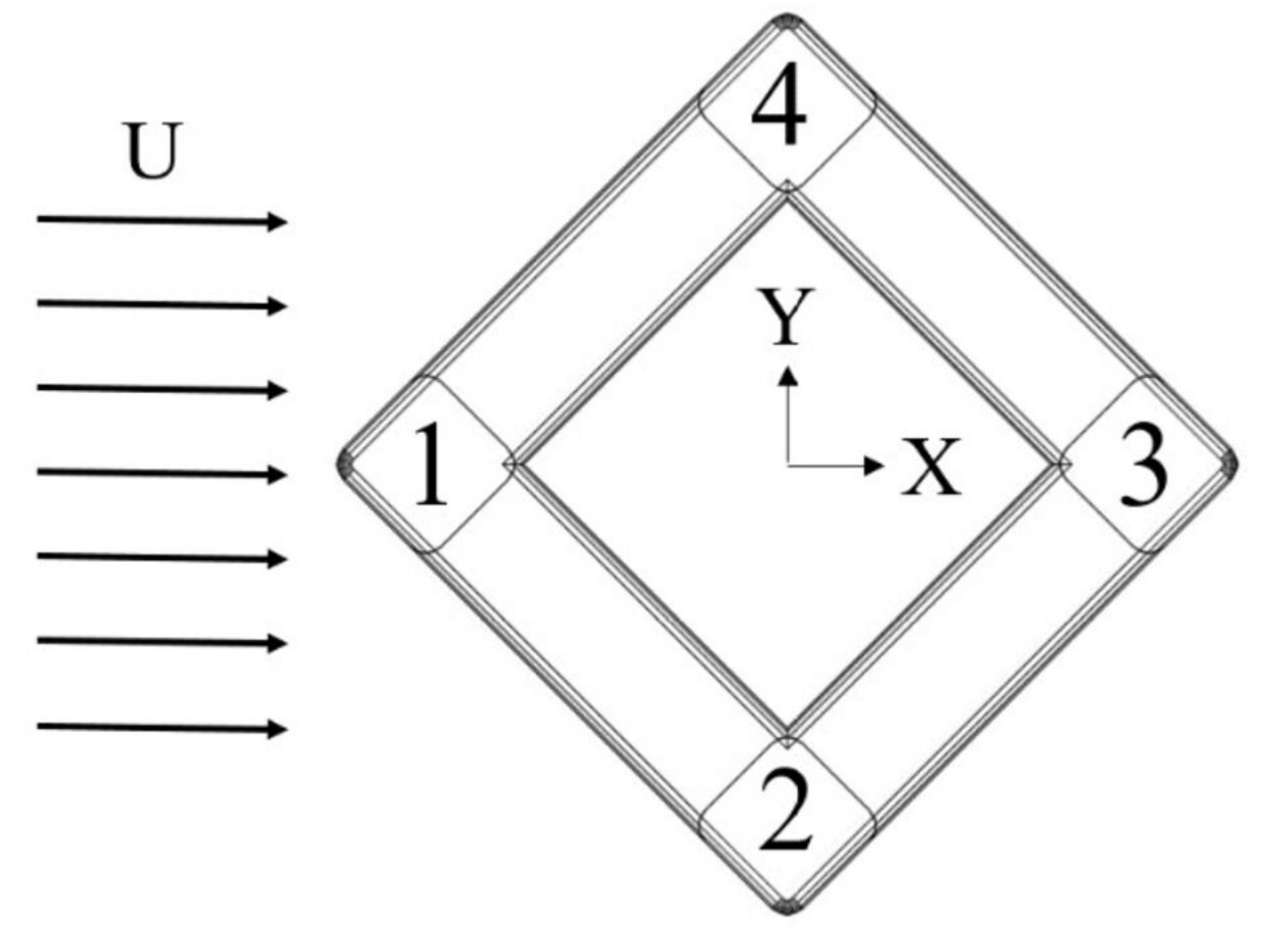




\section{Transverse}

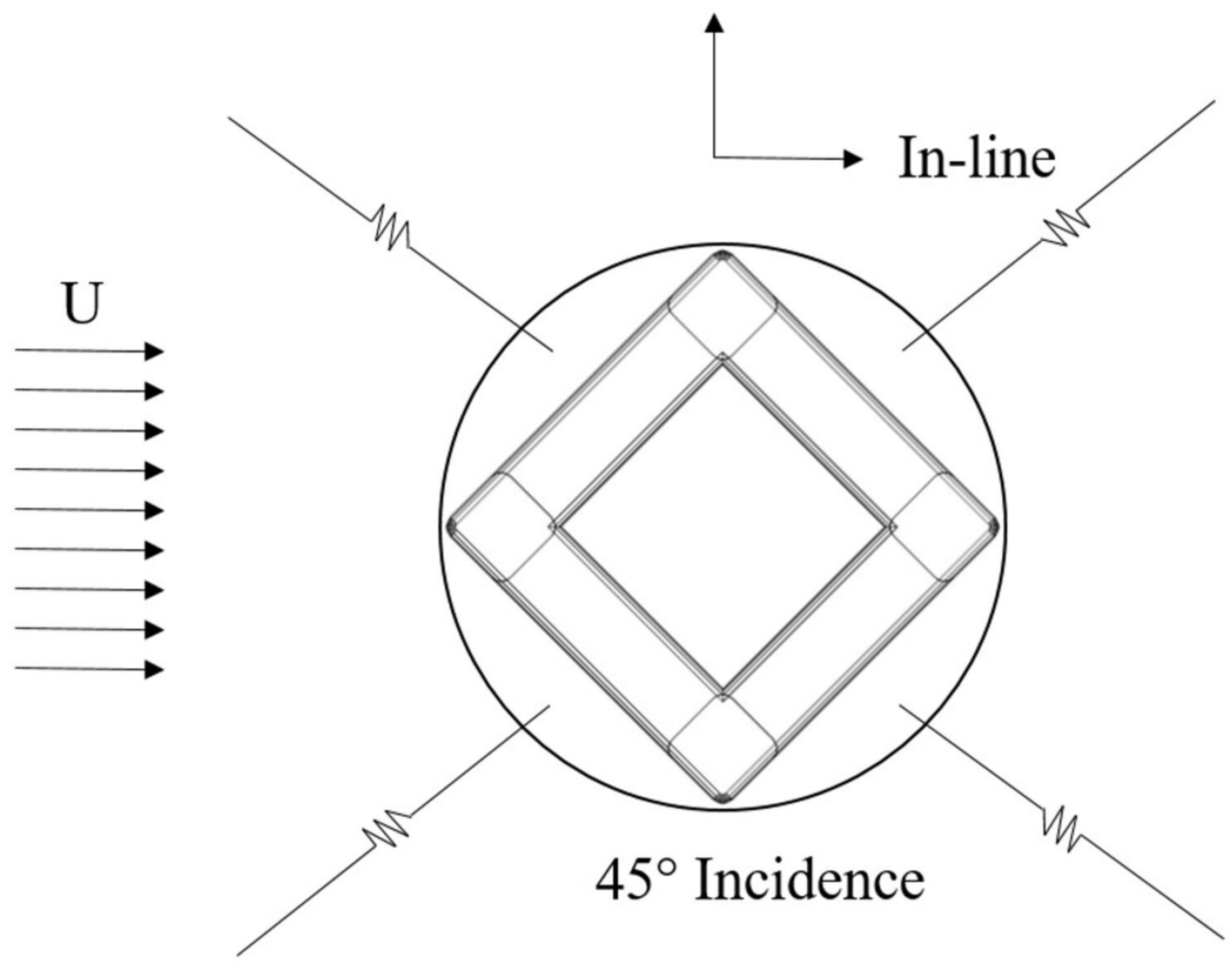




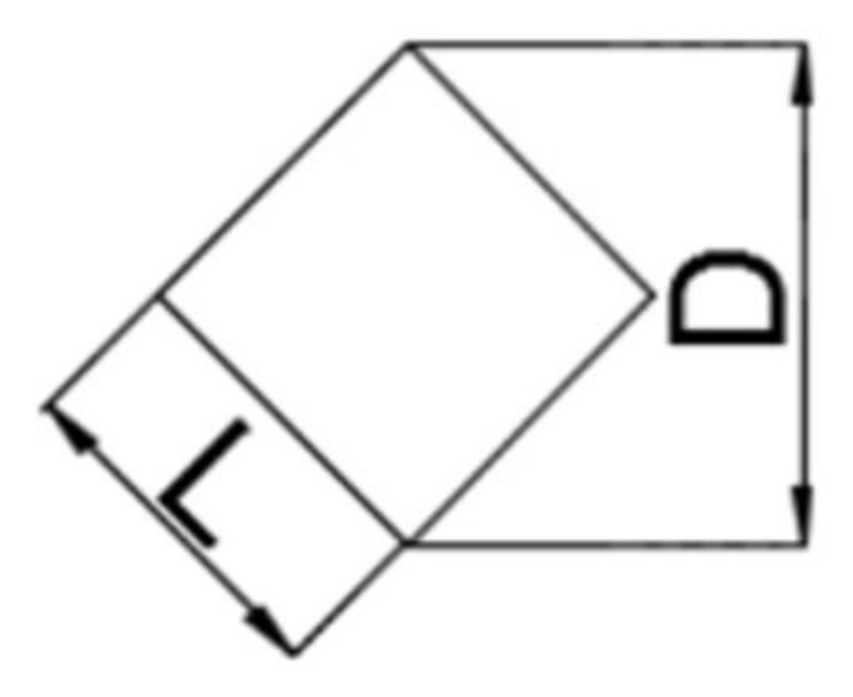

Sharp corners

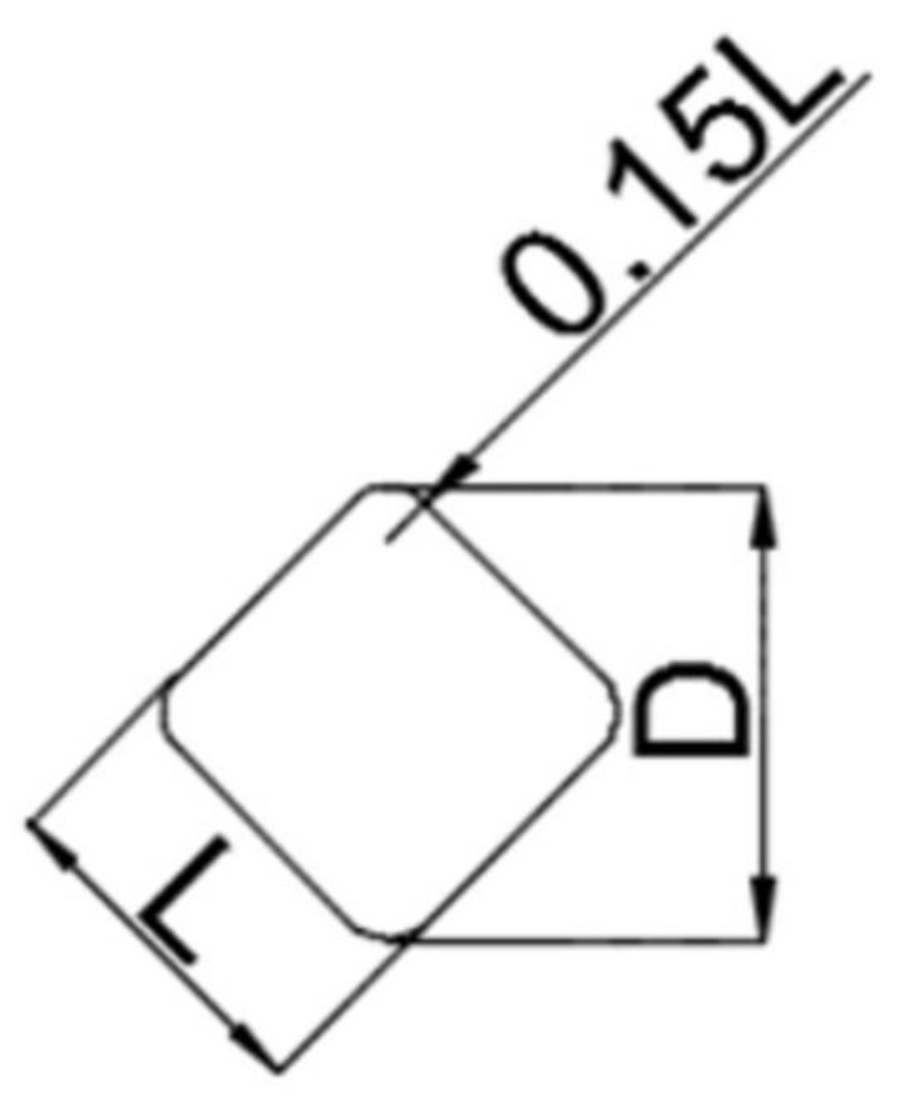

Rounded corners

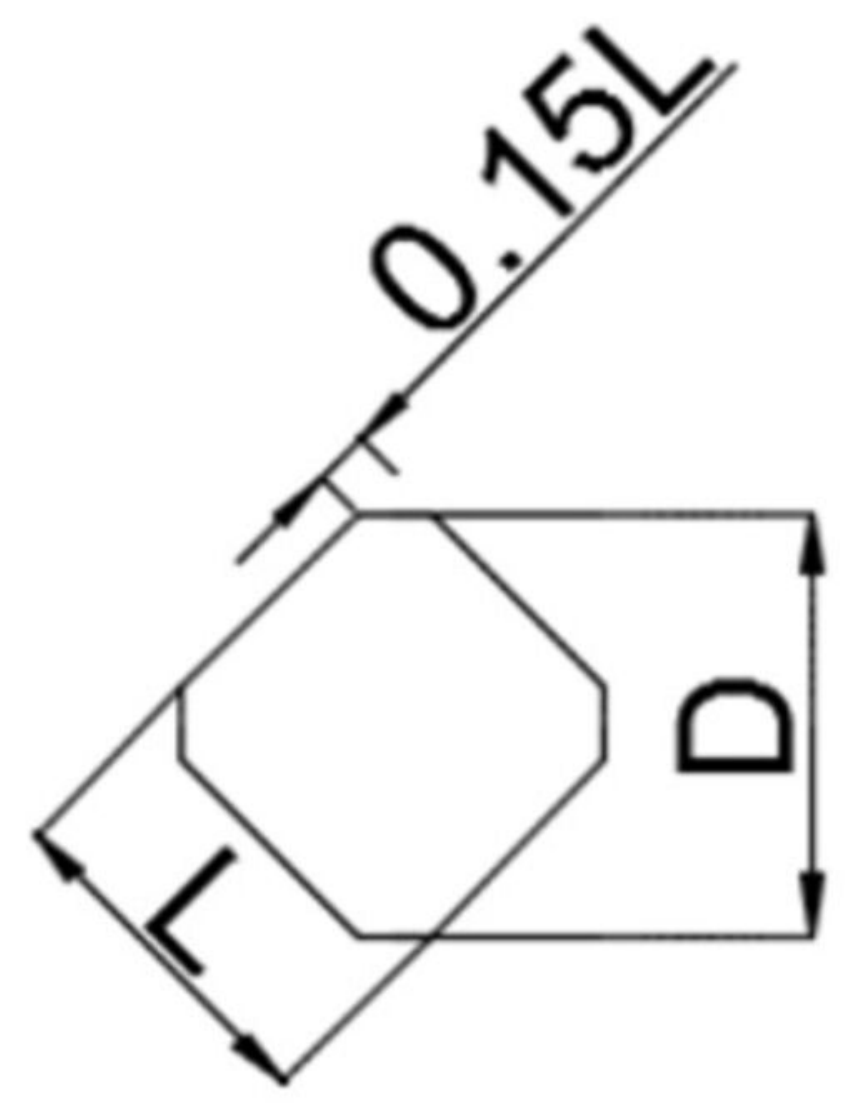

Chamfered corners 


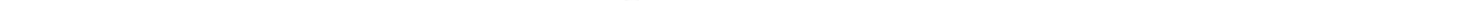




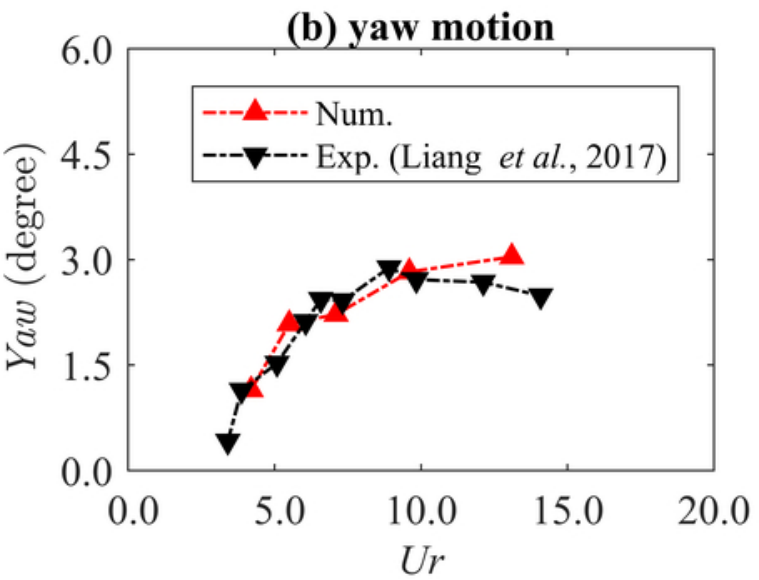




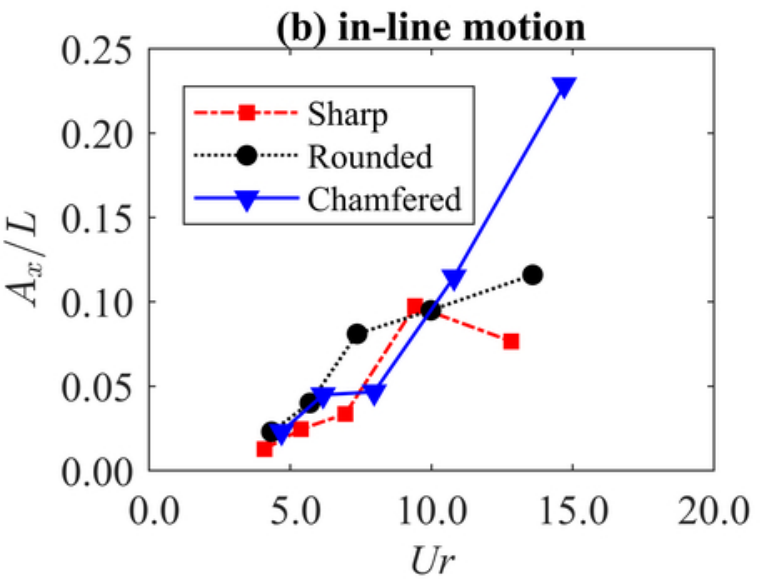




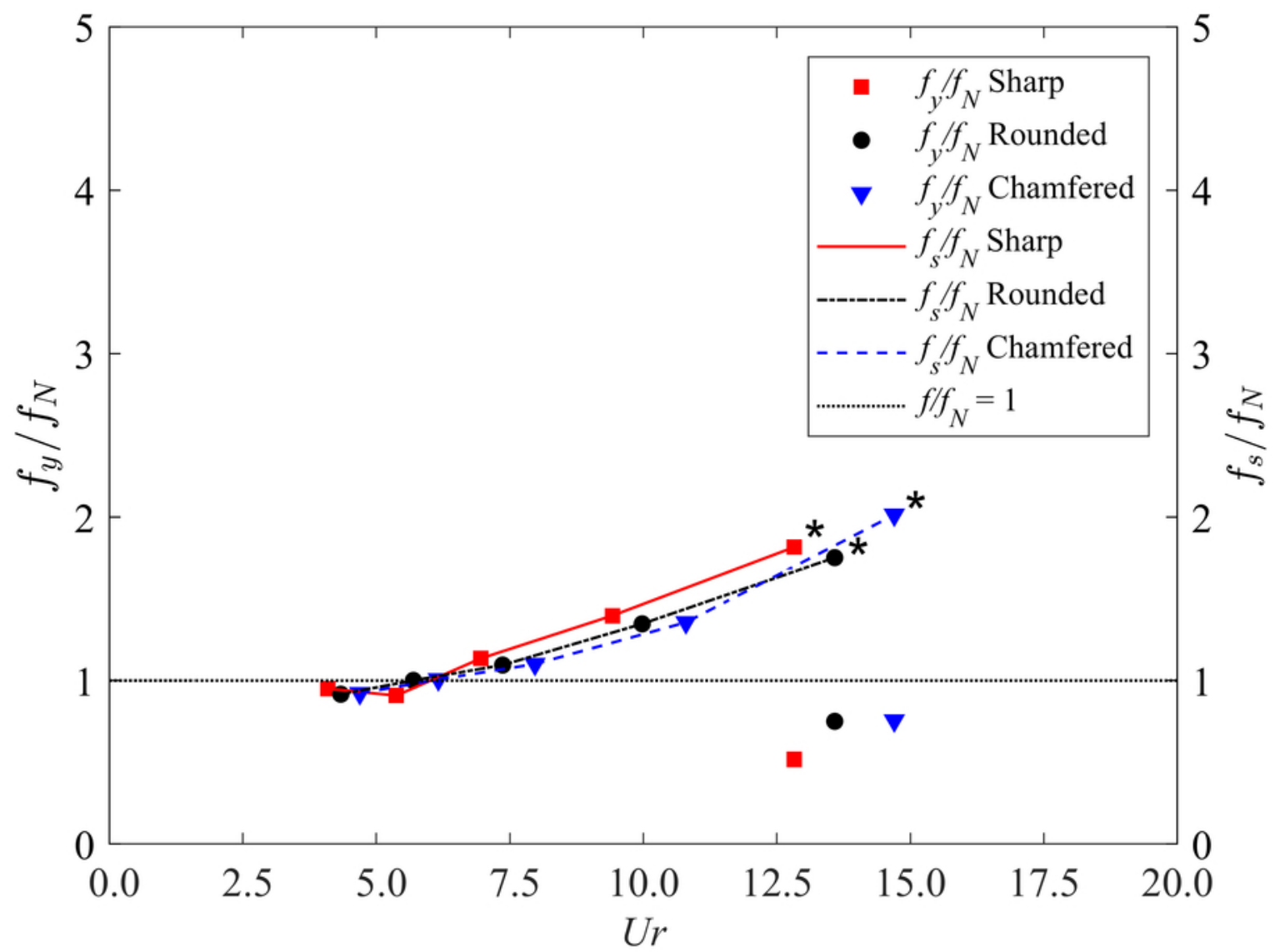




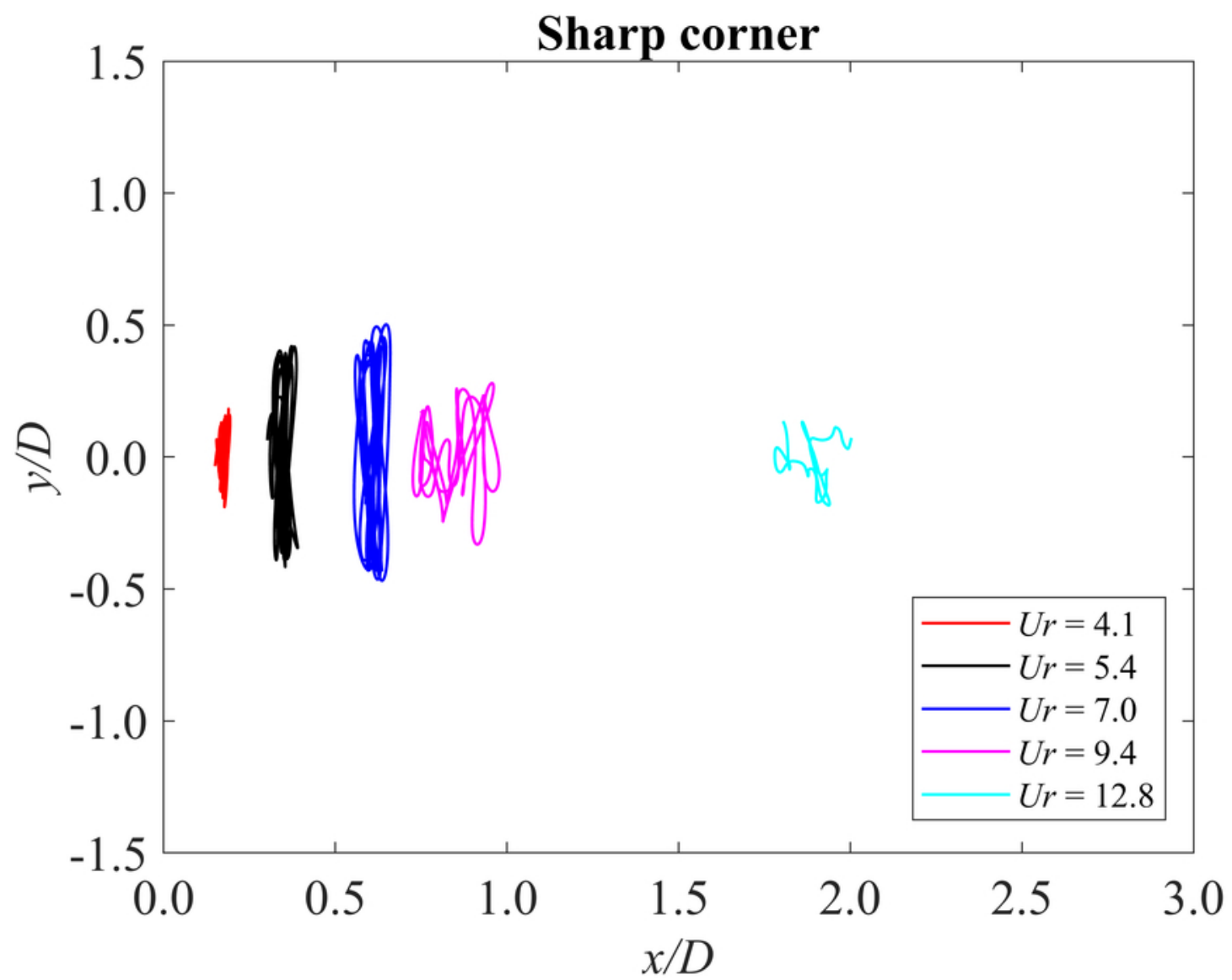




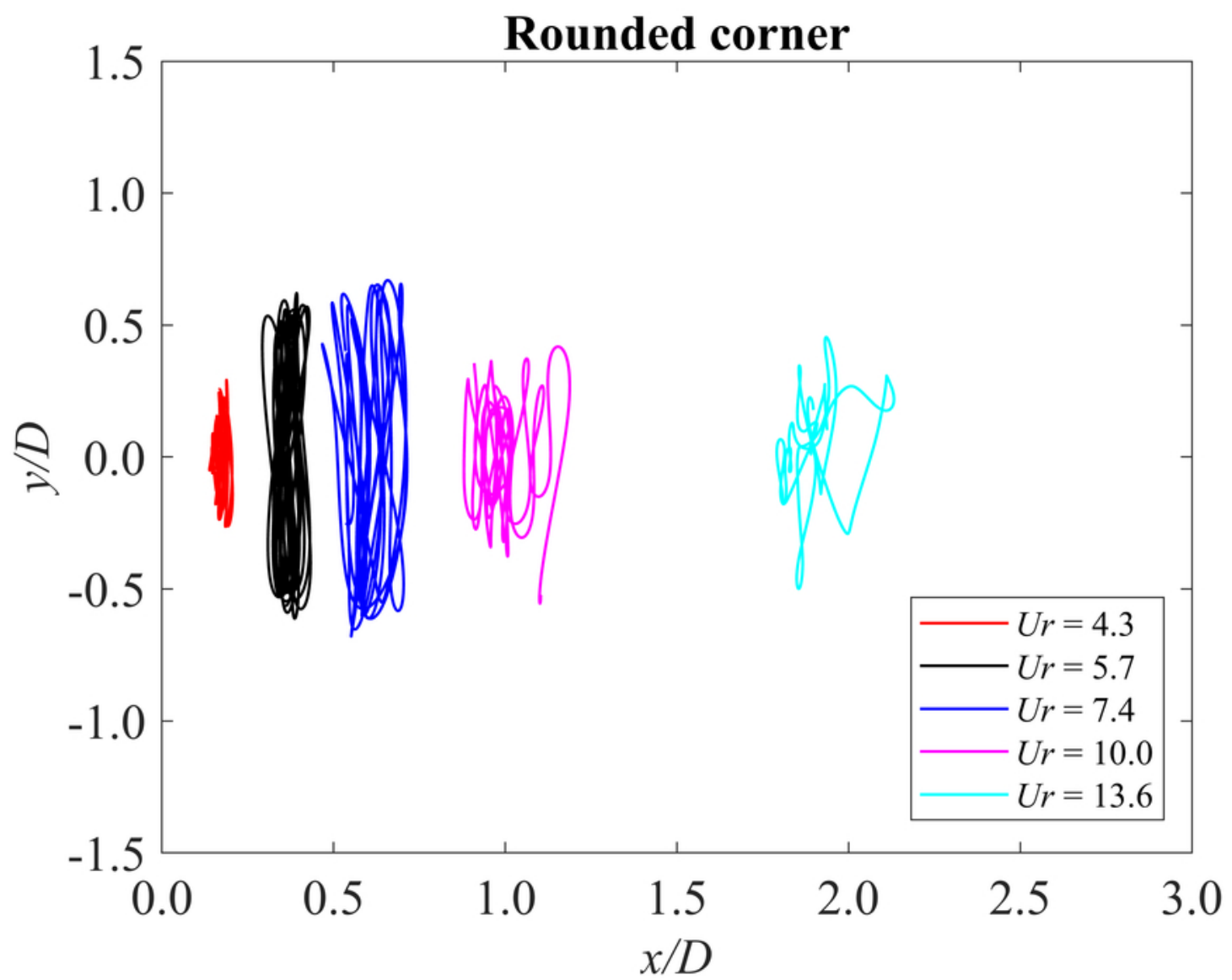




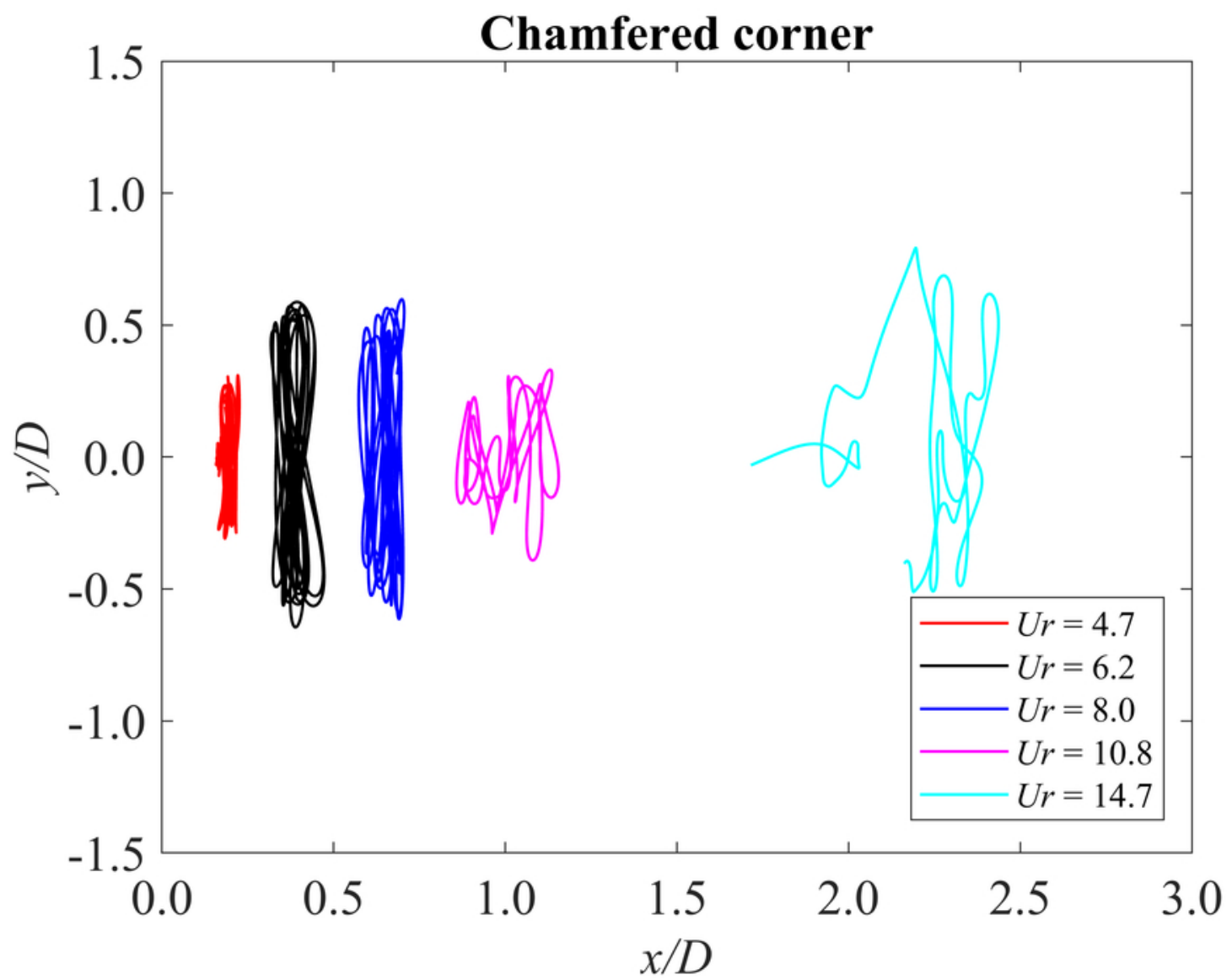




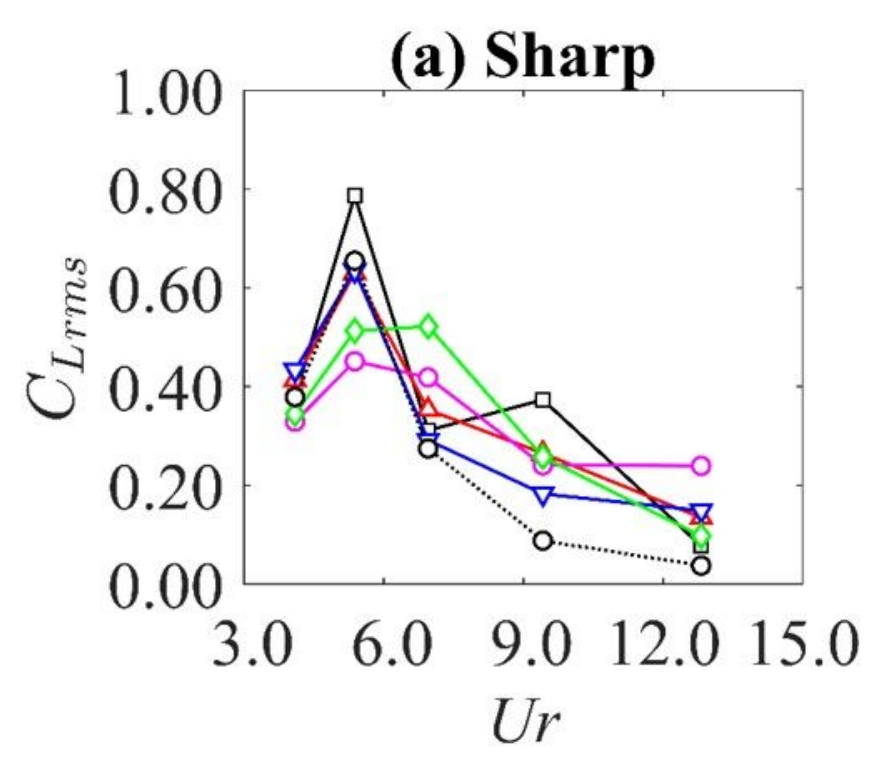

\section{(b) Rounded}

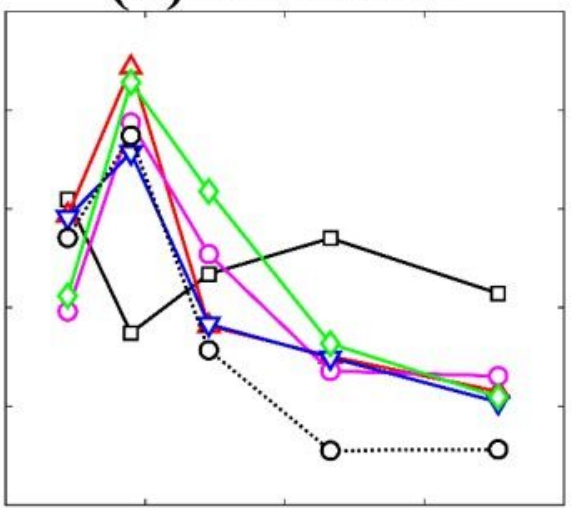

$\begin{array}{lllll}3.0 & 6.0 & 9.0 & 12.0 & 15.0\end{array}$ Ur

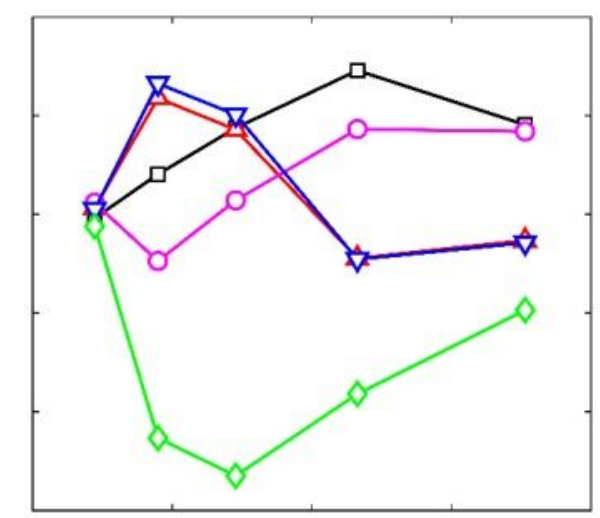

$\begin{array}{lllll}3.0 & 6.0 & 9.0 & 12.0 & 15.0 \\ & & U r & \end{array}$ (c) Chamfered
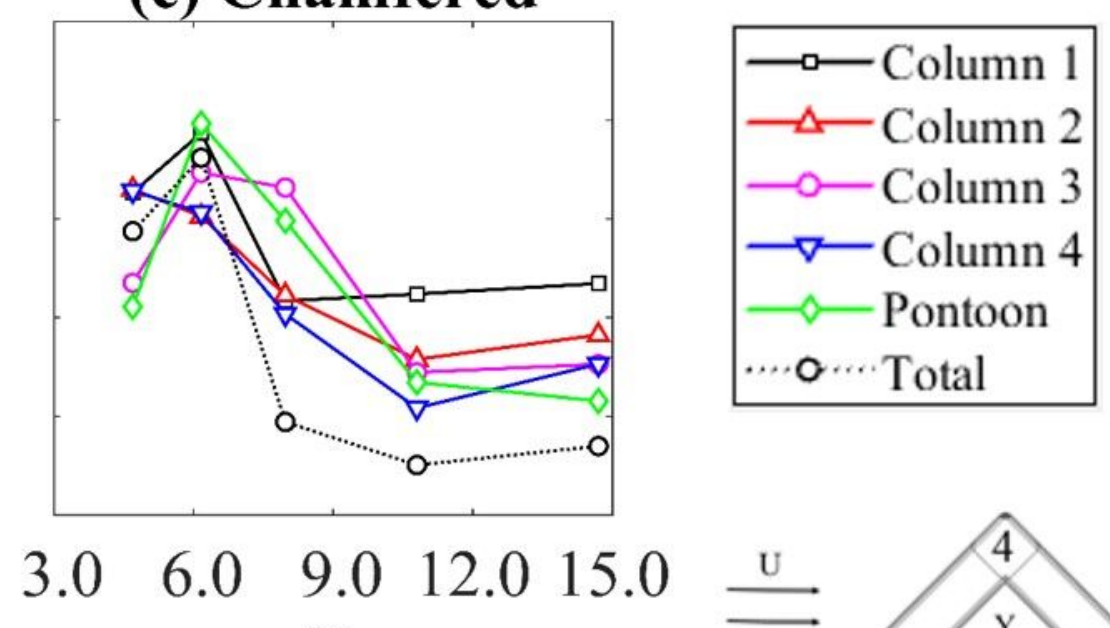

Ur

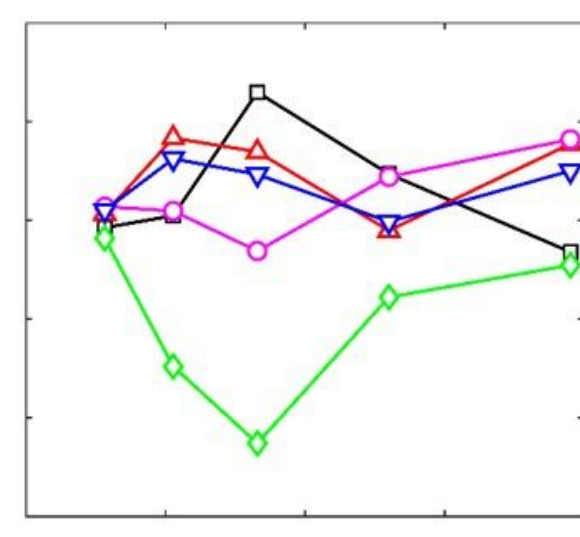

$\begin{array}{lllll}3.0 & 6.0 & 9.0 & 12.0 & 15.0 \\ & U r\end{array}$

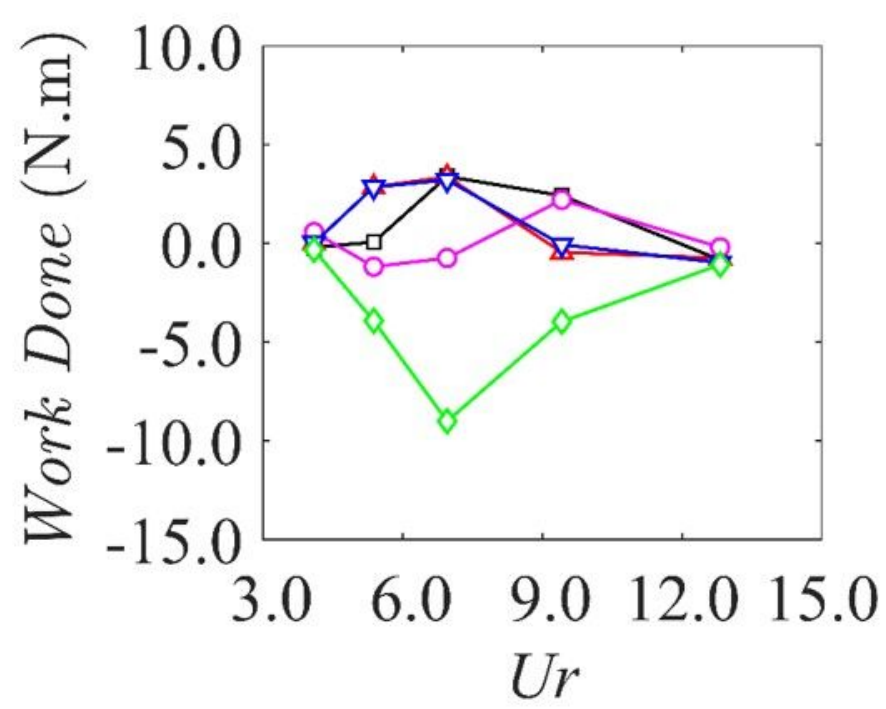




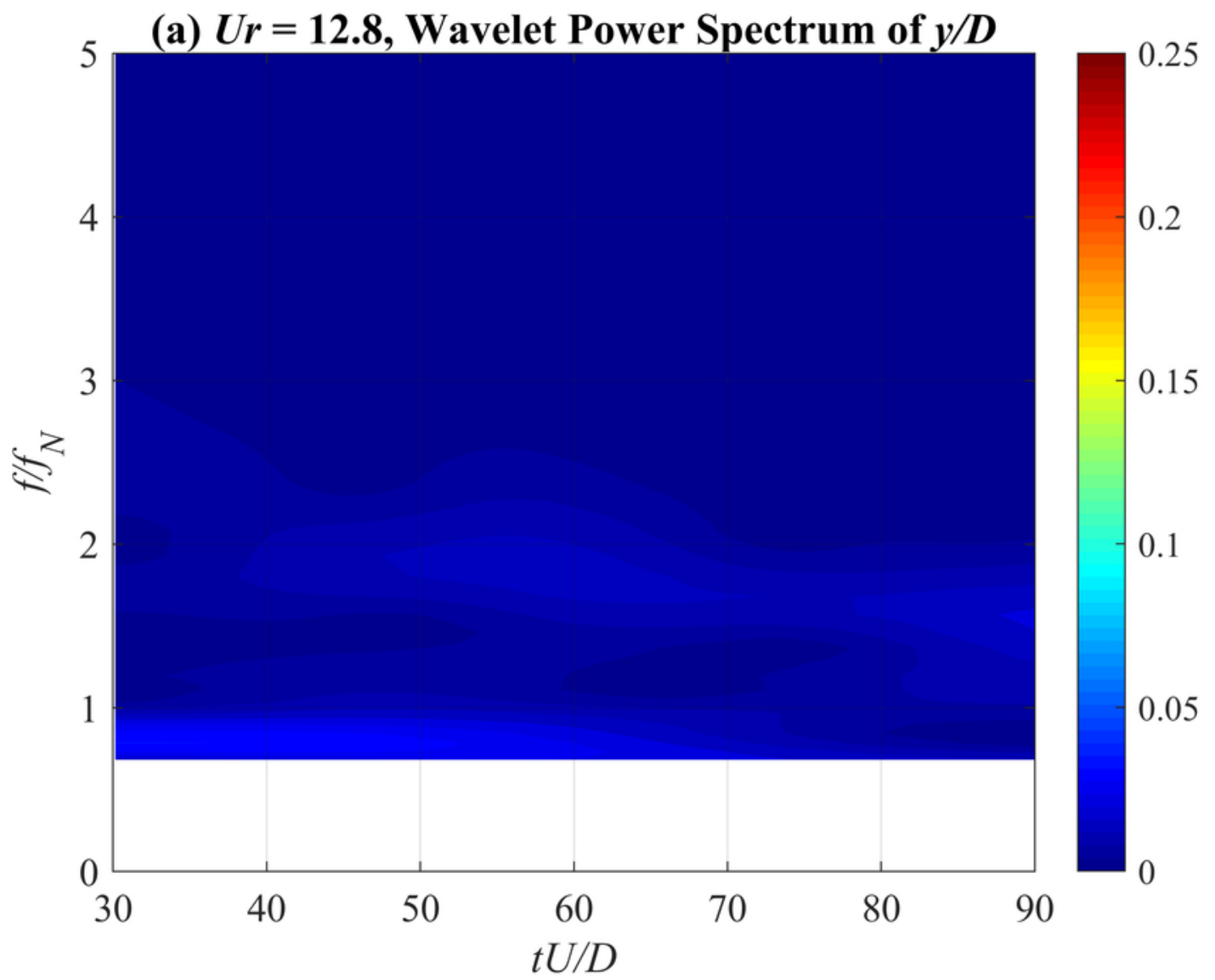


(b) $U r=12.8$, Wavelet Power Spectrum of $C_{L}$

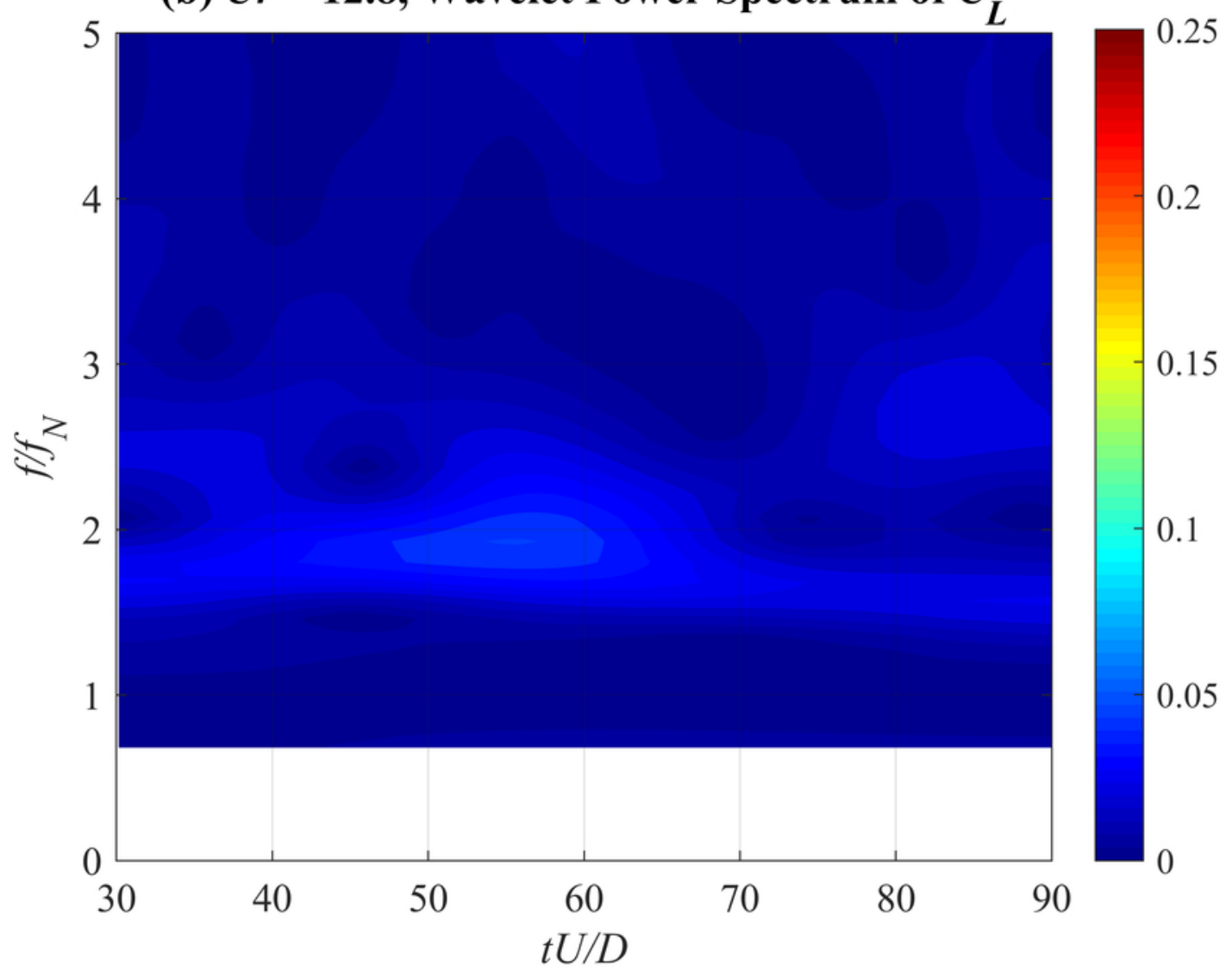




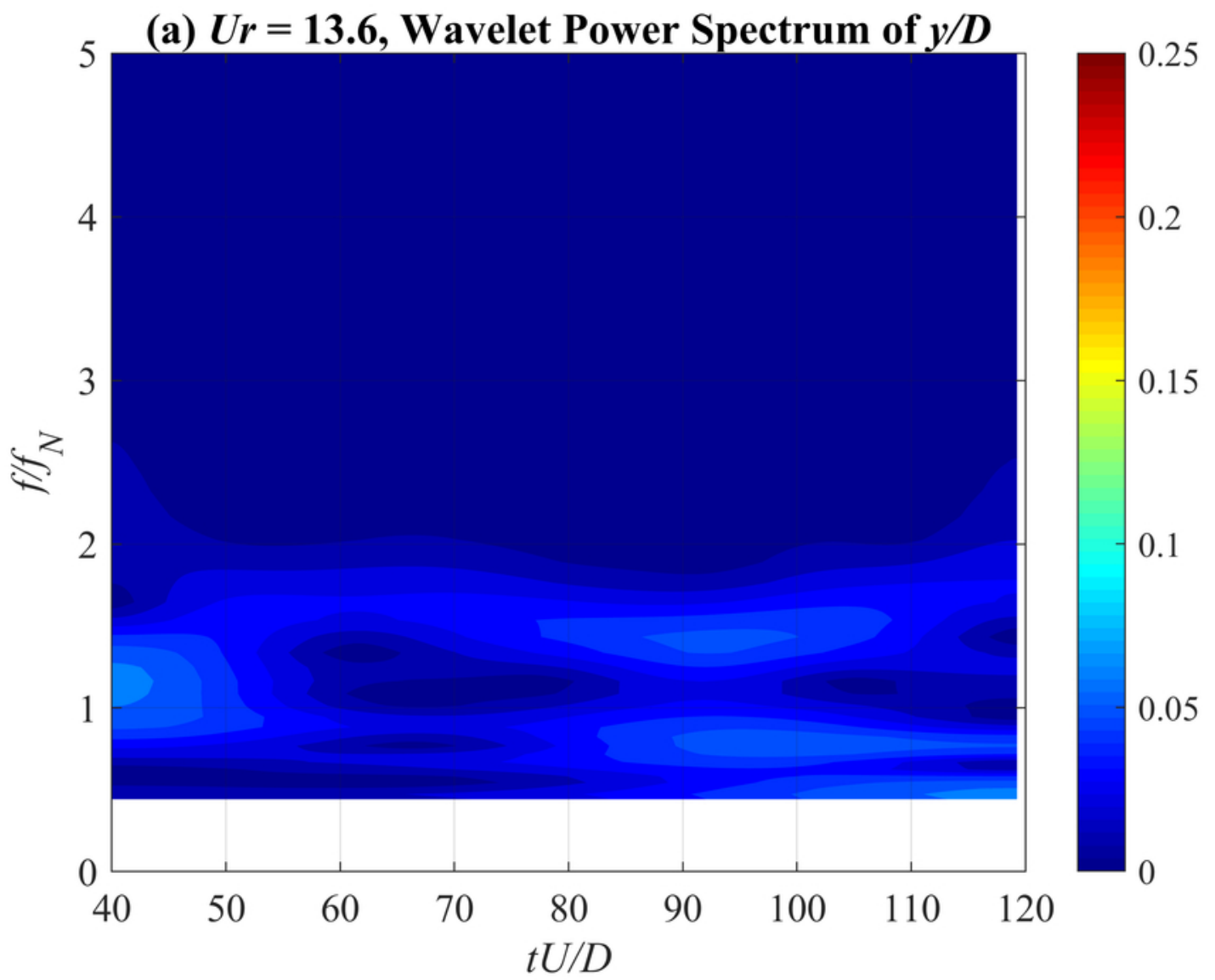


(b) $U r=$ 13.6, Wavelet Power Spectrum of $C_{L}$

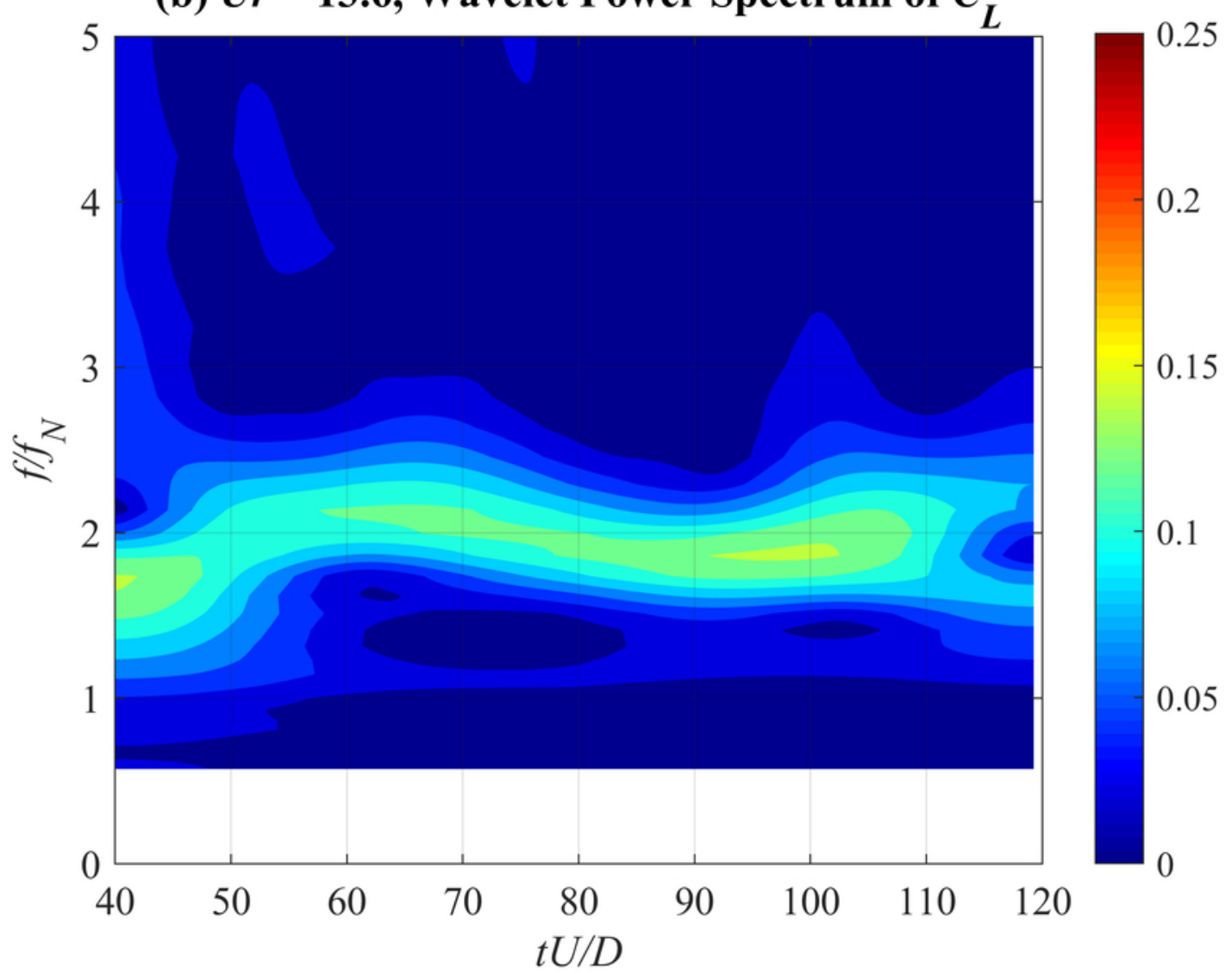




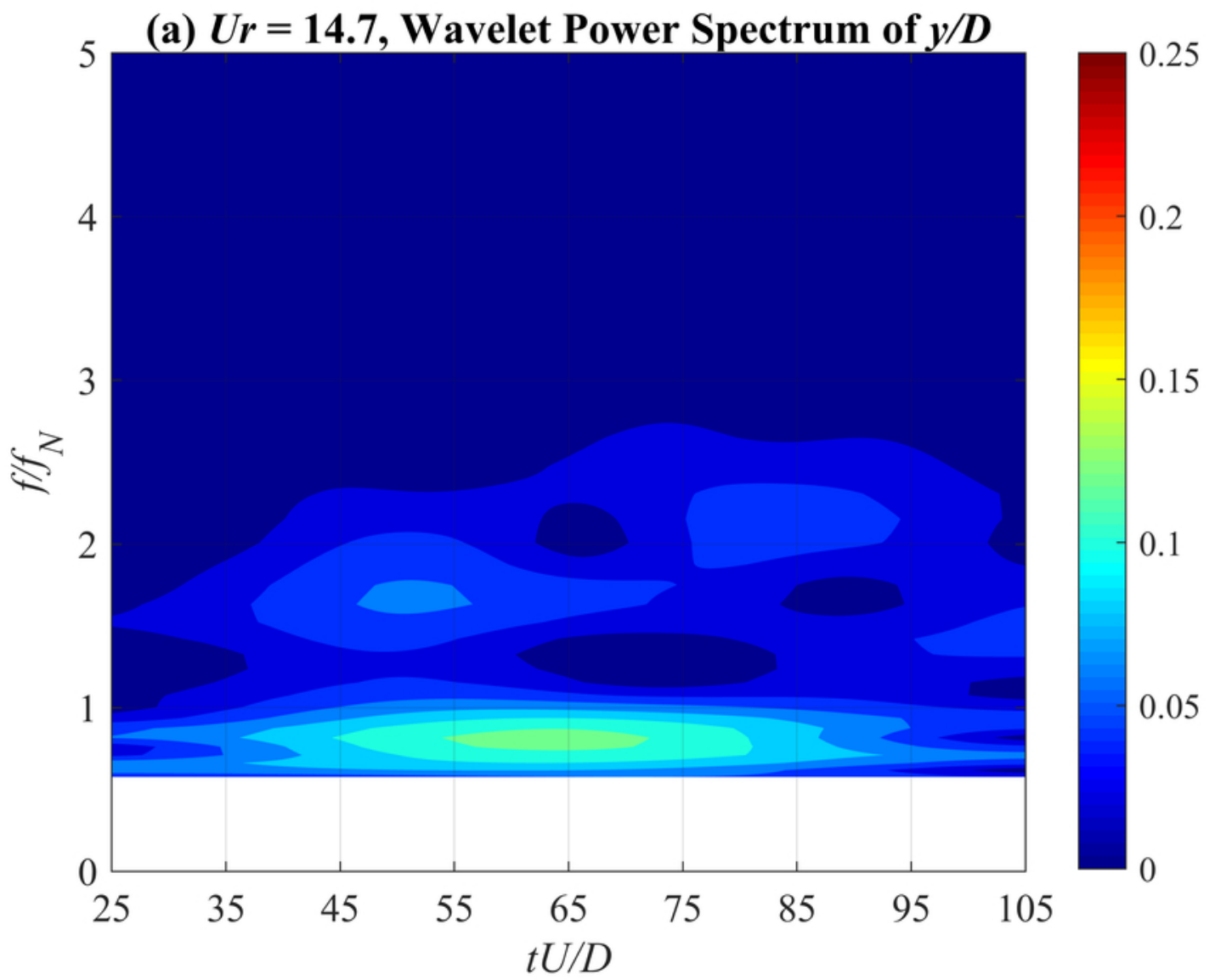


(b) $U r=14.7$, Wavelet Power Spectrum of $C_{L}$

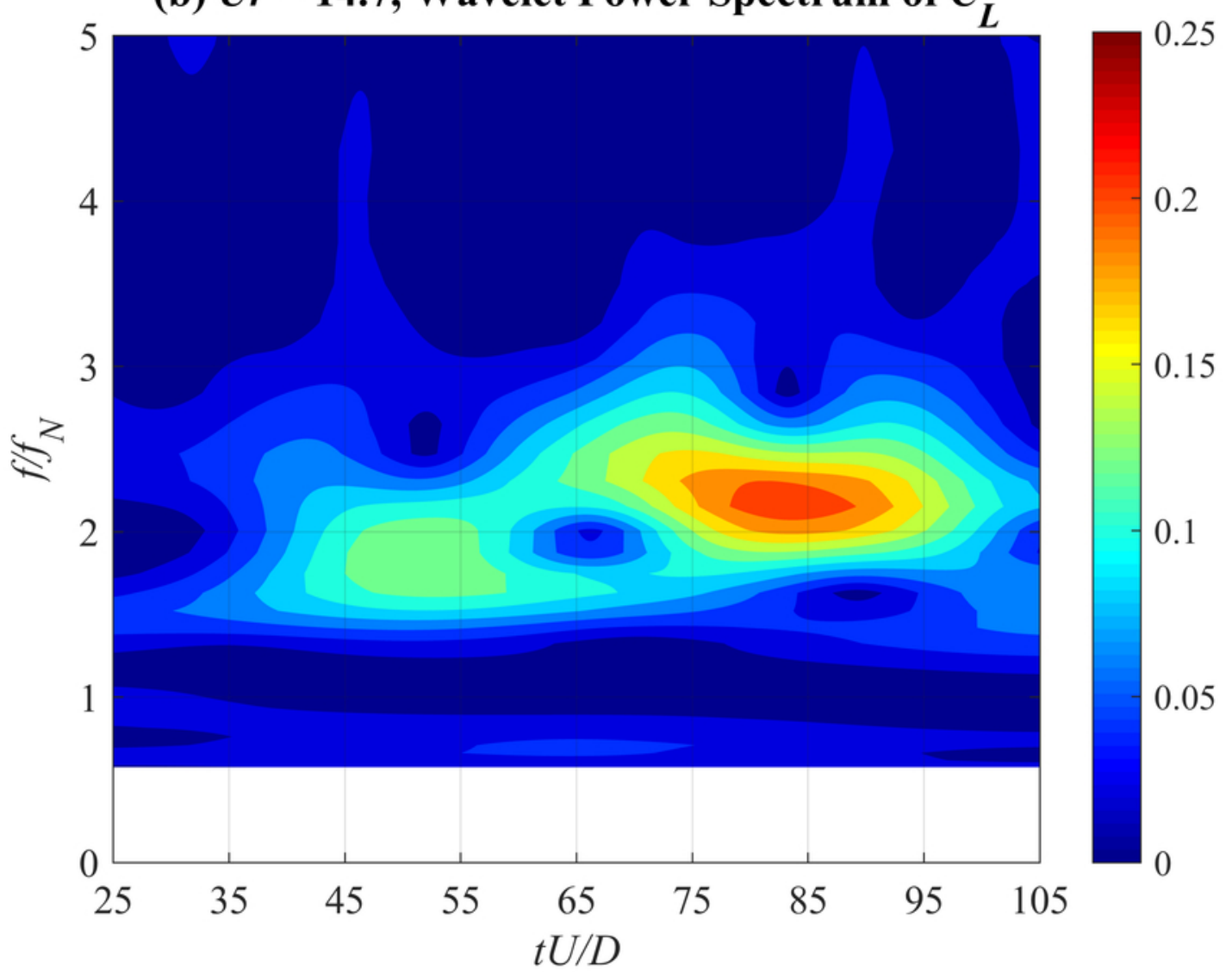


(a) Sharp corner

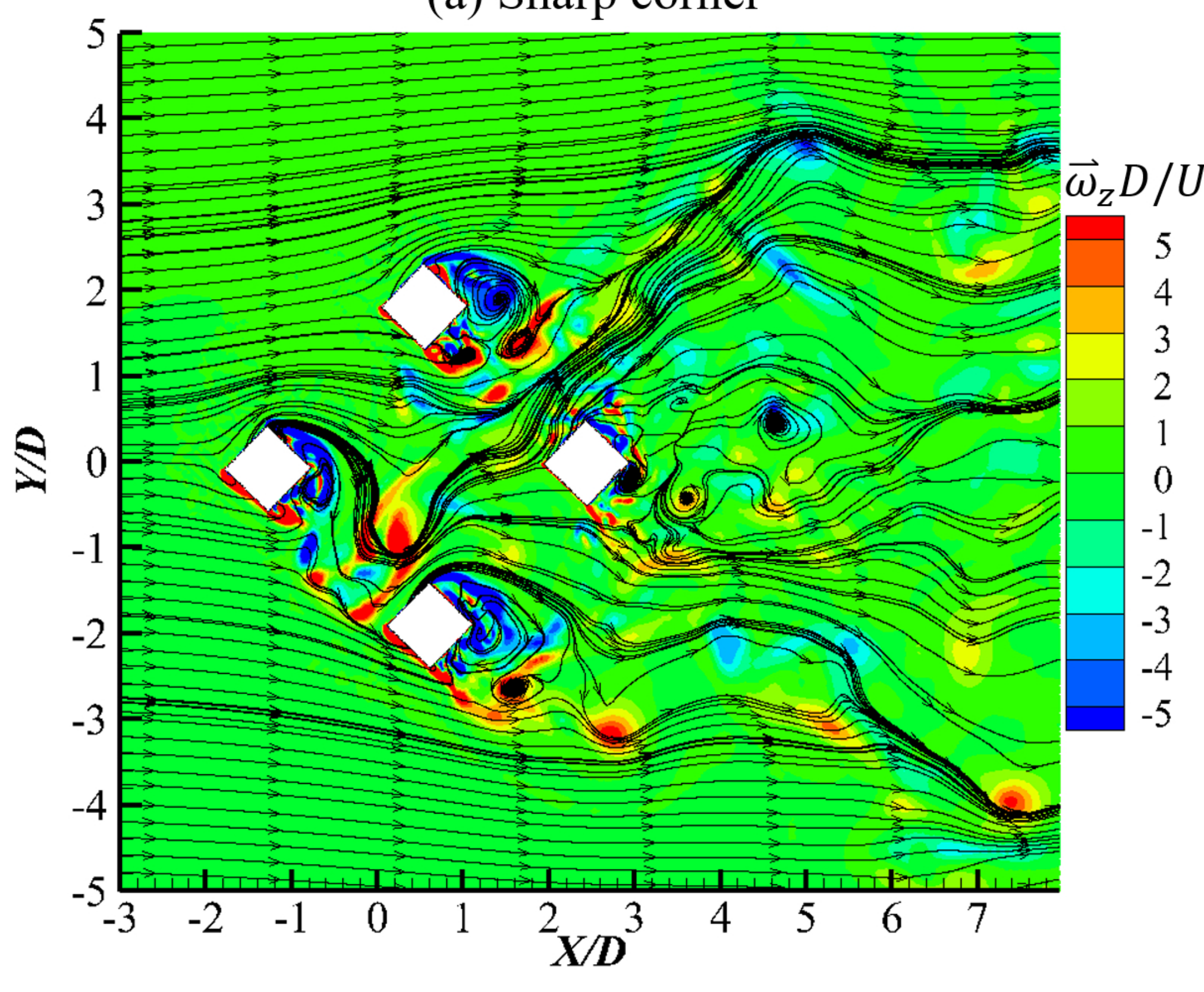

(c) Chamfered corner

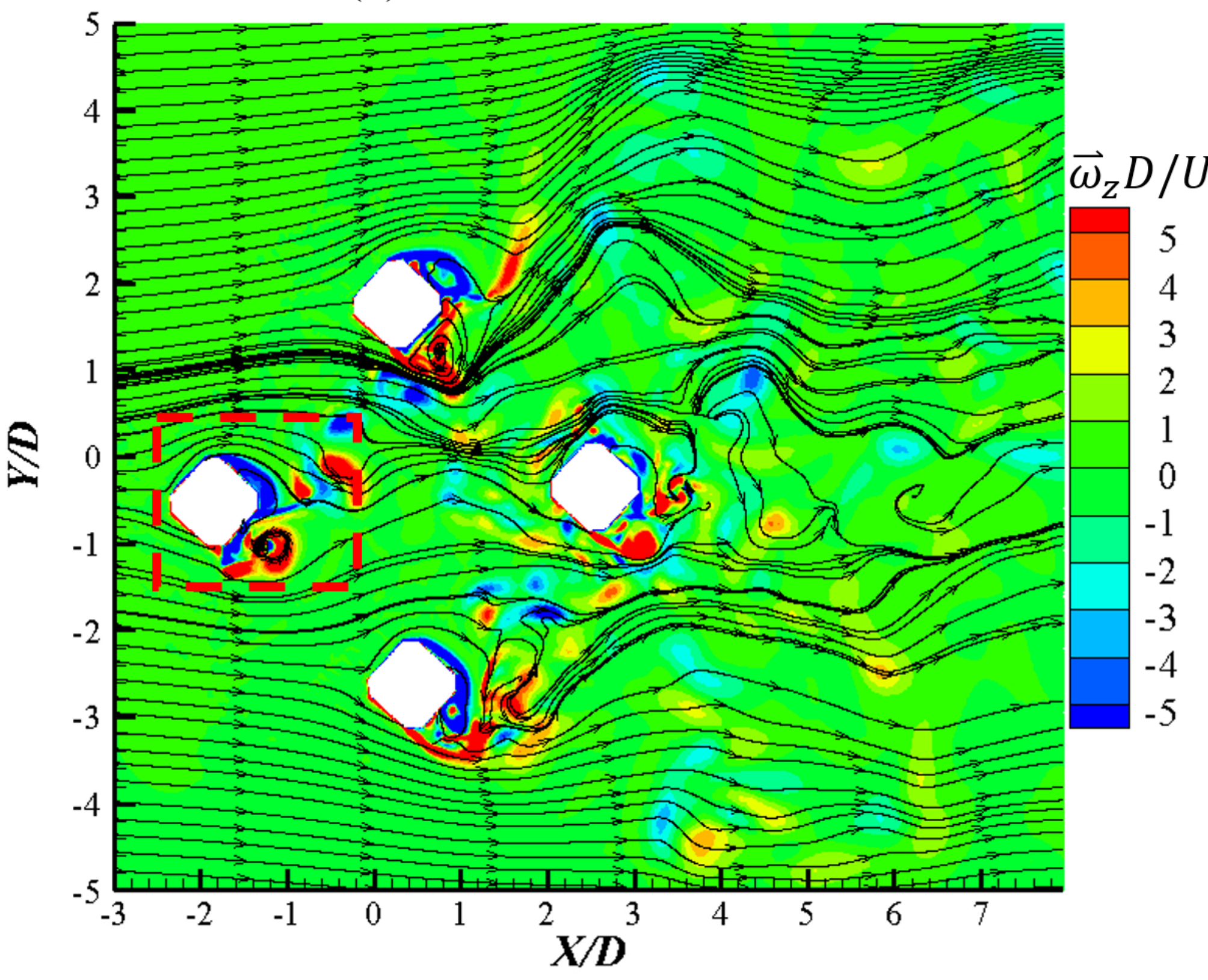

(b) Rounded corner

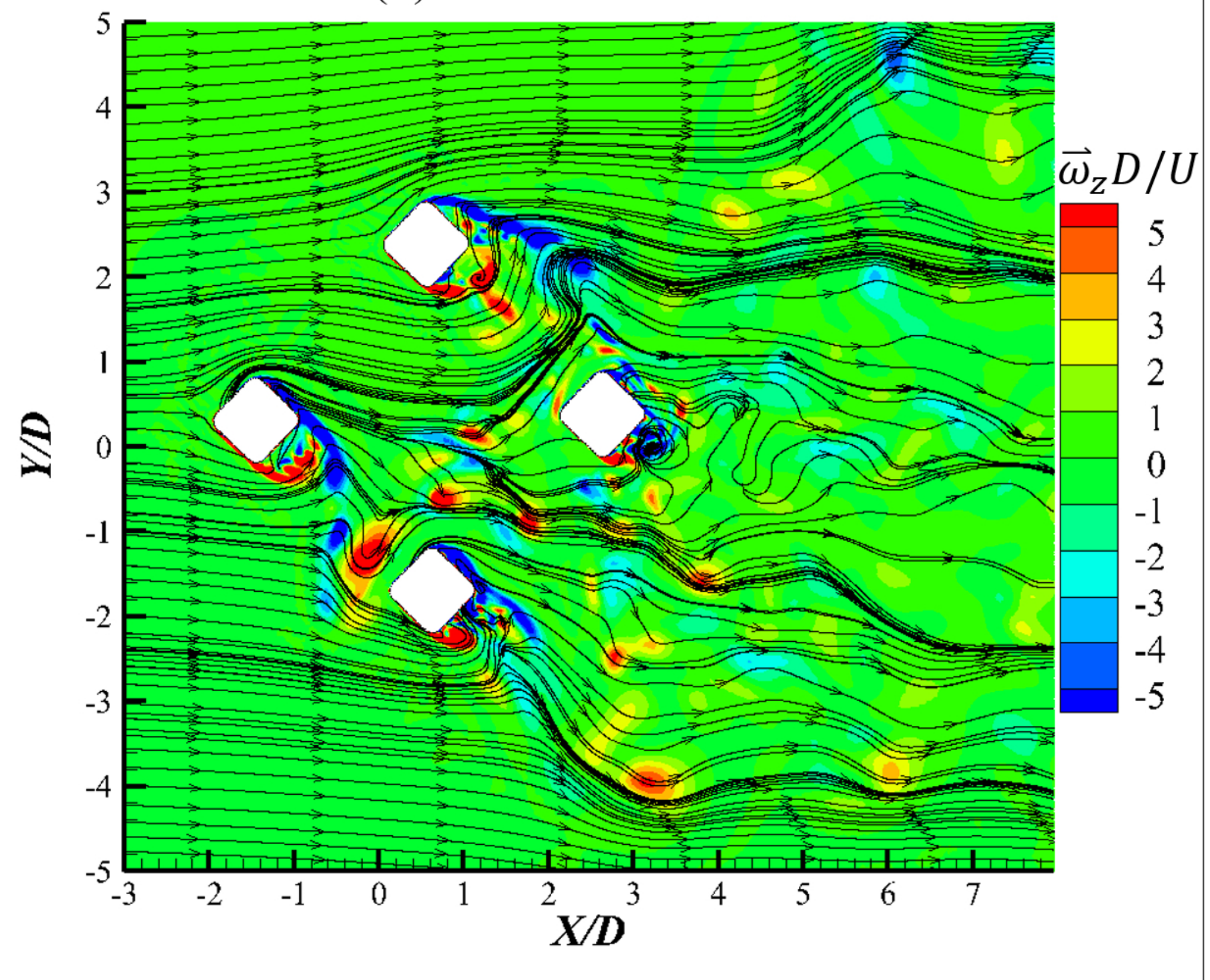

(d) Local zoom in sub figure (c)

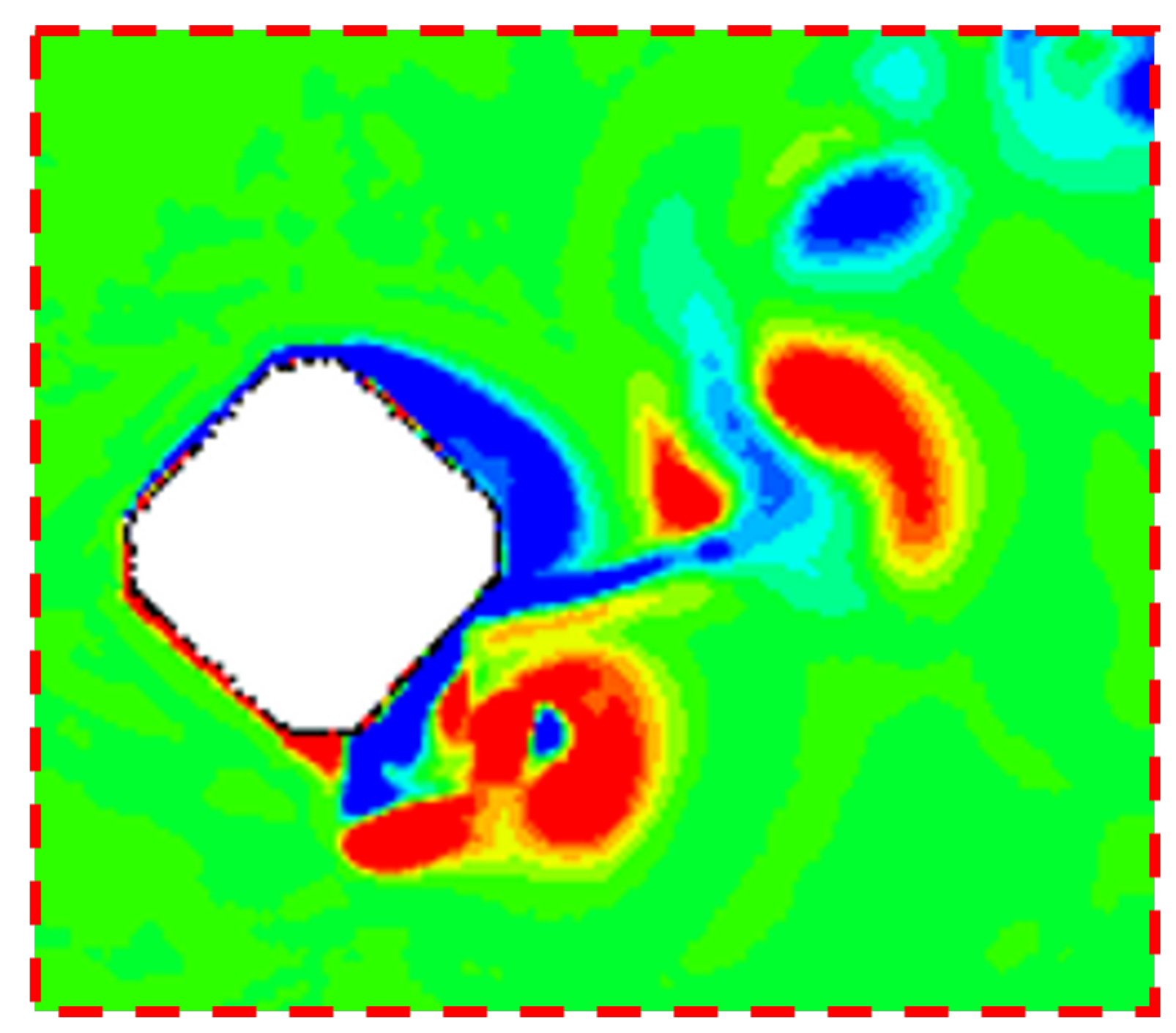


(a) Sharp corner

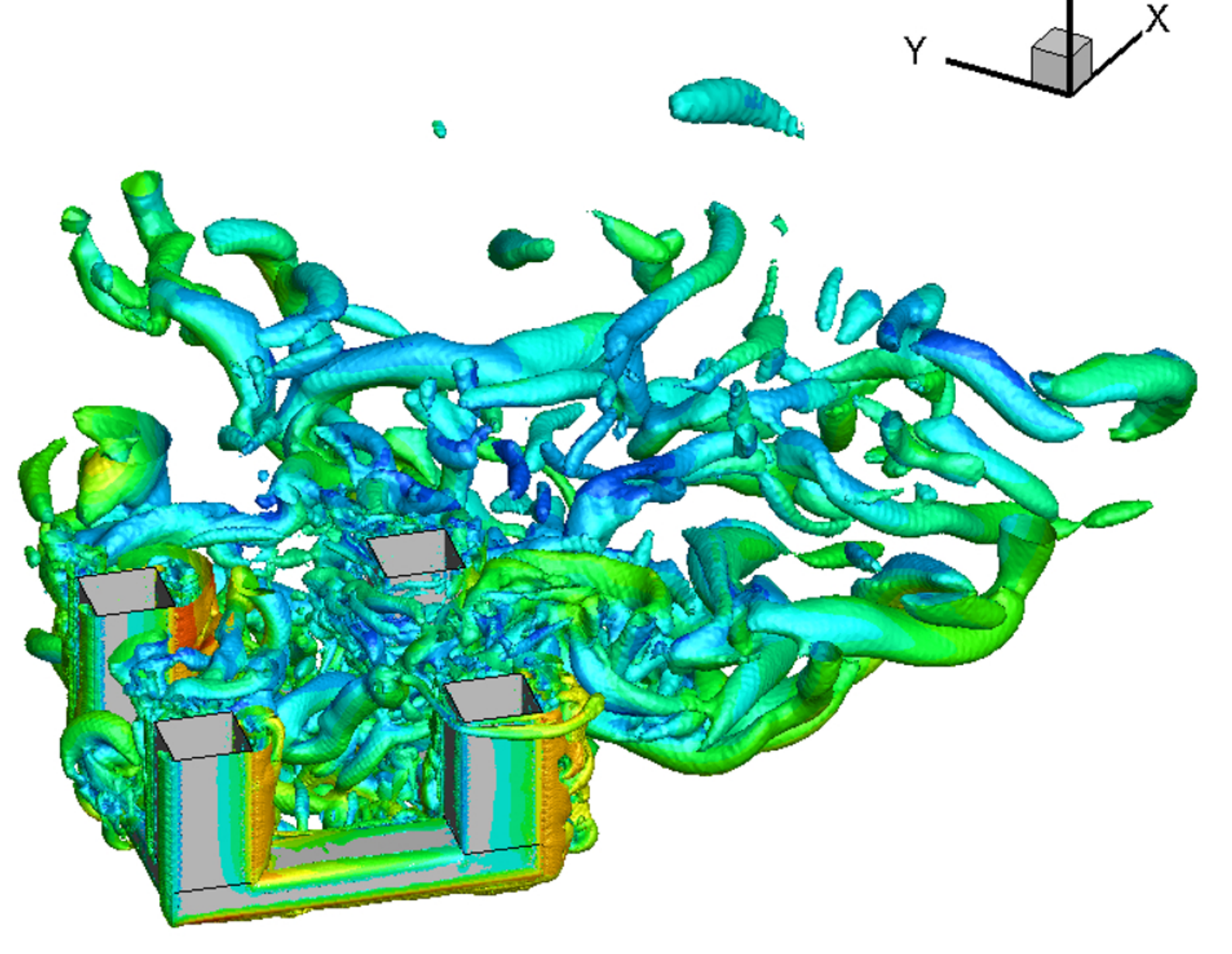

$U_{m a g} / U$

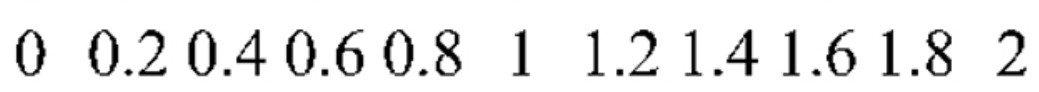

(b) Rounded corner
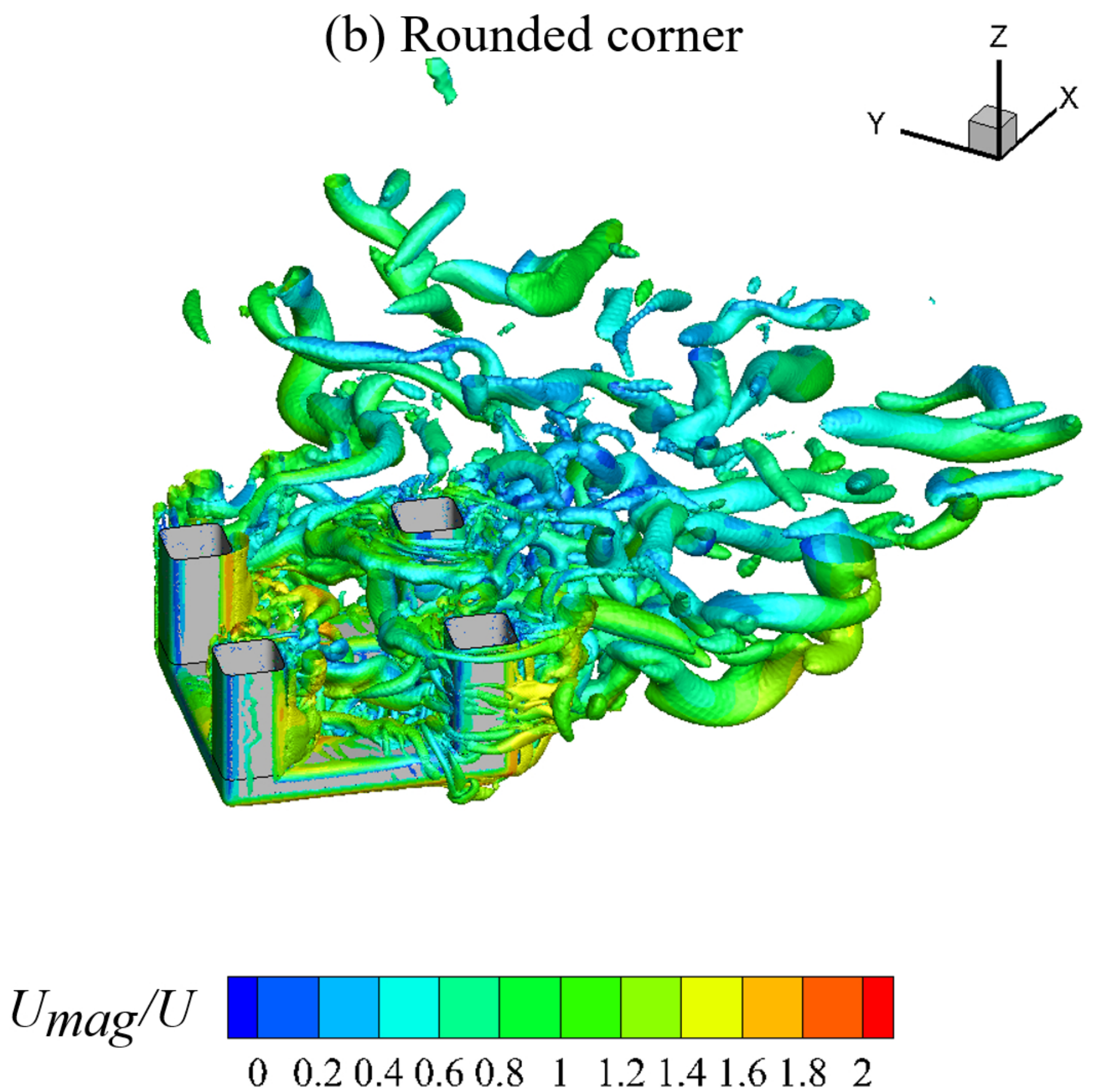

$\begin{array}{lllllllll}0 & 0.2 & 0.4 & 0.60 .8 & 1 & 1.2 & 1.41 .61 .8 & 2\end{array}$ (c) Chamfered corner
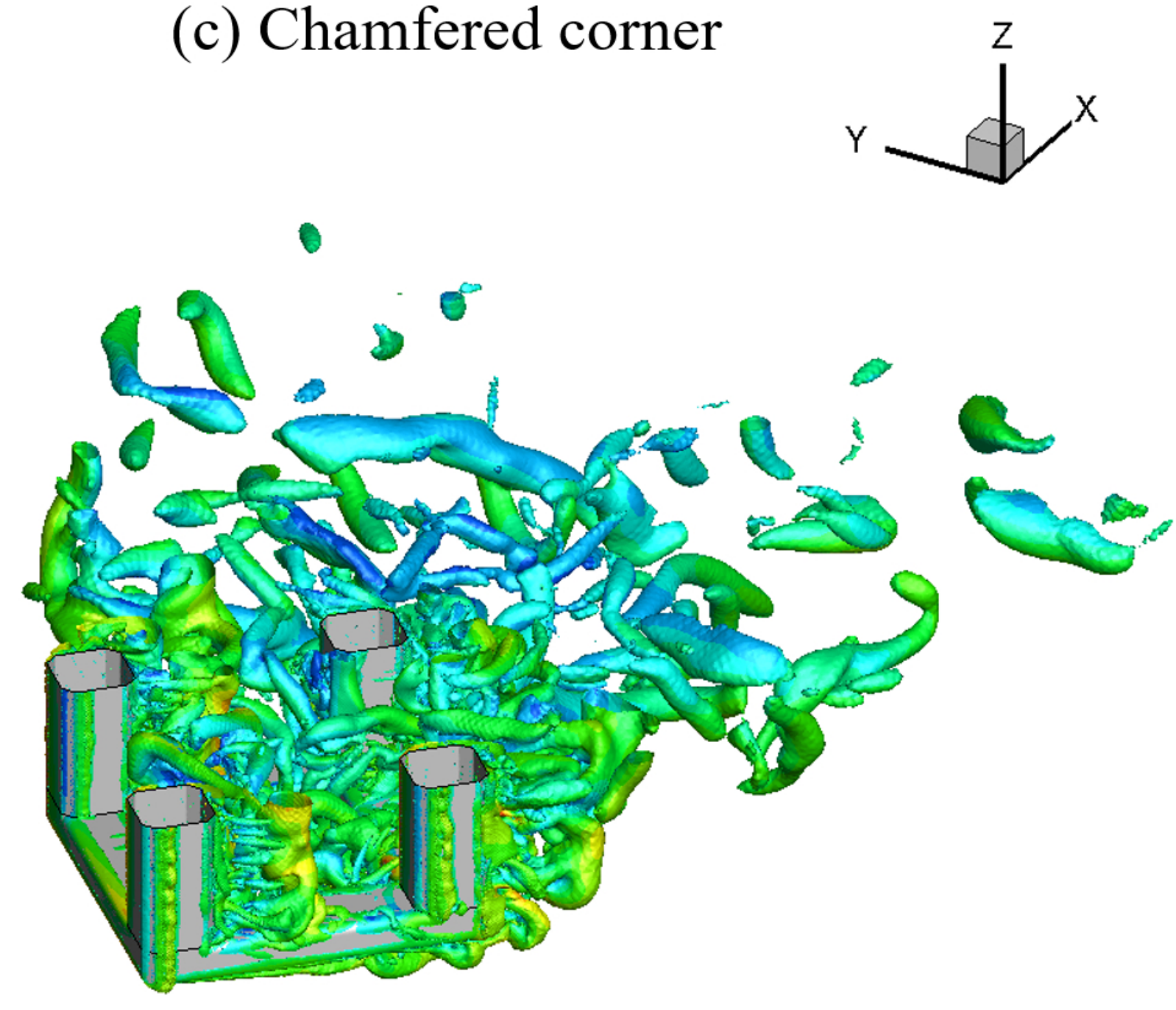

$U_{m a g} / U$ 